\title{
An updated checklist of aquatic plants of Myanmar and Thailand
}

\author{
Yu Ito ${ }^{\dagger}$, Anders S. Barfod ${ }^{\ddagger}$ \\ † University of Canterbury, Christchurch, New Zealand \\ $\ddagger$ Aarhus University, Aarhus, Denmark
}

Corresponding author: Yu Ito (yu.ito@canterbury.ac.nz)

Academic editor: Quentin Groom

Received: 04 Nov 2013 | Accepted: 29 Dec 2013 | Published: 06 Jan 2014

Citation: Ito Y, Barfod A (2014) An updated checklist of aquatic plants of Myanmar and Thailand. Biodiversity

Data Journal 2: e1019. doi: 10.3897/BDJ.2.e1019

\begin{abstract}
The flora of Tropical Asia is among the richest in the world, yet the actual diversity is estimated to be much higher than previously reported. Myanmar and Thailand are adjacent countries that together occupy more than the half the area of continental Tropical Asia. This geographic area is diverse ecologically, ranging from cool-temperate to tropical climates, and includes from coast, rainforests and high mountain elevations. An updated checklist of aquatic plants, which includes 78 species in 44 genera from 24 families, are presented based on floristic works. This number includes seven species, that have never been listed in the previous floras and checklists. The species (excluding non-indigenous taxa) were categorized by five geographic groups with the exception of to reflect the rich diversity of the countries' floras.
\end{abstract}

\section{Keywords}

Aquatic plants, flora, Myanmar, Thailand 


\section{Introduction}

Myanmar and Thailand, adjacent countries, comprise more than half of the tropical region for continental Asia. Situated within the Indo-Myanmar (Burma) hot-spot, this area is among the richest in biodiversity in the World (Van Dijk et al. 2004), with the estimated biodiversity being much higher than has been previously reported. The area is geographically diverse, going from cool-temperate to tropical and from the coast to rainforests to high mountains (Kress et al. 2003).

The aquatic floras of Myanmar and Thailand have been separately investigated. The Flora of Thailand project was launched in the 70's, led by European and Thai herbaria and institutions. The aquatic species were treated in separated volumes (Table 1). The aquatic flora of Myanmar has not been published yet, although many of the species investigated are listed in the aquatic flora of India (Cook 1996).

Table 1.

Aquatic plant families treated in volumes of Flora of Thailand.

\begin{tabular}{|c|c|c|}
\hline Volume & Family & Reference \\
\hline Volume 5 part 2 & Scrophulariaceae & Yamazaki (1990) \\
\hline Volume 7 part 2 & Callitrichaceae & Larsen (2000) \\
\hline Volume 7 part 3 & Alismataceae & Haynes (2001d) \\
\hline Volume 7 part 3 & Aponogetonaceae & Haynes (2001a) \\
\hline Volume 7 part 3 & Cymodoceaceae & Haynes (2001e) \\
\hline Volume 7 part 3 & Hydrocharitaceae & Haynes (2001b) \\
\hline Volume 7 part 3 & Lemnaceae & Landolt (2001) \\
\hline Volume 7 part 3 & Limnocharitaceae & Haynes (2001c) \\
\hline Volume 7 part 3 & Potamogetonaceae & Haynes (2001f) \\
\hline Volume 9 part 1 & Pontederiaceae & Chayamarit (2005) \\
\hline Volume 9 part 2 & Typhaceae & Simpson (2008) \\
\hline Volume 11 part 2 & Acoraceae & Boyce (2012) \\
\hline Volume 11 part 2 & Araceae & Boyce et al. (2012) \\
\hline Volume 12 part 2 & Ruppiaceae & Ito (2013a) \\
\hline
\end{tabular}




\section{Materials and methods}

The aquatic plant specimens treated in this study come from field works carried out in 2006 (Myanmar), 2008 (Myanmar), and 2012 (Thailand). Plants were hand-collected from aquatic environments e.g. ponds and lakes. Occasionally collections were made by boat (Fig. 1). In addition to field collections, exhaustive herbarium surveys were conducted in major herbaria, including AAU (Denmark), BFK (Thailand), GH (USA), MBK (Japan), TI (Japan), and TNS (Japan). The herbarium specimens were morphologically identified by using Cook (1996) or the Flora of Thailand series (Table 1). For Potamogetonaceae, in addition to the above mentioned literature, Flora of China treatment was also referred (Guo et al. 2010); for Menyanthaceae, for which no volumes for the family has been published in the Flora of Thailand, Cook (1996), Ho and Ornduff (1995), and Tippery et al. (2009) were used for identification. The identified species were classified and ordered following Haston et al. (2009). Some specimens of species cited in previous floras were examined. Species distribution was obtained from regional floras. Where distribution data were absent or where the floras have not yet been published, question marks have been inserted, e.g., ? Myanmar.

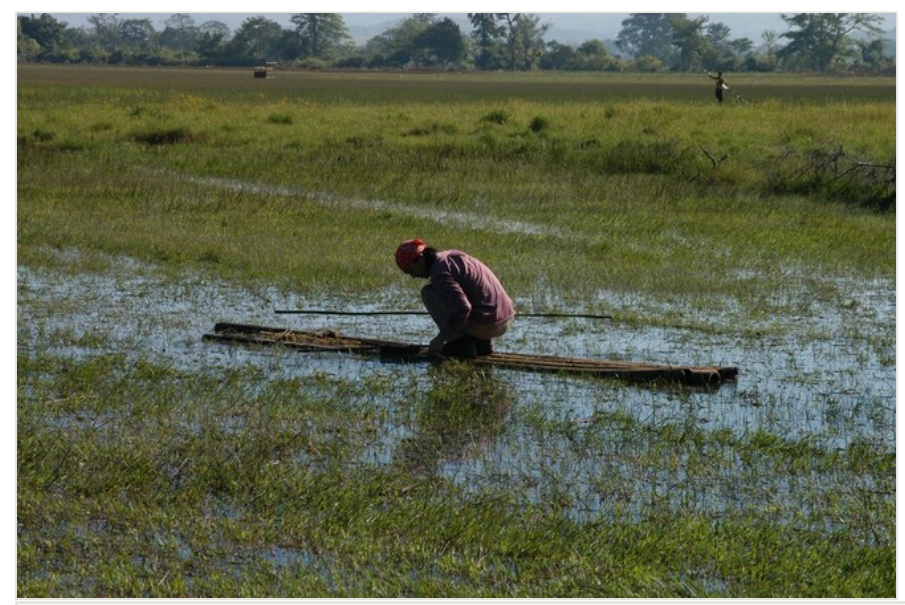

Figure 1.

Collecting aquatic plants in a lake (Myanmar). 


\section{A checklist of aquatic plants of Myanmar and Thailand (and adjoining area) \\ Order Nymphaeales}

Family Cabombaceae

Genus Cabomba Aubl., 1775

Cabomba caroliniana A. Gray., 1837

Distribution: Native to the Americas.

\section{Family Nymphaeaceae}

Genus Barclaya Wall., 1827

Barclaya longifolia Wall., 1827

Materials

a. country: Thailand; locality: Khao San Yot Natl Park.; verbatimLatitude: $12^{\circ} 9^{\prime} 15^{\prime \prime} \mathrm{N}$; verbatimLongitude: $99^{\circ}$ 58' 44" E; eventDate: Nov. 13, 2012; recordedBy: Y. Ito; collectionID: Y. Ito 1706; institutionCode: BKF

b. country: Thailand; locality: Phang Nga Province; Kuraburi District, Bangwan stream; verbatimLatitude: $9^{\circ} 13^{\prime} 34 " \mathrm{~N}$; verbatimLongitude: $98^{\circ} 26^{\prime} 22 " \mathrm{E}$; eventDate: Oct. 25 , 2006; recordedBy: Y. Ito; collectionID: T. Muadsud 137; institutionCode: BKF

c. country: Thailand; locality: Nong Khai Province; Bungkhla District, Phu Wua Wilflife Sanctuary; verbatimLatitude: $18^{\circ} 14^{\prime} 41^{\prime \prime} \mathrm{N}$; verbatimLongitude: $103^{\circ} 57^{\prime} 58^{\prime \prime} \mathrm{E}$; eventDate: Aug. 27, 2001; recordedBy: Y. Ito; collectionID: R. Pooma et al. 2781; institutionCode: BKF

d. country: Thailand; locality: Kanchanaburi Province; Sangkla Buri, Nong Lu, Tham kaeo Sawan Bandan Temple; verbatimLatitude: $15^{\circ} 16^{\prime} 20^{\prime \prime} \mathrm{N}$; verbatimLongitude: $98^{\circ} 28^{\prime} 3^{\prime \prime} \mathrm{E}$; eventDate: Aug. 25, 2010; recordedBy: Y. Ito; collectionID: V. Chamchumroon et al. 4807; institutionCode: BKF

e. country: Thailand; locality: Nong khai Province; Bungklaa, Phutoknoi; verbatimLatitude: $17^{\circ} 56^{\prime} 32$ " N; verbatimLongitude: $102^{\circ} 44^{\prime}$ 57" E; eventDate: Jun. 21, 1997; recordedBy: Y. Ito; collectionID: C. Niyomdham 5090; institutionCode: AAU

f. country: Thailand; locality: Ubon Ratchathani Province; Soi Sawan waterfall; verbatimLatitude: $15^{\circ} 27^{\prime} 35^{\prime \prime} \mathrm{N}$; verbatimLongitude: $105^{\circ} 34^{\prime} 44^{\prime \prime} \mathrm{E}$; eventDate: Nov. 15 , 2003; recordedBy: Y. Ito; collectionID: W. La-ongsri 24269; institutionCode: BKF

g. country: Thailand; locality: Ubon Ratchatani Province; Khong Chian District, Udob ratehatani Gene conservation station; verbatimLatitude: $15^{\circ} 27^{\prime} 1$ " N; verbatimLongitude: 105² 29' 2" E; eventDate: Sep. 15, 2001; recordedBy: Y. Ito; collectionID: J.F. Maxwell 01-436; institutionCode: $\mathrm{GH}$ 
h. country: Thailand; locality: Nong Khai Province; Phn Wun Wildlife Sanctuary, Thamtonn fall; verbatimLatitude: $17^{\circ} 53^{\prime} 7 " \mathrm{~N}$; verbatimLongitude: $102^{\circ} 45^{\prime} 2^{\prime \prime} \mathrm{E}$; eventDate: Jun. 14 , 2004; recordedBy: Y. Ito; collectionID: Th. Wongprasert et al. 046-85; institutionCode: BKF

i. country: Thailand; locality: Saraburi Province; Salum Lake; verbatimLatitude: $14^{\circ} 32^{\prime} \mathrm{N}$; verbatimLongitude: $101^{\circ} 2^{\prime} \mathrm{E}$; eventDate: Oct. 14, 1973; recordedBy: Y. Ito; collectionID: J.F. Maxwell 73-503; institutionCode: AAU

j. country: Thailand; locality: Chantaburi Province; $S$ of Mekam rubber plantation; verbatimLatitude: $12^{\circ} 35^{\prime} \mathrm{N}$; verbatimLongitude: $102^{\circ} 20^{\prime} \mathrm{E}$; eventDate: Aug. 22, 1966; recordedBy: Y. Ito; collectionID: K. Larsen et al. 1746; institutionCode: AAU

k. country: Thailand; locality: Phangnga Province; Takuapah District, 15 km N of Takuapah; verbatimLatitude: $8^{\circ} 53^{\prime} \mathrm{N}$; verbatimLongitude: $98^{\circ} 21^{\prime} \mathrm{E}$; eventDate: Jul. 13, 1972; recordedBy: Y. Ito; collectionID: K. Larsen et al. 30887; institutionCode: AAU

Distribution: India (Southern [Andaman IsI.]), ?Myanmar, Thailand.

Notes: Fig. 2.

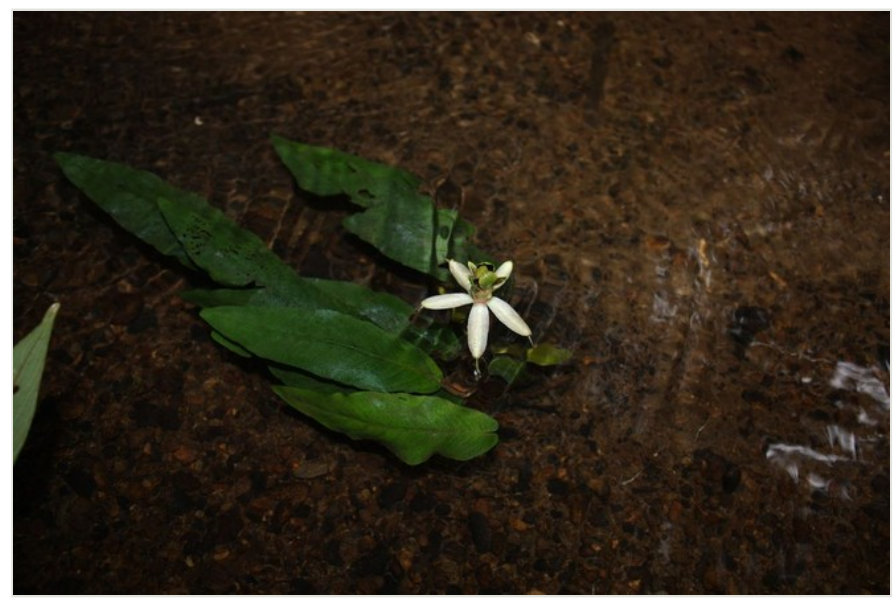

Figure 2.

Barclaya longifolia in Thailand (25 Aug. 2010). Credit: R. Pooma.

\section{Genus Nymphaea L., 1753}

\section{Nymphaea nouchali Burm. f., 1768}

\section{Materials}

a. country: Myanmar; locality: Bago Division; border to Rakhain State; verbatimLatitude: $18^{\circ}$ 40' 27" N; verbatimLongitude: $94^{\circ} 53^{\prime} 41^{\prime \prime}$ E; eventDate: Dec. 11, 2006; recordedBy: Y. Ito; collectionID: Sugawara et al. 036514; institutionCode: TI

b. country: Myanmar; locality: Rakhain State; between God village and mangrove forest; verbatimLatitude: $18^{\circ} 29^{\prime} 30^{\prime \prime} \mathrm{N}$; verbatimLongitude: $94^{\circ} 16^{\prime} 13^{\prime \prime} \mathrm{E}$; eventDate: Dec. 12 , 2006; recordedBy: Y. Ito; collectionID: Sugawara et al. 036535; institutionCode: TI 
c. country: Thailand; locality: Sattalip, Taong Breng; verbatimLatitude: $12^{\circ} 43^{\prime} \mathrm{N}$; verbatimLongitude: $100^{\circ} 56^{\prime} \mathrm{E}$; eventDate: Oct. 1, 1969; recordedBy: Y. Ito; collectionID: J.F. Maxwell s.n.; institutionCode: AAU

d. country: Thailand; locality: Narathiwat Province; S of Naratiwat; verbatimLatitude: $6^{\circ} 30^{\prime}$ $\mathrm{N}$; verbatimLongitude: $101^{\circ} 45^{\prime} \mathrm{E}$; eventDate: Mar. 8, 1974; recordedBy: Y. Ito; collectionID: K. Larsen \& S.S. Larsen 33086; institutionCode: AAU

e. country: Thailand; locality: Ayuthaya Province; Ayuthaya District; verbatimLatitude: $14^{\circ}$ 22 ' N; verbatimLongitude: $100^{\circ} 35^{\prime} \mathrm{E}$; eventDate: Dec. 3, 1979; recordedBy: Y. Ito; collectionID: T. Shimizu et al. T-26093; institutionCode: AAU

f. country: Thailand; locality: Phetchabury; verbatimLatitude: $13^{\circ} 30^{\prime} 06^{\prime \prime} \mathrm{N}$; verbatimLongitude: $99^{\circ} 47^{\prime} 37^{\prime \prime}$ E; eventDate: Nov. 14, 2012; recordedBy: Y. Ito; collectionID: Y. Ito 1711; institutionCode: BKF

Distribution: Bangladesh, China (Southern), India (nationwide), ?Indonesia, Myanmar, Pakistan, Papua New guinea, ?Philippines, Thailand, Sri Lanka, ?Vietnam.

\section{Nymphaea pubescens Willd., 1799}

\section{Materials}

a. country: Myanmar; locality: Inle lake; verbatimLatitude: $20^{\circ} 35^{\prime} 21^{\prime \prime} \mathrm{N}$; verbatimLongitude: 96 54' 34" E; eventDate: May. 1, 1938; recordedBy: Y. Ito; collectionID: F. G. Dickason 7867; institutionCode: $\mathrm{GH}$

b. country: Myanmar; locality: Bago Division; W of Pyay; verbatimLatitude: $18^{\circ} 42^{\prime} 22^{\prime \prime} \mathrm{N}$; verbatimLongitude: $95^{\circ}$ 5' 59" E; eventDate: Dec. 9, 2006; recordedBy: Y. Ito;

collectionID: Sugawara et al. 036431; institutionCode: TI

c. country: Thailand; locality: Rachaburi Province; Huai Yang waterfall; verbatimLatitude: $12^{\circ} 29^{\prime} \mathrm{N}$; verbatimLongitude: $99^{\circ} 41^{\prime} \mathrm{E}$; eventDate: Aug. 14, 1966; recordedBy: Y. Ito; collectionID: K. Larsen et al. 1588; institutionCode: AAU

d. country: Thailand; locality: Mae Sariang Province; Ban Huai Sai $12 \mathrm{~km}$ A of Mas Sariang; verbatimLatitude: $18^{\circ} 6^{\prime} \mathrm{N}$; verbatimLongitude: $97^{\circ} 55^{\prime} \mathrm{E}$; eventDate: Jul. 12, 1968; recordedBy: Y. Ito; collectionID: K. Larsen et al. 2370; institutionCode: AAU

e. country: Thailand; locality: Phattalung Province; Khuankhanum District; Talenoi Lake, Talenoi Wildlife Sanctuary; verbatimLatitude: $7^{\circ} 46^{\prime} \mathrm{N}$; verbatimLongitude: $100^{\circ} 7^{\prime} \mathrm{E}$; eventDate: Dec. 20, 1979; recordedBy: Y. Ito; collectionID: T. Shimizu et al. 27730; institutionCode: AAU

f. country: Thailand; locality: Khao San Yot Natl Park.; verbatimLatitude: $12^{\circ} 14^{\prime} 42^{\prime \prime} \mathrm{N}$; verbatimLongitude: $99^{\circ} 55^{\prime}$ 60" E; eventDate: Nov. 13, 2012; recordedBy: Y. Ito; collectionID: Y. Ito 1704; institutionCode: BKF

Distribution: Bangladesh, India (nationwide), Myanmar, Pakistan, Papua New Guinea, Thailand, Sri Lanka. 


\section{Order Acorales}

\section{Family Acoraceae}

\section{Genus Acorus L., 1753}

\section{Acorus calamus L., 1753}

\section{Materials}

a. country: Myanmar; verbatimLatitude: $16^{\circ} 53^{\prime} 19^{\prime \prime} \mathrm{N}$; verbatimLongitude: $95^{\circ} 52^{\prime} 29^{\prime \prime} \mathrm{E}$; recordedBy: Y. Ito; collectionID: MBK025720; institutionCode: TI

b. country: Myanmar; verbatimLatitude: $16^{\circ} 53^{\prime} 19.18^{\prime \prime}$; verbatimLongitude: $95^{\circ} 52^{\prime} 28.59 "$; recordedBy: Y. Ito; collectionID: MBK032409; institutionCode: TI

C. country: Myanmar; verbatimLatitude: $16^{\circ} 53^{\prime} 19.18^{\prime \prime}$; verbatimLongitude: $95^{\circ} 52^{\prime} 28.59^{\prime \prime}$; recordedBy: Y. Ito; collectionID: TI032666; institutionCode: TI

d. country: Thailand; locality: Chiang Mai Province; Juuigalion - canal, S of Chiang Mai; verbatimLatitude: $20^{\circ} 11^{\prime} \mathrm{N}$; verbatimLongitude: $99^{\circ} 46^{\prime} \mathrm{E}$; eventDate: Mar. 2, 1958; recordedBy: Y. Ito; collectionID: T. Sorensen et al. 1812; institutionCode: GH

Distribution: Worldwide.

\section{Acorus gramineus Sol. ex Aiton, 1789}

\section{Materials}

a. country: Myanmar; locality: NE Burma, Kambaiti (73 km E of Myitkyina); verbatimLatitude: $25^{\circ} 24^{\prime} 22^{\prime \prime} \mathrm{N}$; verbatimLongitude: $98^{\circ} 8^{\prime} 39^{\prime \prime} \mathrm{E}$; eventDate: Apr. 17, 1905; recordedBy: Y. Ito; collectionID: R. Malaise 85; institutionCode: $\mathrm{GH}$

b. country: Myanmar; locality: North triangle (Hbinlum) 3000'; verbatimLatitude: $20^{\circ} 21^{\prime} \mathrm{N}$; verbatimLongitude: $100^{\circ}$ 4' E; eventDate: Apr. 30, 1953; recordedBy: Y. Ito; collectionID: F. Kingdon-Ward 20756; institutionCode: GH

C. country: Myanmar; locality: Kachin division, Sumprabum Sub-Division, eastern approaches from Sumprabum to Kumon range.; verbatimLatitude: $26^{\circ} 40^{\prime} \mathrm{N}$; verbatimLongitude: $97^{\circ} 20^{\prime} \mathrm{E}$; eventDate: Mar. 1, 1962; recordedBy: Y. Ito; collectionID: J. Keenan, U. Tun Aung, U. Tha Hla 3815; institutionCode: GH

d. country: Thailand; locality: Chiang Mai Province; Muung District, Doi Sutap-Pui National Park, E side; verbatimLatitude: $18^{\circ} 48^{\prime} 16^{\prime \prime} \mathrm{N}$; verbatimLongitude: $98^{\circ} 54^{\prime} 56 " \mathrm{E}$; eventDate: Apr. 18, 1990; recordedBy: Y. Ito; collectionID: J.F. Maxwell 90-429; institutionCode: $\mathrm{GH}$

e. country: Thailand; locality: Chiang Mai Province; Doi Sutap-Pui National Park, CXU observation area; verbatimLatitude: $18^{\circ} 48^{\prime} 16^{\prime \prime} \mathrm{N}$; verbatimLongitude: $98^{\circ} 54^{\prime} 56^{\prime \prime} \mathrm{E}$; eventDate: Jan. 7, 1993; recordedBy: Y. Ito; collectionID: J.F. Maxwell 93-008; institutionCode: $\mathrm{GH}$

f. country: Thailand; locality: Lampoon Province; Mae Tah District, Doi Kuhn Dehn National Park, stream above Pah Dtoop Fall; verbatimLatitude: $18^{\circ} 30^{\prime} 25^{\prime \prime} \mathrm{N}$; verbatimLongitude: 99 16' 11" E; eventDate: Apr. 30, 1994; recordedBy: Y. Ito; collectionID: J.F. Maxwell 94-547; institutionCode: $\mathrm{GH}$ 
g. country: Thailand; locality: Lampang Province; Muang Bahn District, Jae Sawn National Park, Bah Wiang village area; verbatimLatitude: $19^{\circ} 9^{\prime} 39^{\prime \prime} \mathrm{N}$; verbatimLongitude: $99^{\circ} 23^{\prime}$ 45" E; eventDate: Feb. 15, 1996; recordedBy: Y. Ito; collectionID: J.F. Maxwell 96-227; institutionCode: $\mathrm{GH}$

h. country: Thailand; locality: Chiang Mai Province; Wiong Bah bao District, Kuhn Jae (Chae) national park; verbatimLatitude: $19^{\circ} 10^{\prime} 4 " \mathrm{~N}$; verbatimLongitude: $99^{\circ} 23^{\prime} 40^{\prime \prime} \mathrm{E}$; eventDate: Apr. 2, 1998; recordedBy: Y. Ito; collectionID: J.F. Maxwell 98-362; institutionCode: $\mathrm{GH}$

Distribution: ?Cambodia, India (north), Japan, Korea, ?Laos, Myanmar, ?Philippines, Thailand, ?Vietnam.

\section{Family Araceae}

\section{Subfamily Aroideae}

Genus Cryptocoryne Fisch. ex Wydl., 1830

\section{Cryptocoryne albida Prain, 1900}

\section{Materials}

a. country: Thailand; locality: Phang Nga province; Sri Phang Nga Natl. Park; verbatimLatitude: $8^{\circ} 0^{\prime} \mathrm{N}$; verbatimLongitude: $98^{\circ} 28^{\prime} \mathrm{E}$; eventDate: Dec. 11, 2003; recordedBy: Y. Ito; collectionID: A.S. Barfod et al. 557; institutionCode: AAU

b. country: Thailand; locality: Ranawng; verbatimLatitude: $10^{\circ} 7^{\prime} \mathrm{N}$; verbatimLongitude: $98^{\circ}$ 52' E; eventDate: Jan. 30, 1958; recordedBy: Y. Ito; collectionID: N/A 855; institutionCode: $\mathrm{GH}$

C. country: Thailand; locality: Phetchaburi, Amphoe kaeng Krachan, National Park.; verbatimLatitude: $12^{\circ} 51^{\prime} \mathrm{N}$; verbatimLongitude: $99^{\circ} 18^{\prime} \mathrm{E}$; eventDate: Dec. 12, 2002; recordedBy: Y. Ito; collectionID: D. J. Middleton et al. 1589; institutionCode: $\mathrm{GH}$

d. country: Thailand; locality: Surat Thani, phanom, Khao Sok National Park.; verbatimLatitude: $8^{\circ} 55^{\prime} \mathrm{N}$; verbatimLongitude: $98^{\circ} 31^{\prime} \mathrm{E}$; eventDate: Jan. 1,2006 ; recordedBy: Y. Ito; collectionID: D. J. Middleton et al. 3992; institutionCode: GH

Distribution: ?Myanmar, Thailand.

\section{Cryptocoryne cordata Griff., 1850}

\section{Materials}

a. country: Thailand; locality: Narathiwat Province; Paa Ye, Su Ngi Paadee; verbatimLatitude: $6^{\circ} 8^{\prime} 11^{\prime \prime} \mathrm{N}$; verbatimLongitude: $101^{\circ} 54^{\prime} 38^{\prime \prime} \mathrm{E}$; eventDate: Apr. 14 , 1988; recordedBy: Y. Ito; collectionID: C. Niyomdham \& W. Ueachirakan 1820; institutionCode: AAU 
b. country: Thailand; locality: Narathiwat Province; Freshwater swamp-forest $\mathrm{S}$ of Narathiwat; verbatimLatitude: $6^{\circ} 30^{\prime} \mathrm{N}$; verbatimLongitude: $101^{\circ} 45^{\prime} \mathrm{E}$; eventDate: Mar. 8, 1974; recordedBy: Y. Ito; collectionID: K. Larsen \& S.S. Larsen 33077; institutionCode: AAU

Distribution: Malaysia (Peninsular), Thailand.

\section{Cryptocoryne crispatula Engl., 1920}

\section{Materials}

a. country: Thailand; locality: Kanchanaburi Province; Southwest District, Pompee village ner Khwae Noi river, E of Sanghkla; verbatimLatitude: $14^{\circ} 1^{\prime} 14^{\prime \prime} \mathrm{N}$; verbatimLongitude: 99 19' 55" E; eventDate: Mar. 25, 1968; recordedBy: Y. Ito; collectionID: C.F. van Beusekom, C. Phengkhlai 89; institutionCode: AAU

b. country: Thailand; locality: Mae Hong Song, Muang District, Zuza Waterfalls, Lum Nam Pai Wildlife Sanctuary; verbatimLatitude: $19^{\circ} 28^{\prime} 29^{\prime \prime} \mathrm{N}$; verbatimLongitude: $98^{\circ} 7^{\prime} 36$ " E; eventDate: Dec. 15, 2007; recordedBy: Y. Ito; collectionID: HN8405; institutionCode: TI

c. country: Thailand; locality: Mae Hong Song, Muang District, Pha Waterfalls, Tham PlaNamtok Pha Sua Natl Park.; verbatimLatitude: $19^{\circ} 28^{\prime} \mathrm{N}$; verbatimLongitude: $98^{\circ} 7^{\prime} \mathrm{E}$; eventDate: Dec. 15, 2007; recordedBy: Y. Ito; collectionID: HN8436; institutionCode: TI

d. country: Myanmar; locality: Kachin State; between Khalone Village and Shinbwiyang; verbatimLatitude: $26^{\circ} 56^{\prime} \mathrm{N}$; verbatimLongitude: $96^{\circ} 52^{\prime} \mathrm{E}$; eventDate: Dec. 5, 2005; recordedBy: Y. Ito; collectionID: Murata et al. 041626; institutionCode: TI

e. country: Myanmar; locality: Kachin State; between Khalone Village and Shinbwiyang; verbatimLatitude: $26^{\circ} 40^{\prime} 29^{\prime \prime} \mathrm{N}$; verbatimLongitude: $96^{\circ} 16^{\prime} 33^{\prime \prime} \mathrm{E}$; eventDate: Dec. 5, 2005; recordedBy: Y. Ito; collectionID: Murata et al. 040869; institutionCode: TI

Distribution: Bangradesh, Cambodia, China, Laos, Myanmar, Thailand, Vietnam.

\section{Cryptocoryne cruddasiana Prain, 1900}

\section{Materials}

a. country: Myanmar; locality: S. ? G. Gumfrabum 1000' - 1500'; eventDate: Dec. 19, 1953; recordedBy: Y. Ito; collectionID: F. Kingdon Ward 21712; institutionCode: GH

b. country: Myanmar; locality: Kachin State; between Khalone Village and Shinbwiyang; verbatimLatitude: $26^{\circ} 40^{\prime} 50^{\prime \prime} \mathrm{N}$; verbatimLongitude: $96^{\circ} 15^{\prime} 20^{\prime \prime} \mathrm{E}$; eventDate: Dec. 5 , 2005; recordedBy: Y. Ito; collectionID: Murata et al. 041201; institutionCode: TI

Distribution: Myanmar, ?Thailand.

\section{Genus Pistia L., 1753}

\section{Pistia stratiotes L., 1753}

\section{Material}

a. country: Thailand; verbatimLocality: Bangkok; eventDate: Jul. 16, 1966; recordedBy: Y. Ito; collectionID: K. Larsen 446; institutionCode: AAU 
Distribution: Worldwide (mainly tropics).

\section{Subfamily Lemnoidea}

Genus Landoltia Les \& D.J. Crawford, 1999

Landoltia punctata (G. Mey.) Les \& D.J. Crawford, 1999

Material

a. country: Myanmar; eventDate: Dec. 3, 2008; recordedBy: Y. Ito; institutionCode: TI; collectionCode: Tanaka et al. 080648

Distribution: Worldwide.

Genus Lemna L., 1753

Lemna aequinoctialis Welw., 1859

Material

a. country: Vietnam; locality: Kontum Province; Ngoc Linh mountain, Ngoc Linh village.; verbatimLatitude: $14^{\circ} 20^{\prime} \mathrm{N}$; verbatimLongitude: $107^{\circ} 59^{\prime} \mathrm{E}$; eventDate: Apr. 4, 1995; recordedBy: Y. Ito; collectionID: L. Averyanov et al. VH1199; institutionCode: AAU

Distribution: Bangladesh, China (nationwide), India (nationwide), Japan, Myanmar, Nepal, Pakistan, Thailand; Africa; Oceania; N. America; S. America.

\section{Lemna trisulca L., 1753}

Material

a. country: Myanmar; locality: Shan State; Inlay lake, Nyanug She Township; verbatimLatitude: $20^{\circ} 32^{\prime}$ 2" N; verbatimLongitude: $96^{\circ} 53^{\prime} 53^{\prime \prime}$ E; eventDate: Dec. 3 , 2008; recordedBy: Y. Ito; collectionID: Tanaka et al. 080649; institutionCode: TI

Distribution: Bangladesh, China (Northern, Western, Southern [Taiwan, Yunnan]), India (Eastern, Northern), Indonesia (New Guinea), Japan, Malaysia (Sumatra), Myanmar, Papua New guinea, Pakistan, Philippines; Europe; Oceania; N. America; S. America. 
Genus Spirodela Schleid., 1839

Spirodela polyrrhiza (L.) Schleid., 1839

Material

a. country: Myanmar; locality: Shan State; Inlay lake, Nyanug She Township; verbatimLatitude: $20^{\circ} 32^{\prime} 2^{\prime \prime} \mathrm{N}$; verbatimLongitude: $96^{\circ} 53^{\prime} 53^{\prime \prime} \mathrm{E}$; eventDate: Dec. 3 , 2008; recordedBy: Y. Ito; collectionID: Tanaka et al. 080648; institutionCode: MBK

Distribution: Bangladesh, China (nationwide), India (nationwide), Indonesia (Java, Sumatra, Sulawesi), Japan, Malaysia (Peninsular), Myanmar, Nepal, Pakistan, Papua New Guinea, Philippines, Thailand.

\section{Genus Wolffia Horkel ex Schleid., 1844}

\section{Wolffia globosa (Roxb.) Hartog \& Plas, 1970}

Distribution: Bangladesh, ?Cambodia, India (nationwide), ?Indonesia, Japan, ?Laos, ?Malaysia, ?Myanmar, Nepal, Pakistan, Papua New Guinea, ?Philippines, Sri Lanka, Thailand, ?Vietnam; introduced in Americas.

\section{Order Alismatales}

\section{Family Alismataceae}

Genus Alisma L., 1753

\section{Alisma plantago-aquatica L., 1753}

Materials

a. country: Myanmar; locality: Mandalay Division; Pyin U Lwin (Maymyo); eventDate: May 1, 1932; recordedBy: Y. Ito; collectionID: F. G. Dickason 5798; institutionCode: GH

b. country: Myanmar; locality: Shan State, Kalow; eventDate: Nov. 26, 2008; recordedBy: Y. Ito; collectionID: Tanaka et al. 080047; institutionCode: TI

c. country: Myanmar; locality: Shan State; Kalow; eventDate: May 1, 1932; recordedBy: Y. Ito; collectionID: F. G. Dickason 5743; institutionCode: GH

d. country: Myanmar; locality: Shan State; Kalow; eventDate: May 8, 1931; recordedBy: Y. Ito; collectionID: F. G. Dickason; institutionCode: GH

e. country: Thailand; locality: Chiang Mai; above Bong Lom Tang (Karen) village, mae Win Subdistrict; eventDate: Mar. 21, 2003; recordedBy: Y. Ito; collectionID: J.F. Maxwell 03-55; institutionCode: GH

f. country: Thailand; locality: Chiang Mai Province; between Ban Bo Luang and Mae Sariang; eventDate: Jun. 13, 1968; recordedBy: Y. Ito; collectionID: C.F. van Beusekom, C. Phengkhlai 1200; institutionCode: AAU 
g. country: Thailand; locality: Chiang Mai Province; Hod District, Mai Muang Nao Arboretum, Ban Mae Sanam Mai, Baw Salee Subdistrict, 53 km Hod - Mae Sariang road (hw. 108); eventDate: Apr. 11, 2001; recordedBy: Y. Ito; collectionID: W. Sankamethawee 151; institutionCode: BKF

h. country: Thailand; locality: Mae Hong Son Province; Mae Sariung, Ban Papae; verbatimLatitude: $18^{\circ} 54^{\prime} \mathrm{N}$; verbatimLongitude: $98^{\circ} 8^{\prime} \mathrm{E}$; eventDate: Feb 2, 1969 ; recordedBy: Y. Ito; collectionID: T. Leiitinand e Sa-nga 10671; institutionCode: AAU

Distribution: China (North-Eastern, North-Western, South [Yunnan]), Japan, Myanmar, Nepal, Thailand; Europe; Oceania; N. America.

\section{Genus Caldesia Parl., 1860}

\section{Caldesia parnassifolia (Bassi ex L.) Parl., 1858}

\section{Materials}

a. country: Laos; locality: Savannaket Province; Nakai Plateau, Theun Douan lake, near Phong Sa Vahn resettlement village.; verbatimLatitude: $16^{\circ} 34^{\prime} 10^{\prime \prime} \mathrm{N}$; verbatimLongitude: $104^{\circ} 44^{\prime}$ 54" E; eventDate: May 3, 2007; recordedBy: Y. Ito; collectionID: J.F. Maxwell 07-305; institutionCode: $\mathrm{GH}$

b. country: Myanmar; locality: Shan State; Taunggyi; verbatimLatitude: $20^{\circ} 47^{\prime} 7^{\prime \prime} \mathrm{N}$; verbatimLongitude: $97^{\circ} 2^{\prime}$ 6" E; eventDate: May 1, 1938; recordedBy: Y. Ito; collectionID: F. G. Dickason 9370; institutionCode: GH

C. country: Myanmar; locality: Shan State, Pindaya; verbatimLatitude: $20^{\circ} 56^{\prime} 21^{\prime \prime} \mathrm{N}$; verbatimLongitude: $96^{\circ} 39^{\prime}$ 54" E; eventDate: Dec. 1, 2008; recordedBy: Y. Ito; collectionID: Tanaka et al. 080624 \#1; institutionCode: TI

d. country: Myanmar; locality: Shan State, Pindaya; verbatimLatitude: $20^{\circ} 56^{\prime} 21^{\prime \prime}$; verbatimLongitude: $96^{\circ} 39^{\prime} 54^{\prime \prime} \mathrm{E}$; eventDate: Dec. 1, 2008; recordedBy: Y. Ito; collectionID: Tanaka et al. 080624 \#2; institutionCode: TI

Distribution: Bangladesh, China (North-Eastern, South [Yunnan]), Japan, India (North, South, West), Indonesia (Sulawesi, New Guinea), Laos, Myanmar, Nepal, Papua New guinea, Thailand, ?Vietnam; Africa; Europe; Oceania.

Notes: Myanmar (Ito et al. 2009).

\section{Genus Limnocharis Bonpl., 1808}

\section{Limnocharis flava (L.) Buchenau, 1868}

Materials

a. country: Myanmar; locality: Rangoon.; verbatimLatitude: $16^{\circ} 49^{\prime} 37^{\prime \prime} \mathrm{N}$ verbatimLongitude: $96^{\circ} 8^{\prime}$ 58" E; eventDate: Mar. 10, 1957; recordedBy: Y. Ito; collectionID: F. G. Dickason 6666; institutionCode: GH

b. country: Myanmar; locality: Rakhine State; Bago Division; verbatimLatitude: $18^{\circ} 49^{\prime} 44^{\prime \prime}$ $\mathrm{N}$; verbatimLongitude: $95^{\circ}$ 18' 6" E; eventDate: Dec. 7, 2008; recordedBy: Y. Ito; collectionID: Tanaka et al. 080766; institutionCode: MBK 
c. country: Thailand; locality: Chiang Mai; verbatimLatitude: $19^{\circ} 23^{\prime} \mathrm{N}$; verbatimLongitude: 99 10' E; eventDate: Feb. 14, 1958; recordedBy: Y. Ito; collectionID: Kau Cloi 731; institutionCode: AAU

d. country: Thailand; locality: Sattalip, Taong Grang; verbatimLatitude: $12^{\circ} 43^{\prime} \mathrm{N}$; verbatimLongitude: $100^{\circ} 56^{\prime} \mathrm{E}$; eventDate: Jan. 24, 1969; recordedBy: Y. Ito; collectionID: J.F. Maxwell; institutionCode: AAU

e. country: Thailand; locality: Trang Province: Muang District; Kaochong Park; verbatimLatitude: $7^{\circ} 33^{\prime} 53^{\prime \prime} \mathrm{N}$; verbatimLongitude: $99^{\circ} 36^{\prime} 37^{\prime \prime} \mathrm{E}$; eventDate: Feb. 3 , 1985; recordedBy: Y. Ito; collectionID: P. Sirirugsa 990; institutionCode: AAU

f. country: Thailand; locality: Trang Province; Muang District, Kao Chong Park.; verbatimLatitude: $7^{\circ} 33^{\prime} 53^{\prime \prime} \mathrm{N}$; verbatimLongitude: 99³6' 37" E; eventDate: Oct. 31, 1984; recordedBy: Y. Ito; collectionID: P. Sirirugsa 939; institutionCode: GH

g. country: Thailand; locality: Chiang Mai Province; $30 \mathrm{~km} \mathrm{SW}$ of Chiengmai.; verbatimLatitude: $19^{\circ} 11^{\prime} \mathrm{N}$; verbatimLongitude: $98^{\circ}$ 4' E; eventDate: Nov. 4, 1958; recordedBy: Y. Ito; collectionID: No. 6043; institutionCode: GH

h. country: Thailand; locality: Petchabury; verbatimLatitude: $13^{\circ} 24^{\prime} 30^{\prime \prime} \mathrm{N}$; verbatimLongitude: $99^{\circ} 48^{\prime} 44^{\prime \prime}$ E; eventDate: Nov. 14, 2012; recordedBy: Y. Ito; collectionID: YI1710; institutionCode: BKF

Distribution: Native to Americas; naturalized to tropical Asia.

\section{Genus Sagittaria L., 1753}

\section{Sagittaria guayanensis Humb., 1815}

\section{Materials}

a. country: Thailand; locality: Pathum Thani Province, Rangsit; verbatimLatitude: $13^{\circ} 59^{\prime} 17^{\prime \prime}$ $\mathrm{N}$; verbatimLongitude: $100^{\circ} 37^{\prime} 59^{\prime \prime} \mathrm{E}$; eventDate: Aug. 25, 1974; recordedBy: Y. Ito; collectionID: S. Sripen s.n.; institutionCode: BKF

b. country: Thailand; locality: Mae Hong Son Province; verbatimLatitude: $19^{\circ} 15^{\prime} \mathrm{N}$; verbatimLongitude: $98^{\circ} \mathrm{E}$; eventDate: Sep. 9, 1974; recordedBy: Y. Ito; collectionID: K. Larsen \& S.S. Larsen 34312; institutionCode: BKF

C. country: Thailand; locality: Mae Hong Son Province; verbatimLatitude: $19^{\circ} 15^{\prime} \mathrm{N}$; verbatimLongitude: $98^{\circ}$ E; eventDate: Sep. 10, 1974; recordedBy: Y. Ito; collectionID: K. Larsen \& S.S. Larsen 34375; institutionCode: BKF

d. country: Thailand; locality: Chiang Mai; Doi Lodge; verbatimLatitude: $19^{\circ} 18^{\prime} \mathrm{N}$; verbatimLongitude: $99^{\circ} 10^{\prime} \mathrm{E}$; eventDate: Apr. 15, 1958; recordedBy: Y. Ito; collectionID: No. 2742; institutionCode: $\mathrm{GH}$

Distribution: Bangladesh, ?Cambodia, China (Central, South), India (nationwide), Indonesia (Java, Sumatra, Sulawesi), Malaysia (Peninsular), Nepal, Thailand, ? Vietnam. 


\section{Sagittaria trifolia L., 1753}

\section{Materials}

a. country: Myanmar; locality: Shan State; Taungyi; verbatimLatitude: $20^{\circ} 47^{\prime} \mathrm{N}$; verbatimLongitude: $97^{\circ} 2^{\prime} \mathrm{E}$; eventDate: May. 3, 1933; recordedBy: Y. Ito; collectionID: F. G. Dickason 5846; institutionCode: $\mathrm{GH}$

b. country: Thailand; locality: Chiang Mai; $15 \mathrm{~km} \mathrm{~N}$ of Chiang Mai; verbatimElevation: $18^{\circ}$ 55' N; verbatimLatitude: $98^{\circ}$ 57' E; eventDate: Aug. 11, 1958; recordedBy: Y. Ito; collectionID: Kai Larsen 6057; institutionCode: GH

c. country: Thailand; locality: Chiang Mai Province; Phek Khaeng Kai; verbatimLatitude: $18^{\circ}$ 47' 30" N; verbatimLongitude: 98 57' 39" E; eventDate: Feb. 20, 1991; recordedBy: Y. Ito; collectionID: R. Pooma 91033; institutionCode: GH

d. country: Thailand; locality: Chiang Mai; Mae Sanam Mai village, Baw Sahlee subdistrict; verbatimLatitude: $18^{\circ} 58^{\prime} 44^{\prime \prime} \mathrm{N}$; verbatimLongitude: $98^{\circ}$ 56' 3" E; eventDate: Jul. 2, 2000; recordedBy: Y. Ito; collectionID: J.F. Maxwell 00-307; institutionCode: GH

e. country: Thailand; locality: Chiang Mai; Mae Ria District, Doi Suthep-Pui National Park, N side; verbatimLatitude: $18^{\circ} 49^{\prime} 28^{\prime \prime} \mathrm{N}$; verbatimLongitude: $98^{\circ} 53^{\prime} 24 " \mathrm{E}$; eventDate: Feb. 20, 1990; recordedBy: Y. Ito; collectionID: J.F. Maxwell 90-216; institutionCode: GH

f. country: Myanmar; locality: Shan State; Taungyi, Namkok; eventDate: May. 1, 1938; recordedBy: Y. Ito; collectionID: F. G. Dickason 9405; institutionCode: GH

g. country: Myanmar; locality: Shan State; Inle lake; eventDate: May. 1, 1938; recordedBy: Y. Ito; collectionID: F. G. Dickason 7865; institutionCode: GH

h. country: Myanmar; locality: Shan State, Kalow; eventDate: Nov. 26, 2008; recordedBy: Y. Ito; collectionID: Tanaka et al. 080046; institutionCode: TI

i. country: Myanmar; locality: Shan State, Pindaya; verbatimLatitude: $20^{\circ} 59^{\prime} 57^{\prime \prime} \mathrm{N}$; verbatimLongitude: $96^{\circ} 39^{\prime}$ 59" E; eventDate: Dec. 1, 2008; recordedBy: Y. Ito; collectionID: Tanaka et al. 080623; institutionCode: TI

j. country: Thailand; locality: Chiang Mai; Mai Muang Nao Arboretum, Bam Mae Sanam Mai, Baw Salee Subdistrict; verbatimLatitude: $18^{\circ} 9^{\prime} 41^{\prime \prime} \mathrm{N}$; verbatimLongitude: $98^{\circ} 16^{\prime}$ 54" E; eventDate: Apr. 11, 2001; recordedBy: Y. Ito; collectionID: W. Sankamethawee 150; institutionCode: $\mathrm{GH}$

k. country: Thailand; locality: Chiang Mai Province; Hot District; Maesanaam Pine Inprovement Center; verbatimLatitude: $18^{\circ} 11^{\prime} \mathrm{N}$; verbatimLongitude: $98^{\circ} 36^{\prime} \mathrm{E}$; eventDate: Jul. 8, 1995; recordedBy: Y. Ito; collectionID: R. Pooma et al. 1032; institutionCode: BKF

I. country: Thailand; locality: Chiang Mai Province; Om-koi; eventDate: Jul. 20, 1987; recordedBy: Y. Ito; collectionID: C. Phengklai et al. 6277; institutionCode: BKF

Distribution: Bangladesh, Bhutan, China (nationwide), India (nationwide), Indonesia (Borneo, Java, Sulawesi), Japan, Malaysia (Peninsular), Myanmar, Nepal, Pakistan, Philippines, Thailand; Oceania. 


\section{Family Hydrocharitaceae}

\section{Genus Blyxa Noronha ex Thouars, 1806}

\section{Blyxa aubertii Rich., 1812}

\section{Materials}

a. country: Myanmar; verbatimLatitude: $16^{\circ} 53^{\prime} 19^{\prime \prime} \mathrm{N}$; verbatimLongitude: $95^{\circ} 52^{\prime} 29 " \mathrm{E}$; recordedBy: Y. Ito; collectionID: MBK041203; institutionCode: TI

b. country: Thailand; locality: Trat Province; $20 \mathrm{~km} \mathrm{~W}$ of Trat; verbatimLatitude: $12^{\circ} 30^{\prime} \mathrm{N}$; verbatimLongitude: $102^{\circ} 20^{\prime} \mathrm{E}$; eventDate: Aug. 30, 1972; recordedBy: Y. Ito; collectionID: K. Larsen et al. 32232; institutionCode: AAU

C. country: Thailand; locality: Trat Province; $18 \mathrm{~km} \mathrm{~W}$ of Trat; verbatimLatitude: $12^{\circ} 40^{\prime} \mathrm{N}$; verbatimLongitude: $102^{\circ} 16^{\prime} \mathrm{E}$; eventDate: Oct. 23, 1972; recordedBy: Y. Ito; collectionID: J.F. Maxwell 72-540; institutionCode: AAU

d. country: Thailand; locality: Chantaburi province; Plain of Makam; verbatimLatitude: $12^{\circ}$ 41' N; verbatimLongitude: $102^{\circ} 15^{\prime} \mathrm{E}$; eventDate: Aug. 22, 1966; recordedBy: Y. Ito; collectionID: K. Larsen et al. 1667; institutionCode: AAU

e. country: Thailand; locality: Rayong Province; Ban phe; verbatimLatitude: $12^{\circ} 40^{\prime} \mathrm{N}$; verbatimLongitude: $101^{\circ} 25^{\prime} \mathrm{E}$; eventDate: Dec. 16,1974 ; recordedBy: Y. Ito; collectionID: R. Geesik \& P. Hiepko 7872; institutionCode: AAU

f. country: Thailand; locality: Chantaburi Province; $S$ of Mekam rubber plantation; verbatimLatitude: $12^{\circ} 35^{\prime} \mathrm{N}$; verbatimLongitude: $102^{\circ} 20^{\prime} \mathrm{E}$; eventDate: Aug. 22, 1966; recordedBy: Y. Ito; collectionID: K. Larsen et al. 1744; institutionCode: AAU

g. country: Thailand; locality: Narathiwat Province; Tak bai; verbatimLatitude: $6^{\circ} 15^{\prime} 34$ " N; verbatimLongitude: $102^{\circ}$ 0' 16" E; eventDate: Jan. 11, 1986; recordedBy: Y. Ito; collectionID: C. Cniyomdham 1117; institutionCode: AAU

h. country: Thailand; locality: Phangnga Province; Takuapah District; 15 km N of Takuapah; verbatimLatitude: $8^{\circ} 53$ 'N; verbatimLongitude: $98^{\circ} 21^{\prime} \mathrm{E}$; eventDate: Jul. 14, 1972; recordedBy: Y. Ito; collectionID: K. Larsen et al. 30970; institutionCode: AAU

i. country: Vietnam; locality: Lao Cai Province; Than Uyen District, Municipality Ho Mit; verbatimLatitude: $22^{\circ} 6^{\prime} \mathrm{N}$; verbatimLongitude: $103^{\circ}$ 52' E; eventDate: May. 21, 1999; recordedBy: Y. Ito; collectionID: NTH2708; institutionCode: AAU

Distribution: Bangladesh, China (Southern), India (Western, Central, Southern), Indonesia (Java, Sumatra), Japan, Malaysia (Peninsular), Myanmar, ?Nepal, Papua New guinea, Sri Lanka, Thailand, Vietnam.

\section{Blyxa echinosperma (C.B. Clarke) Hook. f., 1888}

\section{Materials}

a. country: Myanmar; locality: Kachin State; verbatimLatitude: $26^{\circ} 40^{\prime} 50^{\prime \prime} \mathrm{N}$; verbatimLongitude: $96^{\circ} 15^{\prime} 20^{\prime \prime}$ E; eventDate: Dec. 10, 2005; recordedBy: Y. Ito; collectionID: J. Murata et al. 040940; institutionCode: TI

b. country: Thailand; locality: Phangnga Province, Khuraburi, Ko Phrathong; verbatimLatitude: $9^{\circ} 11^{\prime} 45^{\prime \prime} \mathrm{N}$; verbatimLongitude: $98^{\circ} 24^{\prime} 30^{\prime \prime} \mathrm{E}$; eventDate: Aug. 24, 2005; recordedBy: Y. Ito; collectionID: C. Phengklai et al. 15062; institutionCode: BKF 
c. country: Thailand; locality: Chiang Mai; near Angka Noi village, $\mathrm{N}$ side of the main road, Doi Inthanon.; verbatimLatitude: $18^{\circ} 35^{\prime} 32^{\prime \prime} \mathrm{N}$; verbatimLongitude: $98^{\circ} 29^{\prime} 12^{\prime \prime} \mathrm{E}$; eventDate: May. 8, 1988; recordedBy: Y. Ito; collectionID: N. Fukuoka T-62538; institutionCode: $\mathrm{TI}$

Distribution: Bangladesh, China (Central, Southern), India (Western, Central, Southern), Indonesia (Java), Japan, Malaysia (Borneo, Peninsular), Myanmar, ?Nepal, Papua New Guinea, Philippines, Thailand, Sri Lanka; Oceania.

\section{Blyxa japonica (Miq.) Maxim. ex Asch. \& Gürke, 1889}

\section{Materials}

a. country: Cambodia; locality: Kampot Province; Kampot District, S summit of Phnom Bokor, Bokor village; verbatimLatitude: $10^{\circ} 37^{\prime} 4$ " N; verbatimLongitude: $104^{\circ} 1^{\prime} 41^{\prime \prime}$ E; eventDate: Jun. 18, 1997; recordedBy: Y. Ito; collectionID: McDonald et al. 5793; institutionCode: AAU

b. country: Thailand; locality: Naratiwat Province; Sungeipadi District; verbatimLatitude: $6^{\circ}$ 29' 4" N; verbatimLongitude: $101^{\circ} 49^{\prime} 2^{2}$ " E; eventDate: Mar. 28, 1987; recordedBy: Y. Ito; collectionID: J.F. Maxwell 87-265; institutionCode: GH

c. country: Thailand; locality: E Si Saket Province; between Uthum Phon Phisai and Si Saket; verbatimLatitude: $15^{\circ} 7^{\prime} \mathrm{N}$; verbatimLongitude: $104^{\circ} 15^{\prime} \mathrm{E}$; eventDate: Oct. 7 , 1984; recordedBy: Y. Ito; collectionID: G. Murata, C. Phengklai, S. Mitsuta, H. Nagamasu, N. Nantasan T-49920; institutionCode: GH

d. country: Thailand; locality: Kanchanaburi Province; Thong Pha Phum District, Rintin Forest; verbatimLatitude: $14^{\circ} 50^{\prime} 9^{\prime \prime} \mathrm{N}$; verbatimLongitude: $98^{\circ} 44^{\prime} 21^{\prime \prime} \mathrm{E}$; eventDate: Nov. 6, 1979; recordedBy: Y. Ito; collectionID: T. Shimizu et al. T-21941; institutionCode: AAU

e. country: Thailand; locality: Trat Province; $20 \mathrm{~km} \mathrm{~W}$ of Trat; verbatimLatitude: $12^{\circ} 30^{\prime} \mathrm{N}$; verbatimLongitude: $102^{\circ} 20^{\prime} \mathrm{E}$; eventDate: Aug. 30, 1972; recordedBy: Y. Ito;

collectionID: K. Larsen et al. 32234; institutionCode: AAU

f. country: Thailand; locality: Nakhon Ratchasima Province; Khao yai Natl Park, Khao Laem; verbatimLatitude: $14^{\circ} 45^{\prime} \mathrm{N}$; verbatimLongitude: $102^{\circ} \mathrm{E}$; eventDate: Oct. 19, 1969; recordedBy: Y. Ito; collectionID: C.F. van Beusekom, C. Phengkhlai 1769; institutionCode: AAU

g. country: Thailand; locality: Narathiwat Province; Tak bai; verbatimLatitude: $6^{\circ} 14^{\prime} 57^{\prime \prime} \mathrm{N}$; verbatimLongitude: $102^{\circ}$ 0' 6" E; eventDate: Jan. 11, 1986; recordedBy: Y. Ito; collectionID: C. Cniyomdham 1116; institutionCode: AAU

h. country: Thailand; locality: Chantaburi Province; verbatimLatitude: $12^{\circ} 57^{\prime} \mathrm{N}$; verbatimLongitude: $102^{\circ} \mathrm{E}$; eventDate: Oct. 17, 1971; recordedBy: Y. Ito; collectionID: J.F. Maxwell 71-576; institutionCode: AAU

i. country: Thailand; locality: Saraburi Province; Salum Lake; verbatimLatitude: $14^{\circ} 32^{\prime} \mathrm{N}$; verbatimLongitude: $101^{\circ} 2$ ' E; eventDate: Oct. 14, 1973; recordedBy: Y. Ito; collectionID: J.F. Maxwell 73-504; institutionCode: AAU

j. country: Thailand; locality: Pha Team National Park, Ubon Ratchathani; verbatimLatitude: $15^{\circ} 24^{\prime} 10^{\prime \prime} \mathrm{N}$; verbatimLongitude: $105^{\circ} 29^{\prime} 23^{\prime \prime} \mathrm{E}$; recordedBy: Y. Ito; collectionID: Nr. Tanaka; institutionCode: TNS

Distribution: Bangladesh, China (nationwide), Cambodia, India (Western [Meghalaya]), Indonesia (Boreo, New Guinea, Sulawesi), Japan, Malaysia (Peninsular), Myanmar, Papua New Guinea, Thailand. 


\section{Blyxa quadricostata den Hartog, 1973}

\section{Materials}

\section{Other materials:}

a. country: Thailand; locality: Naratiwat Province; Sungei Padi District, Sungei Padi; verbatimLatitude: $6^{\circ} 7^{\prime} 27^{\prime \prime} \mathrm{N}$; verbatimLongitude: $101^{\circ} 54^{\prime} 47^{\prime \prime} \mathrm{E}$; eventDate: Mar. 28 , 1987; recordedBy: Y. Ito; collectionID: J.F. Maxwell 87-267; institutionCode: GH

b. country: Thailand; locality: Loei Province; Phu Kradung; verbatimLatitude: $17^{\circ} 16^{\prime} 54^{\prime \prime} \mathrm{N}$; verbatimLongitude: $101^{\circ}$ 9' 31" E; eventDate: Nov. 29, 1965; recordedBy: Y. Ito; collectionID: M. Tagawa et al. T-781; institutionCode: AAU

c. country: Thailand; locality: Loei Province; Phu Kradung; verbatimLatitude: $16^{\circ} 53^{\prime} \mathrm{N}$; verbatimLongitude: $101^{\circ} 47^{\prime} \mathrm{E}$; eventDate: Nov. 2, 1984; recordedBy: Y. Ito; collectionID: G. Murata et al. T-42713; institutionCode: AAU

d. country: Thailand; locality: Loei Province; Phu Kradung District, Phu Kradung Natl park; verbatimLatitude: $17^{\circ} 16^{\prime} 54^{\prime \prime} \mathrm{N}$; verbatimLongitude: $101^{\circ} 9^{\prime} 31$ " E; eventDate: Nov. 15 , 1979; recordedBy: Y. Ito; collectionID: T. Shimizu et al. T-22824; institutionCode: AAU

e. country: Thailand; locality: Loei Province; Phu Kradung District, Phu Kradung Natl park; verbatimLatitude: $17^{\circ} 16^{\prime} 54^{\prime \prime} \mathrm{N}$; verbatimLongitude: $101^{\circ}$ 9' 31" E; eventDate: Mar. 9 , 1979; recordedBy: Y. Ito; collectionID: P.J. O'Connor \& C. Niyondham 15711; institutionCode: AAU

f. country: Thailand; locality: Loei Province; Phu Kradung; verbatimLatitude: $17^{\circ} 17^{\prime} \mathrm{N}$; verbatimLongitude: $101^{\circ} 10^{\prime} \mathrm{E}$; eventDate: Mar. 18, 1958; recordedBy: Y. Ito; collectionID: Th. Sorensen 2211; institutionCode: AAU

g. country: Thailand; locality: Loei Province; Phu Kradung, S. of Loi; verbatimLatitude: $16^{\circ}$ 53' N; verbatimLongitude: $101^{\circ}$ 53' E; eventDate: Nov. 7, 1970; recordedBy: Y. Ito; collectionID: Ch. Charoenphol et al. 4783; institutionCode: AAU

\section{Holotype:}

a. country: Thailand; locality: Loei Province; Phu Kradung, Mie ron Pond; verbatimLatitude: $16^{\circ} 52^{\prime} \mathrm{N}$; verbatimLongitude: $101^{\circ} 52^{\prime} \mathrm{E}$; eventDate: Dec. 25 , 1971; recordedBy: Y. Ito; collectionID: C.F. van Beusekom et al. 4602; institutionCode: MO

Distribution: Thailand.

\section{Genus Egeria Planch., 1849}

\section{Egeria densa (Planch.) Casp., 1857}

\section{Material}

a. country: Thailand; locality: Bung Bonapet Nonhunting Area; verbatimLatitude: $15^{\circ} 41^{\prime} 40^{\prime \prime}$ $\mathrm{N}$; verbatimLongitude: $100^{\circ} 16^{\prime} 03^{\prime \prime} \mathrm{E}$; eventDate: Nov. 16, 2012; recordedBy: Y. Ito; collectionID: Y. Ito 1733; institutionCode: BKF

Distribution: Native to South America. 
Genus Elodea Michx., 1803

Elodea nuttallii (Planch.) H. St. John, 1920

Distribution: Native to North America.

\section{Genus Hydrilla Rich., 1814}

\section{Hydrilla verticillata (L. f.) Royle, 1839}

\section{Materials}

a. country: Myanmar; stateProvince: Kachin; verbatimLocality: Tanaing Township; verbatimLatitude: $26^{\circ} 22^{\prime} 22^{\prime \prime} \mathrm{N}$; verbatimLongitude: $96^{\circ} 43^{\prime}$ E; eventDate: Sep. 15, 2005; recordedBy: Y. Ito; collectionID: MBK040056; institutionCode: TI

b. country: Myanmar; stateProvince: Shan State; locality: Pindaya; verbatimLatitude: $20^{\circ} 59^{\prime}$ 57" N; verbatimLongitude: $96^{\circ} 39^{\prime}$ 59" E; recordedBy: Y. Ito; collectionID: MBK080634; institutionCode: $\mathrm{TI}$

c. country: Myanmar; locality: Mandalay Division; verbatimLatitude: $22^{\circ} 00^{\prime} 29^{\prime \prime} \mathrm{N}$; verbatimLongitude: $96^{\circ} 28^{\prime} 06^{\prime \prime} \mathrm{E}$; eventDate: Jan. 11, 2002; recordedBy: Y. Ito; collectionID: Tanaka et al. 021712; institutionCode: TI

d. country: Myanmar; locality: Mandalay Division; verbatimLatitude: $20^{\circ} 48^{\prime} \mathrm{N}$; verbatimLongitude: $95^{\circ} 15^{\prime} \mathrm{E}$; eventDate: Mar. 4, 2003; recordedBy: Y. Ito; collectionID: Tanaka et al. 028704; institutionCode: TI

e. country: Myanmar; locality: Bago Division; verbatimLatitude: $18^{\circ} 42^{\prime} 22^{\prime \prime} \mathrm{N}$; verbatimLongitude: $95^{\circ}$ 5' 59" E; eventDate: Dec. 9, 2006; recordedBy: Y. Ito; collectionID: Sugawara et al. 036433; institutionCode: TI

f. country: Myanmar; locality: Kachin State; verbatimLatitude: $26^{\circ} 6^{\prime} 34^{\prime \prime} \mathrm{N}$; verbatimLongitude: $96^{\circ} 42^{\prime} 58^{\prime \prime} \mathrm{E}$; eventDate: Sep. 19, 2005; recordedBy: Y. Ito; collectionID: Tanaka et al. 040483; institutionCode: TI

g. country: Myanmar; locality: Shan State; verbatimLatitude: $20^{\circ} 35^{\prime} 41^{\prime \prime} \mathrm{N}$; verbatimLongitude: $96^{\circ} 31^{\prime} 46^{\prime \prime} \mathrm{E}$; eventDate: Nov. 26, 2008; recordedBy: Y. Ito; collectionID: Tanaka et al. 080050; institutionCode: TI

h. country: Myanmar; locality: Shan State; verbatimLatitude: $20^{\circ} 35^{\prime} 41^{\prime \prime} \mathrm{N}$; verbatimLongitude: $96^{\circ} 31^{\prime} 46^{\prime \prime}$ E; eventDate: Nov. 26, 2008; recordedBy: Y. Ito; collectionID: Tanaka et al. 080057; institutionCode: TI

i. country: Myanmar; locality: Shan State; verbatimLatitude: $20^{\circ} 27^{\prime} 28^{\prime \prime} \mathrm{N}$; verbatimLongitude: $96^{\circ} 50^{\prime} 37^{\prime \prime}$ E; eventDate: Dec. 4, 2008; recordedBy: Y. Ito; collectionID: Tanaka et al. 080663; institutionCode: TI

j. country: Myanmar; locality: Taunngyi; verbatimLatitude: $20^{\circ} 47^{\prime} 14 " \mathrm{~N}$; verbatimLongitude: $97^{\circ}$ 2' 7" E; eventDate: Jan. 5, 1938; recordedBy: Y. Ito; collectionID: F.G. Dickason 9334; institutionCode: $\mathrm{GH}$

k. country: Thailand; locality: Chiang Mai Province; Chiang Dao District; verbatimLatitude: $19^{\circ} 28^{\prime} 46 " \mathrm{~N}$; verbatimLongitude: $98^{\circ}$ 54' 44" E; eventDate: Dec. 11, 1992; recordedBy: Y. Ito; collectionID: V.A. Sunthorn, P. Palee 101; institutionCode: GH

I. country: Thailand; verbatimLatitude: $13^{\circ} 45^{\prime} 10^{\prime \prime} \mathrm{N}$; verbatimLongitude: $100^{\circ} 29^{\prime} 45^{\prime \prime} \mathrm{E}$; recordedBy: Y. Ito; collectionID: T- 50413 [TI]; institutionCode: TI 
m. country: Thailand; locality: Pha Team National Park, Ubon Ratchathani, Thailand; verbatimLatitude: $15^{\circ} 24^{\prime} 07^{\prime \prime} \mathrm{N}$; verbatimLongitude: $105^{\circ} 29^{\prime} 20^{\prime \prime} \mathrm{E}$; recordedBy: Y. Ito; collectionID: Tr. Tanaka; institutionCode: TNS

n. country: Thailand; locality: Hotel river Kwai, Kantchanabury; verbatimLatitude: $14^{\circ} 1^{\prime}{ }^{\prime} 59^{\prime \prime}$ $\mathrm{N}$; verbatimLongitude: $99^{\circ} 31^{\prime} 10^{\prime \prime} \mathrm{E}$; eventDate: Nov. 15, 2012; recordedBy: Y. Ito; collectionID: Y. Ito 1724; institutionCode: BKF

Distribution: Bangladesh, Bhutan, China (nationwide), Indonesia (nationwide), Japan, Malaysia (nationwide), Myanmar, Nepal, Pakistan, Papua New Guinea, Thailand, Sri Lanka.

\section{Genus Hydrocharis L., 1753}

\section{Hydrocharis dubia (Blume) Backer, 1925}

\section{Materials}

a. country: Thailand; locality: Ang Thong Province; Howa Pie; verbatimLatitude: $13^{\circ} 54^{\prime} \mathrm{N}$; verbatimLongitude: $100^{\circ} 37^{\prime}$ E; eventDate: Sep. 17, 1972; recordedBy: Y. Ito;

collectionID: J.F. Maxwell 72-398; institutionCode: AAU

b. country: Thailand; locality: Saraburi Province; Muang District, Sahm Lahn forest; verbatimLatitude: $14^{\circ} 31^{\prime} 51^{\prime \prime} \mathrm{N}$; verbatimLongitude: $100^{\circ} 54^{\prime} 34^{\prime \prime} \mathrm{E}$; eventDate: Oct. 20 , 1974; recordedBy: Y. Ito; collectionID: J.F. Maxwell 74-947; institutionCode: AAU

c. country: Thailand; verbatimLatitude: $13^{\circ} 45^{\prime} 10^{\prime \prime} \mathrm{N}$; verbatimLongitude: $100^{\circ} 29^{\prime} 45^{\prime \prime} \mathrm{E}$; recordedBy: Y. Ito; collectionID: 16322; institutionCode: BKF

d. country: Thailand; locality: Maha Sarakham; Bua Khaaw Morning Market; verbatimLatitude: $16^{\circ} 11^{\prime} \mathrm{N}$; verbatimLongitude: $103^{\circ} 18^{\prime} \mathrm{E}$; eventDate: Mar. 11, 1990; recordedBy: Y. Ito; collectionID: Mooly Widmer 0699; institutionCode: BKF

Distribution: China (nationwide), Indonesia (Java, New Guinea, Sulawesi), Japan, ? Myanmar, Papua New Guinea, Philippines, Thailand, ?Vietnam; Oceania.

\section{Genus Najas L., 1753}

\section{Najas graminea Delile, 1813}

\section{Materials}

a. country: Myanmar; locality: Shan State, Inle Lake; verbatimLatitude: $20^{\circ} 32^{\prime} 2^{\prime \prime} \mathrm{N}$; verbatimLongitude: $96^{\circ} 53^{\prime}$ 53" E; eventDate: Dec. 3, 2008; recordedBy: Y. Ito; collectionID: Tanaka et al. 080656; institutionCode: MBK

b. country: Thailand; locality: Mae Hong Son Province; verbatimLatitude: $19^{\circ} 15^{\prime} \mathrm{N}$; verbatimLongitude: $98^{\circ}$ E; eventDate: Sep. 10, 1974; recordedBy: Y. Ito; collectionID: K. Larsen \& S.S. Larsen KL34376; institutionCode: BKF

C. country: Thailand; locality: Pangnga Province; Kan bow koranee cascade; verbatimLatitude: $8^{\circ} 25^{\prime} \mathrm{N}$; verbatimLongitude: $98^{\circ} 30^{\prime} \mathrm{E}$; eventDate: May. 9, 1973; recordedBy: Y. Ito; collectionID: R. Geesink \& T. Santisuk 5299; institutionCode: AAU

d. country: Thailand; locality: Petchabury; verbatimLatitude: $13^{\circ} 30^{\prime} 6 " \mathrm{~N}$; verbatimLongitude: $99^{\circ} 47^{\prime} 37^{\prime \prime}$ E; eventDate: Nov. 14, 2012; recordedBy: Y. Ito; collectionID: Y. Ito 1714; institutionCode: BKF 
Distribution: Bangladesh, China (Central, Southern), India (Central, Southern), Indonesia (Java, New Guinea, Sumatra, Sulawesi), Japan, Myanmar, Nepal, Papua New Guinea, Philippines, Thailand, ?Vietnam; Africa; Oceania.

\section{Najas indica (Willd.) Cham., 1829}

\section{Materials}

a. country: Myanmar; verbatimLatitude: $16^{\circ} 53^{\prime} 19.18 "$; verbatimLongitude: $95^{\circ} 52^{\prime} 28.59^{\prime \prime}$; recordedBy: Y. Ito; collectionID: MBK020915; institutionCode: TI

b. country: Myanmar; verbatimLatitude: $16^{\circ} 53^{\prime} 19.18^{\prime \prime}$; verbatimLongitude: $95^{\circ} 52^{\prime} 28.59^{\prime \prime}$; recordedBy: Y. Ito; collectionID: MBK036320; institutionCode: TI

c. country: Thailand; locality: Ubon Ratchathani Province; Kang Tana Natl park; verbatimLatitude: $15^{\circ} 16^{\prime} 26^{\prime \prime} \mathrm{N}$; verbatimLongitude: 105² 27' 31" E; eventDate: Aug. 22, 2001; recordedBy: Y. Ito; collectionID: R. Pooma et al. 2346; institutionCode: BKF

d. country: Thailand; locality: Chaiyaphum Province; Tunhamang; verbatimLatitude: $16^{\circ} 20^{\prime}$ $\mathrm{N}$; verbatimLongitude: $101^{\circ} 45^{\prime} \mathrm{E}$; eventDate: Dec. 19, 1971; recordedBy: Y. Ito; collectionID: van Beusekom et al. 4245 ; institutionCode: BKF

e. country: Thailand; locality: Petchabury; verbatimLatitude: $13^{\circ} 30^{\prime} 6^{\prime \prime} \mathrm{N}$; verbatimLongitude: $99^{\circ} 47^{\prime} 37^{\prime \prime}$ E; eventDate: Nov. 14, 2012; recordedBy: Y. Ito; collectionID: Y. Ito 1713; institutionCode: BKF

Distribution: Bangladesh, India (Western, Central, Southern), Indonesia (Java, Sumatra, Sulawesi), Myanmar, Papua New Guinea, Sri Lanka, Thailand, Vietnam.

\section{Najas marina L., 1753}

\section{Materials}

a. country: Thailand; locality: Phatthalung Province; Thale noi Bord reservior; verbatimLatitude: $7^{\circ} 44^{\prime} \mathrm{N}$; verbatimLongitude: $100^{\circ}$ 9' E; eventDate: Oct. 7, 2005; recordedBy: Y. Ito; collectionID: H.-J. Esser et al. 05-91; institutionCode: BKF

b. country: Thailand; locality: Phatthalung Province; Sea Thale Noi; verbatimLatitude: $7^{\circ} 47^{\prime}$ 9" N; verbatimLongitude: $100^{\circ} 10^{\prime} 4$ " E; eventDate: Oct. 7, 2005; recordedBy: Y. Ito; collectionID: Tillich et al. 5094; institutionCode: BKF

c. country: Thailand; locality: Khao San Yot Natl Park.; verbatimLatitude: $12^{\circ} 14^{\prime} 39$ " N; verbatimLongitude: $99^{\circ}$ 55' 57" E; eventDate: Nov. 13, 2012; recordedBy: Y. Ito; collectionID: Y. Ito 1701; institutionCode: BKF

Distribution: Worldwide.

\section{Najas tenuis Magnus, 1870}

\section{Materials}

a. country: Myanmar; locality: Shan State; Naungkhio, Hsipaw Distr.; verbatimLatitude: $22^{\circ}$ $37^{\prime} 12^{\prime \prime} \mathrm{N}$; verbatimLongitude: $97^{\circ} 17^{\prime} \mathrm{E}$; eventDate: Oct. 1, 1939; recordedBy: Y. Ito; collectionID: F. G. Dickason 9565; institutionCode: $\mathrm{GH}$

b. country: Myanmar; locality: Shan State, Inle Lake; verbatimLatitude: $20^{\circ} 32^{\prime} 2^{\prime \prime} \mathrm{N}$; verbatimLongitude: $96^{\circ} 53^{\prime} 53^{\prime \prime}$ E; eventDate: Dec. 3, 2008; recordedBy: Y. Ito; collectionID: Tanaka et al. 080642; institutionCode: TI 
Distribution: India, Myanmar.

Notes: Fig. 3.

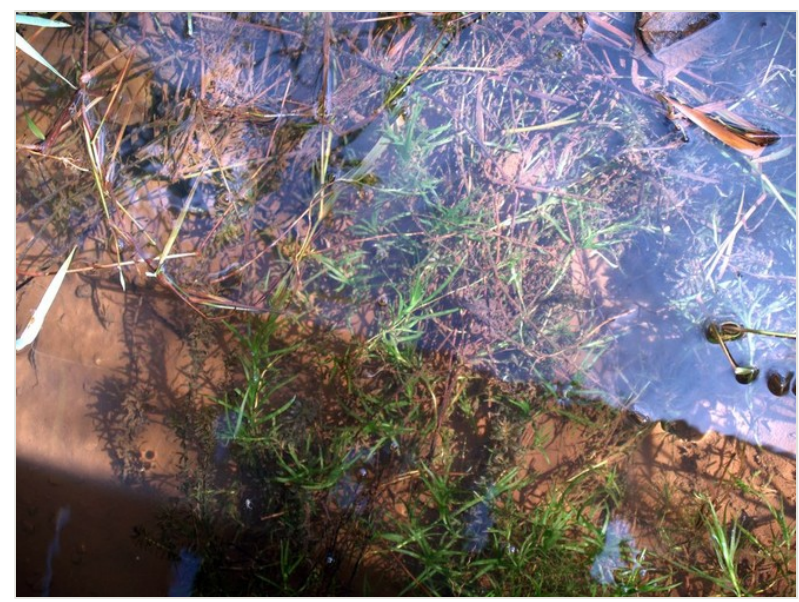

Figure 3.

Najas tenuis in Myanmar (3 DEC 2008). Credit: Y. Ito.

\section{Genus Nechamandra Planch., 1849}

\section{Nechamandra alternifolia (Roxb.) Thwaites, 1864}

\section{Materials}

a. country: Myanmar; locality: Pindaya, Shan State; verbatimLatitude: $20^{\circ} 59^{\prime} 57^{\prime \prime} \mathrm{N}$; verbatimLongitude: $96^{\circ} 39^{\prime} 59^{\prime \prime} \mathrm{E}$; eventDate: Dec. 1, 2008; recordedBy: Y. Ito; collectionID: Tanaka et al. 080635; institutionCode: MBK

b. country: Myanmar; locality: Kalow, Shan State; verbatimLatitude: $20^{\circ} 35^{\prime} 41^{\prime \prime} \mathrm{N}$; verbatimLongitude: $96^{\circ} 31^{\prime} 46^{\prime \prime} \mathrm{E}$; eventDate: Nov. 26, 2008; recordedBy: Y. Ito; collectionID: Tanaka et al. 080053; institutionCode: TI

c. country: Myanmar; locality: Kalow, Shan State; verbatimLatitude: $20^{\circ} 35^{\prime} 41^{\prime \prime} \mathrm{N}$; verbatimLongitude: $96^{\circ} 31^{\prime} 46^{\prime \prime}$ E; eventDate: Nov. 26, 2008; recordedBy: Y. Ito; collectionID: Tanaka et al. 080058; institutionCode: TI

d. country: Myanmar; locality: Kalow, Shan State; verbatimLatitude: $20^{\circ} 35^{\prime} 41^{\prime \prime} \mathrm{N}$; verbatimLongitude: $96^{\circ} 31^{\prime} 46^{\prime \prime} \mathrm{E}$; eventDate: Nov. 26, 2008; recordedBy: Y. Ito; collectionID: Tanaka et al. 080059; institutionCode: TI

e. country: Thailand; locality: Pathum Thani Province; along way to the young delta; verbatimLatitude: $14^{\circ} 1^{\prime} 34^{\prime \prime} \mathrm{N}$; verbatimLongitude: $100^{\circ} 31^{\prime} 29^{\prime \prime} \mathrm{E}$; eventDate: Jul. 30, 1973; recordedBy: Y. Ito; collectionID: G. Murata \& N. Fukuoka T-17324; institutionCode: BKF

Distribution: Bangladesh, China (Southern), India (Eastern, Northern, Southern), Myanmar, Nepal, Sri Lanka, Thailand, Vietnam; Yemen, and Sudan. 
Notes: Fig. 4.

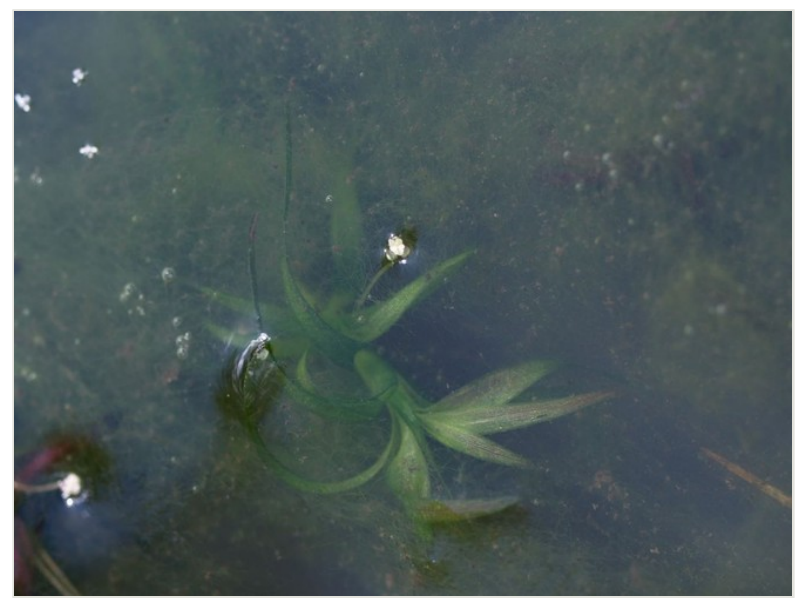

Figure 4.

Nechamandra alternifolia (Hydrocharitaceae) (25 Nov. 2008). Credit: Y. Ito.

Myanmar (Ito et al. 2009); Thailand (Ito 2013b).

\section{Genus Ottelia Pers., 1805}

\section{Ottelia alismoides (L.) Pers., 1805}

\section{Materials}

a. country: Laos; locality: Vientine; verbatimLatitude: $17^{\circ} 58^{\prime} \mathrm{N}$; verbatimLongitude: $102^{\circ} 36^{\prime}$ E; eventDate: Dec. 18, 1957; recordedBy: Y. Ito; collectionID: T. Tsuyama L. 57001; institutionCode: $\mathrm{TI}$

b. country: Myanmar; locality: Mandaley; verbatimLatitude: $21^{\circ} 58^{\prime} 30^{\prime \prime} \mathrm{N}$; verbatimLongitude: $96^{\circ} 5^{\prime} \mathrm{E}$; eventDate: Jan. 14, 1964; recordedBy: Y. Ito; collectionID: H. Kanai; institutionCode: TI

C. country: Myanmar; locality: Kachin State; verbatimLatitude: $26^{\circ} 6^{\prime} 34^{\prime \prime} \mathrm{N}$; verbatimLongitude: $96^{\circ}$ 42' 58" E; eventDate: Sep. 19, 2005; recordedBy: Y. Ito; collectionID: TI040482; institutionCode: TI

d. country: Myanmar; locality: Kachin State; at the evening market of Tanaing, Hukaung Valley, Tanaing Township; verbatimLatitude: $26^{\circ} 31^{\prime} 20^{\prime \prime} \mathrm{N}$; verbatimLongitude: $96^{\circ} 35^{\prime} 9^{\prime \prime}$ E; eventDate: Sep. 20, 2005; recordedBy: Y. Ito; collectionID: TI040488; institutionCode: $\mathrm{TI}$

e. country: Myanmar; locality: Mandalay Division; between Smithine town and Pinchar village, ca. 25 miles S of Mandalay; verbatimLatitude: $21^{\circ} 29^{\prime} 47^{\prime \prime} \mathrm{N}$; verbatimLongitude: $96^{\circ}$ 4' 21" E; eventDate: Jun. 19, 2000; recordedBy: Y. Ito; collectionID: TI2026; institutionCode: $\mathrm{TI}$ 
f. country: Thailand; locality: Chokchai, Nakhon Ratchasima, Thailand; verbatimLatitude: $14^{\circ} 44^{\prime} 30^{\prime \prime} \mathrm{N}$; verbatimLongitude: $102^{\circ} 9^{\prime} 41^{\prime \prime} \mathrm{E}$; recordedBy: Y. Ito; collectionID: Nr. Tanaka; institutionCode: TNS

g. country: Thailand; locality: Petchabury; verbatimLatitude: $13^{\circ} 24^{\prime} 30 " \mathrm{~N}$; verbatimLongitude: $99^{\circ} 48^{\prime} 44^{\prime \prime} \mathrm{E}$; eventDate: Nov. 14, 2012; recordedBy: Y. Ito; collectionID: Y. Ito 1707; institutionCode: BKF

h. country: Thailand; locality: Khuen Shinaga Natl Park.; verbatimLatitude: $14^{\circ} 38^{\prime} 8^{\prime \prime} \mathrm{N}$; verbatimLongitude: $98^{\circ} 59^{\prime} 52^{\prime \prime}$ E; eventDate: Nov. 15, 2012; recordedBy: Y. Ito; collectionID: Y. Ito 1726; institutionCode: BKF

i. country: Thailand; locality: Kantchanabury; verbatimLatitude: $14^{\circ} 39^{\prime} 21^{\prime \prime} \mathrm{N}$; verbatimLongitude: $98^{\circ} 42^{\prime} 27^{\prime \prime}$ E; eventDate: Nov. 15, 2012; recordedBy: Y. Ito; collectionID: Y. Ito 1731; institutionCode: BKF

j. country: Thailand; locality: Nongkhai Province; Bungkhla District, Ban Dong Mak Yang; verbatimLatitude: $18^{\circ} 19^{\prime} 59^{\prime \prime} \mathrm{N}$; verbatimLongitude: $103^{\circ} 43^{\prime} 39^{\prime \prime} \mathrm{E}$; eventDate: Aug. 27 , 2001; recordedBy: Y. Ito; collectionID: R. Pooma, W.J.J.O. de Wilde, B.E.E. Duyfjes, V. Chamchumroon, K. Phattarahirankanok 2864; institutionCode: GH

k. country: Thailand; locality: Kanchanaburi Province; Thong Pha Phum District, Rintin Forest; verbatimLatitude: $14^{\circ} 48^{\prime} 34 " \mathrm{~N}$; verbatimLongitude: $98^{\circ} 44^{\prime} 41^{\prime \prime} \mathrm{E}$; eventDate: Nov. 6, 1979; recordedBy: Y. Ito; collectionID: T. Simizu, H. Toyokuni, H. Koyama, T. Yahara, C. Niyomdham T-21923; institutionCode: GH

I. country: Thailand; locality: Uthaithani Province; Larn Suk District, Huay Kha Kaeng Wildlife Sanctuary; verbatimLatitude: $15^{\circ} 24^{\prime} 30^{\prime \prime} \mathrm{N}$; verbatimLongitude: $100^{\circ} 4^{\prime} 35^{\prime \prime} \mathrm{E}$; eventDate: Nov. 11, 1979; recordedBy: Y. Ito; collectionID: T. Simizu, H. Toyokuni, H. Koyama, T. Yahara, C. Niyomdham T-22221; institutionCode: GH

m. country: Thailand; locality: Chainart Province; verbatimLatitude: $15^{\circ} 14^{\prime} \mathrm{N}$; verbatimLongitude: $100^{\circ} 15^{\prime} \mathrm{E}$; eventDate: Nov. 28, 1959; recordedBy: Y. Ito; collectionID: L.B. \& E.C. Abbe, T. Smitinand, B. Rollet 9233; institutionCode: GH

n. country: Thailand; locality: Songkla Province; Haad Yai District, ban Koke Saht, W of Toong Loong; verbatimLatitude: $7^{\circ} 0^{\prime} 13^{\prime \prime} \mathrm{N}$; verbatimLongitude: $100^{\circ} 27^{\prime} 25^{\prime \prime} \mathrm{E}$; eventDate: Oct. 2, 1985; recordedBy: Y. Ito; collectionID: J.F. Maxwell 85-929; institutionCode: $\mathrm{GH}$

o. country: Thailand; locality: Hue san - Chieing Rae; verbatimLatitude: $19^{\circ} 54^{\prime} 34^{\prime \prime} \mathrm{N}$; verbatimLongitude: $99^{\circ} 49^{\prime} 39^{\prime \prime}$ E; eventDate: Nov. 21, 1921; recordedBy: Y. Ito; collectionID: B. Hayata; institutionCode: TI

Distribution: Cambodia, India, Indonesia, Japan, Korea, Laos, Malaysia, Myanmar, Nepal, New Guinea, Philippines, Thailand, Sri Lanka, Vietnam; Africa, Australia.

\section{Ottelia cordata (Wall.) Dandy, 1934}

\section{Material}

a. country: Myanmar; locality: Mandalay Division; ca. 3 miles from Kyauk Sei, Pyin U Lwin (May Myo); verbatimLatitude: $21^{\circ} 36^{\prime} \mathrm{N}$; verbatimLongitude: $96^{\circ} 4^{\prime} 6^{\prime \prime} \mathrm{E}$; eventDate: Mar. 16, 2003; recordedBy: Y. Ito; collectionID: N. Kuroiwa et al. 020926; institutionCode: TI 
Distribution: ?Cambodia, China (Southern [Hainan]), Myanmar, ?Thailand.

\section{Genus Vallisneria L., 1753}

\section{Vallisneria spiralis L., 1753}

Materials

a. country: Myanmar; locality: Shan State; Inle Lake; verbatimLatitude: $16^{\circ} 53^{\prime} 19^{\prime \prime} \mathrm{N}$; verbatimLongitude: $95^{\circ}$ 52' 29" E; eventDate: Dec. 3, 2008; recordedBy: Y. Ito; collectionID: Tanaka et al. 080653; institutionCode: MBK

b. country: Myanmar; locality: Kachin State; between Khalone and Shinbweyan; verbatimLatitude: $26^{\circ} 40^{\prime} 50^{\prime \prime} \mathrm{N}$; verbatimLongitude: $96^{\circ} 15^{\prime} 20^{\prime \prime} \mathrm{E}$; eventDate: Dec. 5 , 2005; recordedBy: Y. Ito; collectionID: Murata et al.041208; institutionCode: TI

c. country: Myanmar; locality: Kachin State; between Shinbweyan and Tanain; verbatimLatitude: $26^{\circ} 56^{\prime} \mathrm{N}$; verbatimLongitude: $96^{\circ} 52^{\prime} \mathrm{E}$; eventDate: Feb. 16, 2007 ; recordedBy: Y. Ito; collectionID: Murata et al. 041622; institutionCode: TI

Distribution: Bangladesh, China, India (nationwide), Japan, Myanmar, Nepal, Pakistan, Sri Lanka.

\section{Family Aponogetonaceae}

Genus Aponogeton L. f., 1781

\section{Aponogeton lakhonensis A. Camus, 1910}

\section{Materials}

a. country: Myanmar; locality: Kachin State; between Takhet Village and Khalone Village, ca $10 \mathrm{mi}$ E of Shinbwiyang; verbatimLatitude: $26^{\circ} 38^{\prime} 59^{\prime \prime} \mathrm{N}$; verbatimLongitude: $96^{\circ} 20^{\prime} 55^{\prime \prime}$

E; eventDate: Dec. 10, 2005; recordedBy: Y. Ito; collectionID: Tanaka et al. 040939; institutionCode: $\mathrm{TI}$

b. country: Myanmar; locality: Kachin State; along the Ledo Rd between Shinbwiyang and Tanaing; verbatimLatitude: $26^{\circ} 34^{\prime} \mathrm{N}$; verbatimLongitude: $96^{\circ} 30^{\prime} \mathrm{E}$; eventDate: Feb. 16 , 2007; recordedBy: Y. Ito; collectionID: Tanaka et al. 041619; institutionCode: TI

c. country: Thailand; locality: Lampang Province; verbatimLatitude: $18^{\circ} 19^{\prime} 24^{\prime \prime} \mathrm{N}$; verbatimLongitude: 99²9' 29" E; eventDate: Sep. 1, 1926; recordedBy: Y. Ito; collectionID: V.N. Pak Kuap 1797; institutionCode: BKF

Distribution: Cambodia, China (Southern), India (Eastern [Assam, Darjeeling]), Indonesia (Sulawesi), Myanmar, Thailand, Vietnam.

Notes: Fig. 5. 


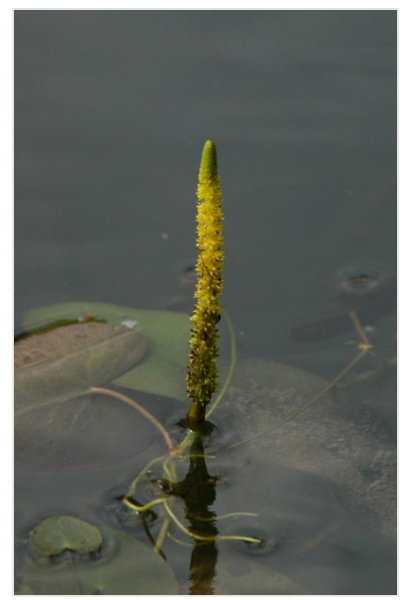

Figure 5.

Aponogeton lakhonensis in Myanmar (10 Dec 2005). Credit: J. Murata.

\section{Family Potamogetonaceae}

Genus Potamogeton L., 1753

\section{Potamogeton crispus L., 1753}

\section{Materials}

a. country: Thailand; locality: Sagaing Division; Kalewa-Kalemyo; verbatimLatitude: $23^{\circ} 12^{\prime}$ 17" N; verbatimLongitude: 94 11' 33" E; eventDate: Mar. 25, 1938; recordedBy: Y. Ito; collectionID: F. G. Dickason 7196; institutionCode: GH

b. country: Myanmar; locality: Sagaing Division; Kalewa-Kalemyo, shallow water, Alt. $600 \mathrm{ft}$;; verbatimLatitude: $23^{\circ} 12^{\prime} 17^{\prime \prime} \mathrm{N}$; verbatimLongitude: 94 11' 33" E; eventDate: Mar. 26 , 1938; recordedBy: Y. Ito; collectionID: F. G. Dickason 7204; institutionCode: GH

Distribution: Bangladesh, Bhutan, China (nationwide), India (nationwide), Japan, Korea, Laos, Malesia (Sumatra), Myanmar, Nepal, Pakistan, Vietnam; Worldwide.

Potamogeton distinctus A. Benn., 1904

\section{Material}

a. country: Thailand; locality: Khuen Shinaga Natl Park; Nearn Saran View point.; verbatimLatitude: $14^{\circ} 44^{\prime} 25^{\prime \prime} \mathrm{N}$; verbatimLongitude: $98^{\circ} 49^{\prime}$ 6" E; eventDate: Nov. 15 , 2012; recordedBy: Y. Ito; collectionID: Y. Ito 1729; institutionCode: BKF 
Potamogeton maackianus A Benn., 1904

\section{Material}

a. country: Myanmar; locality: Shan State; Kalow Village, Yae Aye Kan Dam,; verbatimLatitude: $20^{\circ} 35^{\prime} 41^{\prime \prime} \mathrm{N}$; verbatimLongitude: $96^{\circ} 31^{\prime} 46^{\prime \prime} \mathrm{E}$; eventDate: Nov. 26 , 2008; recordedBy: Y. Ito; collectionID: Nb. Tanaka et al. 080052; institutionCode: TI

Distribution: China (North-eastern, Central, Southern [Yunnan]), Japan, Indonesia (Sumatra), Myanmar, Philippines.

\section{Potamogeton lucens L., 1753}

\section{Materials}

a. country: Myanmar; locality: Shan State; naung Shwe, Inlay Lake; verbatimLatitude: $20^{\circ}$ 36 ' N; verbatimLongitude: $96^{\circ} 54^{\prime} 44^{\prime \prime}$ E; eventDate: May. 2, 2005; recordedBy: Y. Ito; collectionID: K. Khaing 05153; institutionCode: TI

b. country: Myanmar; locality: Shan State, Pindaya, Inlay Lake; verbatimLatitude: $20^{\circ} 59^{\prime}$ $57^{\prime \prime} \mathrm{N}$; verbatimLongitude: $96^{\circ} 39^{\prime}$ 59" E; eventDate: Dec. 1, 2008; recordedBy: Y. Ito; collectionID: Nb. Tanaka et al. 080630; institutionCode: TI

c. country: Myanmar; locality: Inle Lake, Shan State; verbatimLatitude: $16^{\circ} 53^{\prime} 19^{\prime \prime} \mathrm{N}$; verbatimLongitude: $95^{\circ} 52^{\prime} 29 "$ E; eventDate: Dec. 3, 2008; recordedBy: Y. Ito; collectionID: Nb. Tanaka et al. 080640; institutionCode: TI

Distribution: China (North-eastern, Central, Southern [Yunnan]), Japan, Myanmar, Nepal, Philippines; Europe.

\section{Potamogeton nodosus Poir., 1816}

\section{Materials}

a. country: Myanmar; locality: Sagaing Division; Shweuyaung; verbatimLatitude: $24^{\circ} 31^{\prime} 2^{\prime \prime}$ $\mathrm{N}$; verbatimLongitude: $95^{\circ} 23^{\prime} 36 " \mathrm{E}$; eventDate: May. 1, 1938; recordedBy: Y. Ito; collectionID: F. G. Dickason 9374; institutionCode: GH

b. country: Myanmar; locality: Mandalay Division; verbatimLatitude: $20^{\circ} 48^{\prime} \mathrm{N}$; verbatimLongitude: $95^{\circ} 15^{\prime} \mathrm{E}$; eventDate: Nov. 29, 2000; recordedBy: Y. Ito; collectionID: J. Murata et al. 020914; institutionCode: TI

c. country: Myanmar; locality: Chiang Mai Province; Chiang Dao District, Ban Bing kong, Bing Kong subdistrict, in the Bing (Ping) river; verbatimLatitude: $19^{\circ} 36^{\prime} 9^{\prime \prime} \mathrm{N}$; verbatimLongitude: $98^{\circ} 55^{\prime} 49^{\prime \prime} \mathrm{E}$; eventDate: Dec. 6, 1992; recordedBy: Y. Ito; collectionID: J.F. Maxwell 92-803; institutionCode: AAU

d. country: Thailand; locality: Chiang Mai; old Mae Ping (Bing) dam, along the Ping (Bing) river; verbatimLatitude: $19^{\circ} 36^{\prime}$ 9" N; verbatimLongitude: $98^{\circ} 55^{\prime} 49 " \mathrm{E}$; eventDate: Jul. 6 , 1995; recordedBy: Y. Ito; collectionID: P. Thavoeburum 1; institutionCode: GH

e. country: Thailand; locality: Chiang Mai; Ban Bing kong, Bing Kong subdistrict, in the Bing (Ping) river; verbatimLatitude: $19^{\circ} 36^{\prime} 9^{\prime \prime} \mathrm{N}$; verbatimLongitude: $98^{\circ} 55^{\prime} 49^{\prime \prime} \mathrm{E}$; eventDate: Dec. 6, 1992; recordedBy: Y. Ito; collectionID: J.F. Maxwell 92-605; institutionCode: GH

f. country: Thailand; locality: Chiang Mai Province; Mae Dtang District, in the Bing (Ping) river; verbatimLatitude: $19^{\circ} 36^{\prime}$ 9" N; verbatimLongitude: $98^{\circ} 55^{\prime} 49^{\prime \prime} \mathrm{E}$; eventDate: Dec. 11, 1992; recordedBy: Y. Ito; collectionID: J.F. Maxwell 92-832; institutionCode: AAU 
g. country: Thailand; locality: Chiang Rai Province; Santonthong, Muang, Lampun; verbatimLatitude: $19^{\circ} 37^{\prime} 41^{\prime \prime} \mathrm{N}$; verbatimLongitude: $100^{\circ}$ 4' 16" E; eventDate: Apr. 20 , 1971; recordedBy: Y. Ito; collectionID: P. Karnchanomai s.n.; institutionCode: AAU

Distribution: Bangladesh, China (North-Eastern, Central [in part], Southern [Yunnan]), India (nationwide), Indonesia (New Guinea, Sumatra, Sulawesi), Myanmar, Nepal, Pakistan, Philippines, Sri Lanka, Thailand, Vietnam; Africa, Europe, America.

\section{Potamogeton octandrus Poir., 1816}

\section{Materials}

a. country: Myanmar; locality: Chiang Mai Province; Haung Dong, route to Khan Waang; verbatimLatitude: $18^{\circ} 42^{\prime} 58^{\prime \prime} \mathrm{N}$; verbatimLongitude: $98^{\circ} 55^{\prime} 35^{\prime \prime} \mathrm{E}$; eventDate: Apr. 5 , 1978; recordedBy: Y. Ito; collectionID: T. Smitinand s.n.; institutionCode: BKF

b. country: Thailand; locality: Kachin State; between Khalone Village and Shinbweyan; verbatimLatitude: $26^{\circ} 40^{\prime} 29^{\prime \prime} \mathrm{N}$; verbatimLongitude: $96^{\circ} 16^{\prime} 33^{\prime \prime} \mathrm{E}$; eventDate: Dec. 5 , 2005; recordedBy: Y. Ito; collectionID: J. Murata et al. 040649; institutionCode: TI

Distribution: Bangladesh, Bhutan, China (nationwide), Japan, India (Northern, Eastern, Southern [in part]), Indonesia (Java), Myanmar, Nepal, Papua New Guinea, Thailand, Vietnam; Africa; Oceania.

Notes: Myanmar (Ito et al. 2009); Thailand (Ito 2013b).

\section{Potamogeton wrightii Morong, 1886}

\section{Materials}

a. country: Myanmar; locality: Mandalay Division; Kyet Mank Taung Dam, Kyaukpadaung Township, Popa; verbatimLatitude: $20^{\circ} 48^{\prime} \mathrm{N}$; verbatimLongitude: $95^{\circ} 15^{\prime} \mathrm{E}$; eventDate: Mar. 4, 2003; recordedBy: Y. Ito; collectionID: Kuroiwa et al. 028702; institutionCode: TI

b. country: Thailand; locality: Songkla Province; Satingpra District, Bantom island, Lake Songkla, ca. $100 \mathrm{~m}$ offshore.; verbatimLatitude: $7^{\circ} 28^{\prime} \mathrm{N}$; verbatimLongitude: $100^{\circ} 24^{\prime} \mathrm{E}$; eventDate: Sep. 22, 1984; recordedBy: Y. Ito; collectionID: J.F. Maxwell 84-233; institutionCode: AAU

Distribution: Bangradesh, China (nationwide), India (Northern), Indonesia (Celebes, Moluccas, Lonbok, Sumatra), Japan, Malaysia (Borneo, Peninsular), Myanmar, Pakistan, Philippines, Thailand, Vietnam. 


\section{Genus Stuckenia Börner, 1912}

\section{Stuckenia pectinata (L.) Börner, 1912}

Material

a. country: Myanmar; locality: Shan State; Inlay Lake; verbatimLatitude: $20^{\circ} 36^{\prime} \mathrm{N}$; verbatimLongitude: $96^{\circ}$ 54' 44" E; eventDate: Nov. 13, 2005; recordedBy: Y. Ito; collectionID: K. Khaing 05210; institutionCode: TI

Distribution: Bangladesh, Bhutan, China (nationwide), India (nationwide), Indonesia (Sumatra, Sulawesi), Japan, Myanmar, Nepal, Pakistan, Sri Lanka; Worldwide.

\section{Family Ruppiaceae}

\section{Genus Ruppia L., 1753}

\section{Ruppia maritima L., 1753}

\section{Material}

a. country: Thailand; locality: Khao San Yot Natl Park.; verbatimLatitude: $12^{\circ} 9^{\prime} 15^{\prime \prime} \mathrm{N}$; verbatimLongitude: $99^{\circ} 58^{\prime} 44^{\prime \prime}$ E; eventDate: Nov. 13, 2012; recordedBy: Y. Ito; collectionID: Y. Ito 1706; institutionCode: BKF

Distribution: Bangladesh, China (North-Eastern, Central, Southern), India (Southern), ?Myanmar, Nepal, Sri Lanka, Thailand, Vietnam; Worldwide.

\section{Order Asparagales}

\section{Family Amaryllidaceae}

Genus Crinum L., 1753

\section{Crinum thaianum J. Schul., 1971}

\section{Materials}

\section{Other materials:}

a. country: Thailand; locality: Phang Nga Province; Klong Tam Nung; verbatimLatitude: $8^{\circ}$ 27' 22" N; verbatimLongitude: $98^{\circ} 31^{\prime} 8^{\prime \prime} \mathrm{E}$; recordedBy: Y. Ito; collectionID: C. Niyomdham 1256; institutionCode: AAU

b. country: Thailand; locality: Phang Nga Province; Ban Nang Yon, 30 miles $\mathrm{N}$ of Takua Pa, on the way to Ranong; verbatimLatitude: $9^{\circ} 12^{\prime} 20^{\prime \prime} \mathrm{N}$; verbatimLongitude: $98^{\circ} 25^{\prime} 28^{\prime \prime} \mathrm{E}$; eventDate: Apr. 18, 1970; recordedBy: Y. Ito; collectionID: J. Schulze 357; institutionCode: MO 
Holotype:

a. country: Thailand; locality: Phang Nga Province; Ban Nang Yon, 30 miles N of Takua Pa, on the way to Ranong; eventDate: Apr. 18, 1970; recordedBy: Y. Ito; collectionID: J. Schulze 1011; institutionCode: US

Distribution: Thailand.

Notes: Lectotype was designated by Lekhak and Yadav (2012).

Fig. 6.

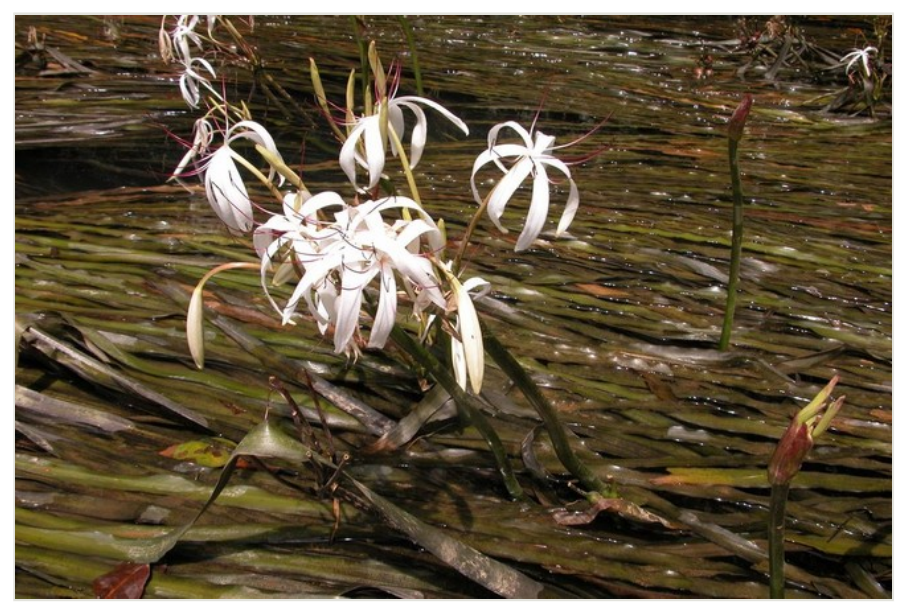

Figure 6.

Crinum thaianum in Thailand (3 Dec. 2003). Credit: R. Pooma.

\section{Order Commelinales}

\section{Family Pontederiaceae}

\section{Genus Eichhornia Kunth, 1843}

\section{Eichhornia crassipes (Mart.) Solms, 1883}

Materials

a. country: Myanmar; locality: Rangoon; verbatimLatitude: $16^{\circ} 49^{\prime} 37^{\prime \prime} \mathrm{N}$; verbatimLongitude: 96 8' 58" E; eventDate: Jan. 2, 1938; recordedBy: Y. Ito; collectionID: F.G. Dickason 6966; institutionCode: $\mathrm{GH}$

b. country: Myanmar; locality: Wakema; verbatimLatitude: $16^{\circ} 36^{\prime} 18^{\prime \prime} \mathrm{N}$; verbatimLongitude: 95 10' 49" E; eventDate: Jan. 7, 1938; recordedBy: Y. Ito; collectionID: F.G. Dickason 7820; institutionCode: $\mathrm{GH}$

c. country: Myanmar; locality: Shan State; Inle lake; verbatimLatitude: $20^{\circ} 32^{\prime} 2^{\prime \prime} \mathrm{N}$; verbatimLongitude: $96^{\circ}$ 53' 53" E; eventDate: Dec. 3, 2008; recordedBy: Y. Ito; collectionID: Tanaka et al. 080645; institutionCode: MBK 
d. country: Thailand; locality: Songkla Province, Talae Noi Waterfall Reserve, $\mathrm{N}$ end of Lake Songkla; verbatimLatitude: $7^{\circ} 15^{\prime} \mathrm{N}$; verbatimLongitude: $100^{\circ} 26^{\prime} 16^{\prime \prime} \mathrm{E}$; eventDate: Dec. 28, 1978; recordedBy: Y. Ito; collectionID: G. Congdon 162; institutionCode: AAU

e. country: Thailand; locality: Songkla Province; Talae Noi waterfowl reserve, $\mathrm{N}$ end of lake Songkla, near Phattalung; verbatimLatitude: $7^{\circ} 15^{\prime} \mathrm{N}$; verbatimLongitude: $100^{\circ} 26^{\prime} 16^{\prime \prime} \mathrm{E}$; eventDate: Dec. 28, 1978; recordedBy: Y. Ito; collectionID: G. Congdon \& C. Hamilton 162; institutionCode: GH

Distribution: Native to South America.

\section{Genus Monochoria C. Presl, 1827}

\section{Monochoria elata Ridl., 1918}

\section{Materials}

a. country: Thailand; locality: Nakhon Si Thamarat Province; Phru Kuan Kleng; verbatimLatitude: $8^{\circ} 26^{\prime} 7 " \mathrm{~N}$; verbatimLongitude: $99^{\circ} 57^{\prime} 45^{\prime \prime} \mathrm{E}$; eventDate: Jan. 26 , 1999; recordedBy: Y. Ito; collectionID: C. Niyomdham 5662; institutionCode: BKF

b. country: Thailand; locality: Phatthalung Province; Khuan Khanun; verbatimLatitude: $7^{\circ} 44^{\prime}$ 6" N; verbatimLongitude: $100^{\circ}$ 0' 36" E; eventDate: Dec. 24, 2006; recordedBy: Y. Ito; collectionID: R. Pooma et al. 6609; institutionCode: BKF

c. country: Thailand; locality: Patalung Province; Thale Luang; verbatimLatitude: $7^{\circ} 36^{\prime} \mathrm{N}$; verbatimLongitude: $100^{\circ} 10^{\prime} \mathrm{E}$; eventDate: Mar. 21, 1960; recordedBy: Y. Ito; collectionID: L.B. \& E.C. Abbe et al. 9698; institutionCode: BKF

d. country: Thailand; locality: Saraburi Province.; verbatimLatitude: $9^{\circ} 16^{\prime} \mathrm{N}$; verbatimLongitude: $99^{\circ} 32$ ' E; eventDate: Oct. 11, 1964; recordedBy: Y. Ito; collectionID: T. Smitinand 29879; institutionCode: BKF

e. country: Thailand; locality: Chachoengsao Province; near Phanom Sarakhan, 50km E of Chachoengsao; verbatimLatitude: $13^{\circ} 40^{\prime} \mathrm{N}$; verbatimLongitude: $101^{\circ} 30^{\prime} \mathrm{E}$; eventDate: Oct. 1, 1984; recordedBy: Y. Ito; collectionID: G. Murata et al. T-37035; institutionCode: BKF

Distribution: Malaysia (Peninsular), Thailand.

\section{Monochoria hastata (L.) Solms, 1883}

\section{Materials}

a. country: Myanmar; locality: Rangoon; verbatimLatitude: $16^{\circ} 49^{\prime} 37^{\prime \prime} \mathrm{N}$; verbatimLongitude: $96^{\circ}$ 8' 58" E; eventDate: Nov. 1, 1937; recordedBy: Y. Ito; collectionID: F.G. Dickason 6665; institutionCode: $\mathrm{GH}$

b. country: Myanmar; locality: Rangoon; verbatimLatitude: $16^{\circ} 49^{\prime} 37^{\prime \prime}$; verbatimLongitude: $96^{\circ} 8^{\prime}$ 58" E; eventDate: Jan. 2, 1938; recordedBy: Y. Ito; collectionID: F.G. Dickason 7135; institutionCode: $\mathrm{GH}$

c. country: Myanmar; locality: Shan State; Inle Lake; verbatimLatitude: $20^{\circ} 35^{\prime} 21^{\prime \prime} \mathrm{N}$; verbatimLongitude: $96^{\circ} 54^{\prime} 35^{\prime \prime}$ E; eventDate: Jan. 5, 1938; recordedBy: Y. Ito; collectionID: F.G. Dickason 7873; institutionCode: $\mathrm{GH}$

d. country: Thailand; locality: Sakon Nakhon Province; Nong Han Pond; verbatimLatitude: $17^{\circ} 15^{\prime} 16^{\prime \prime} \mathrm{N}$; verbatimLongitude: $104^{\circ}$ 9' 43" E; eventDate: Aug. 25, 2001; recordedBy: 
Y. Ito; collectionID: R. Pooma, W.J.J.O. de Wilde, B.E.E. Duyfjes, V. Chamchumroon, K. Phattarahirankanok 2579; institutionCode: $\mathrm{GH}$

e. country: Thailand; locality: Chiang Mai Province; Ching Hao District, Doi Ching Dao; verbatimLatitude: $19^{\circ} 22^{\prime} 7^{\prime \prime} \mathrm{N}$; verbatimLongitude: $98^{\circ} 56^{\prime} 47^{\prime \prime} \mathrm{E}$; eventDate: Sep. 30, 1989; recordedBy: Y. Ito; collectionID: J.F. Maxwell 89-1153; institutionCode: GH

f. country: Thailand; locality: Lampang Province; Chae Hom District, N part of the forest at Jaehomwittaya school; verbatimLatitude: $18^{\circ} 41^{\prime} \mathrm{N}$; verbatimLongitude: $99^{\circ} 38^{\prime} \mathrm{E}$; eventDate: Aug. 6, 2000; recordedBy: Y. Ito; collectionID: M. Panatkool 316; institutionCode: $\mathrm{GH}$

g. country: Thailand; locality: Rayong Province; verbatimLatitude: $12^{\circ} 41^{\prime} 38^{\prime \prime} \mathrm{N}$; verbatimLongitude: $101^{\circ} 13^{\prime} 49^{\prime \prime}$ E; eventDate: Aug. 21, 1977; recordedBy: Y. Ito; collectionID: C. Phengklai et al. 3782; institutionCode: GH

h. country: Thailand; locality: Chiang Mai Province; Phek Khaeng Kai; verbatimLatitude: $18^{\circ}$ 47' 30" N; verbatimLongitude: $98^{\circ}$ 57' 38" E; eventDate: Feb. 20, 1991; recordedBy: Y. Ito; collectionID: R. Pooma 91033; institutionCode: GH

i. country: Thailand; locality: Narathiwat Province; Paa Wai, Su Ngai Paadee; verbatimLatitude: $6^{\circ} 7^{\prime} 37^{\prime \prime} \mathrm{N}$; verbatimLongitude: $101^{\circ} 54$ ' 47" E; eventDate: Aug. 24, 1988; recordedBy: Y. Ito; collectionID: C. Niyomdham \& W. Ueachirakan 1910; institutionCode: AAU

j. country: Thailand; locality: Kalasin Province; Muang Kalasin District.; verbatimLatitude: $16^{\circ} 20^{\prime} \mathrm{N}$; verbatimLongitude: $103^{\circ} 32^{\prime} \mathrm{E}$; eventDate: Mar. 13, 1990; recordedBy: Y. Ito; collectionID: C. Phengklai 0077; institutionCode: BKF

k. country: Thailand; locality: Phatthalung Province;nok Nam Thale Noi Bird Sanctuary; verbatimLatitude: $7^{\circ} 47^{\prime} \mathrm{N}$; verbatimLongitude: $100^{\circ} 7^{\prime} \mathrm{E}$; eventDate: Sep. 1, 1996; recordedBy: Y. Ito; collectionID: P. Wilkin 845; institutionCode: BKF

I. country: Thailand; locality: Sakon Nakhon Province; Nong han pond.; verbatimLatitude: $17^{\circ} 15^{\prime} 16^{\prime \prime} \mathrm{N}$; verbatimLongitude: $104^{\circ}$ 9' 43" E; eventDate: Aug. 25, 2001; recordedBy: Y. Ito; collectionID: R. Pooma et al. 2579; institutionCode: BKF

m. country: Thailand; locality: Rayong Province.; verbatimLatitude: $12^{\circ} 41^{\prime} 38^{\prime \prime} \mathrm{N}$; verbatimLongitude: $101^{\circ} 13^{\prime} 49^{\prime \prime}$ E; eventDate: Aug. 21, 1977; recordedBy: Y. Ito; collectionID: C. Phengklai et al. 71314; institutionCode: BKF

n. country: Thailand; locality: Krungtep Phra Nakhon Province; Soi Somprasong.; verbatimLatitude: $13^{\circ} 46^{\prime} 36^{\prime \prime} \mathrm{N}$; verbatimLongitude: $100^{\circ} 30^{\prime} \mathrm{E}$; eventDate: Jan. 31 , 1966; recordedBy: Y. Ito; collectionID: N. Fukuoka 7075; institutionCode: BKF

Distribution: Bhutan, Bangladesh, ?Cambodia, China (South), India (nationwide), Myanmar, Nepal, Papua New Guinea, Sri Lanka, Thailand, ?Vietnam.

Notes: Fig. 7. 


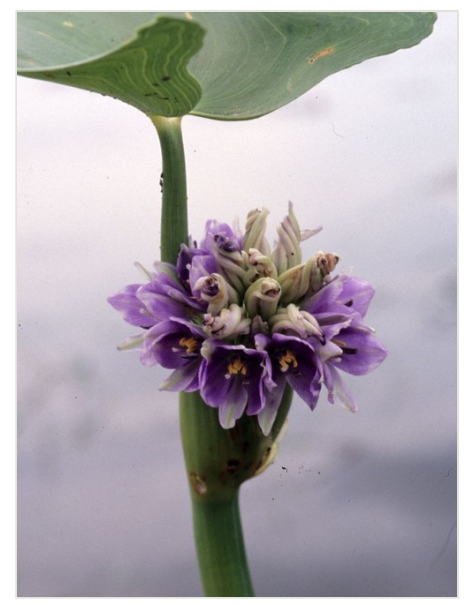

Figure 7.

Monochoria hastata in Thailand (5 Sep. 2008). Credit: Udon.

\section{Monochoria vaginalis (Burm.f.) C. Presl ex Kunth, 1843}

\section{Materials}

a. country: Myanmar; locality: Shan State; Inlay Lake, Pindaya; verbatimLatitude: $20^{\circ} 59^{\prime}$ $57 " \mathrm{~N}$; verbatimLongitude: $96^{\circ} 39^{\prime}$ 59" E; eventDate: Dec. 1, 2008; recordedBy: Y. Ito; collectionID: Tanaka et al. 080637; institutionCode: MBK

b. country: Myanmar; locality: Rangoon; verbatimLatitude: $16^{\circ} 49^{\prime} 37^{\prime \prime} \mathrm{N}$; verbatimLongitude: $96^{\circ} 8^{\prime} 58^{\prime \prime}$ E; eventDate: Jan. 10, 1937; recordedBy: Y. Ito; collectionID: F.G. Dickason 6538; institutionCode: $\mathrm{GH}$

c. country: Myanmar; locality: Kachin State; Tanaing, Hukaung valley; verbatimLatitude: $26^{\circ}$ 6' 34" N; verbatimLongitude: $96^{\circ} 42^{\prime}$ 58" E; eventDate: Sep. 19, 2005; recordedBy: Y. Ito; collectionID: Tanaka et al. 040481; institutionCode: TI

d. country: Myanmar; locality: Shan State; Yae Aye Kan; verbatimLatitude: $20^{\circ} 35^{\prime} 41^{\prime \prime} \mathrm{N}$; verbatimLongitude: $96^{\circ} 31^{\prime} 46^{\prime \prime} \mathrm{E}$; eventDate: Nov. 26, 2008; recordedBy: Y. Ito; collectionID: Tanaka et al. 080056; institutionCode: TI

e. country: Thailand; locality: Narathiwat Province; Paa Waai, Su Ngi Paadee; verbatimLatitude: $6^{\circ} 7^{\prime} 37^{\prime \prime} \mathrm{N}$; verbatimLongitude: $101^{\circ} 54^{\prime} 52$ " E; eventDate: Sep. 4 , 1987; recordedBy: Y. Ito; collectionID: C. Niyomdham \& D. Sriboonma 1543; institutionCode: AAU

f. country: Thailand; locality: Narathiwat Province; Kok Kun Bet, Tak Bai; verbatimLatitude: $6^{\circ} 15^{\prime} 31^{\prime \prime} \mathrm{N}$; verbatimLongitude: $102^{\circ} 0^{\prime}$ 6" E; eventDate: Feb. 17, 1988; recordedBy: Y. Ito; collectionID: C. Niyomdham 1698; institutionCode: AAU

g. country: Thailand; locality: Trat Province; Saphan Hin; verbatimLatitude: $12^{\circ} 0^{\prime} \mathrm{N}$; verbatimLongitude: $102^{\circ}$ 40' E; eventDate: May. 3, 1974; recordedBy: Y. Ito; collectionID: R. Geesink et al. 6510; institutionCode: AAU

h. country: Thailand; locality: Chanthabury Province; verbatimLatitude: $13^{\circ} 3^{\prime} \mathrm{N}$; verbatimLongitude: $101^{\circ} 56^{\prime} \mathrm{E}$; eventDate: Oct. 17, 1971; recordedBy: Y. Ito; collectionID: J.F. Maxwell 71-553; institutionCode: AAU 
i. country: Thailand; locality: Chonbury Province, Sattahip District; taang Brang; verbatimLatitude: $12^{\circ} 40^{\prime} \mathrm{N}$; verbatimLongitude: $100^{\circ}$ 55' E; eventDate: Dec. 4, 1972; recordedBy: Y. Ito; collectionID: J.F. Maxwell 72-600; institutionCode: AAU

j. country: Thailand; locality: Chonburi Province; Siricha District, Kow Kieo; verbatimLatitude: $13^{\circ} 14^{\prime} 15^{\prime \prime} \mathrm{N}$; verbatimLongitude: $101^{\circ} 2^{\prime}$ 32" E; eventDate: Sep. 4 , 1975; recordedBy: Y. Ito; collectionID: J.F. Maxwell 75-979; institutionCode: AAU

k. country: Thailand; locality: Songkla Province; Haad Yai District, Klong Hoy Kong, W of Toong Loong; verbatimLatitude: $7^{\circ} 0^{\prime} 13^{\prime \prime} \mathrm{N}$; verbatimLongitude: $100^{\circ} 27^{\prime} 25$ " e; eventDate: May. 30, 1985; recordedBy: Y. Ito; collectionID: J.F. Maxwell 85-553; institutionCode: AAU

I. country: Thailand; locality: Songkhla Province; verbatimLatitude: $6^{\circ} 37^{\prime} \mathrm{N}$; verbatimLongitude: $100^{\circ}$ 56' E; eventDate: Oct. 8, 1988; recordedBy: Y. Ito; collectionID: K. Larsen \& S.S. Larsen 10290; institutionCode: AAU

m. country: Thailand; locality: Uttaradit Province; Road 1047 from Nam Pat ro Boh Bia; verbatimLatitude: $17^{\circ} 43^{\prime} \mathrm{N}$; verbatimLongitude: $100^{\circ} 36^{\prime} \mathrm{E}$; eventDate: Sep. 21, 1996; recordedBy: Y. Ito; collectionID: P.C. Boyce 1121; institutionCode: AAU

n. country: Thailand; locality: Nong Khai Province; Se Ka District, Tha Sa-sd; verbatimLatitude: $18^{\circ} 19^{\prime} 59^{\prime \prime} \mathrm{N}$; verbatimLongitude: $103^{\circ} 43^{\prime} 39^{\prime \prime} \mathrm{E}$; eventDate: Aug. 28 , 2001; recordedBy: Y. Ito; collectionID: R. Pooma, W.J.J.O. de Wilde, B.E.E. Duyfjes, V. Chamchumroon, K. Phattarahirankanok 2874; institutionCode: GH

o. country: Thailand; locality: Phang Nga Province; Koh Kaw Khao island; verbatimLatitude: $9^{\circ} 0^{\prime} \mathrm{N}$; verbatimLongitude: $98^{\circ} 50^{\prime} \mathrm{E}$; eventDate: Jul. 15, 1972; recordedBy: Y. Ito; collectionID: K. Larsen et al. 30991; institutionCode: AAU

p. country: Thailand; locality: Chaiyaphum Province; Dat Don; verbatimLatitude: $15^{\circ} 57^{\prime} \mathrm{N}$; verbatimLongitude: $102^{\circ} 2^{\prime}$ E; eventDate: Aug. 13, 1972; recordedBy: Y. Ito; collectionID: K. Larsen et al. 31774; institutionCode: AAU

q. country: Thailand; locality: Ranong Province; NW of Phato; verbatimLatitude: $9^{\circ} 50$ ' N; verbatimLongitude: $98^{\circ}$ 40' E; eventDate: May. 2, 1974; recordedBy: Y. Ito; collectionID: K. Larsen \& S.S. Larsen 33543; institutionCode: AAU

r. country: Thailand; locality: Maehongson Province; Khun Yuam; verbatimLatitude: $18^{\circ} 15^{\prime}$ $\mathrm{N}$; verbatimLongitude: $98^{\circ}$ 0' E; eventDate: Sep. 7, 1974; recordedBy: Y. Ito; collectionID: K. Larsen \& S.S. Larsen 34249; institutionCode: AAU

s. country: Thailand; locality: Phitsanulok Province; Tung Salaeng Luang; verbatimLatitude: $16^{\circ} 59^{\prime} \mathrm{N}$; verbatimLongitude: $100^{\circ} 53^{\prime} \mathrm{E}$; eventDate: Jul. 24,1966 ; recordedBy: Y. Ito; collectionID: K. Larsen et al. 833; institutionCode: AAU

t. country: Thailand; locality: Phuket Province; W of Ban Bo Han; verbatimLatitude: $8^{\circ} 7^{\prime} \mathrm{N}$; verbatimLongitude: $98^{\circ} 18^{\prime} \mathrm{E}$; eventDate: Oct. 8, 1970; recordedBy: Y. Ito; collectionID: C. Charoenphol et al. 3422; institutionCode: AAU

u. country: Thailand; locality: Mahasarakarm Province; Koksung District, Amphur Chiengyeun; verbatimLatitude: $16^{\circ} 11^{\prime} 18^{\prime \prime} \mathrm{N}$; verbatimLongitude: $103^{\circ} 17^{\prime} 58^{\prime \prime} \mathrm{E}$; eventDate: Sep. 18, 1984; recordedBy: Y. Ito; collectionID: N. Fukuoka T-36166; institutionCode: $\mathrm{GH}$

v. country: Thailand; locality: Chiang Mai Province; Doi Inthanon, N side of the route RS-13; verbatimLatitude: $18^{\circ} 32^{\prime} 23^{\prime \prime} \mathrm{N}$; verbatimLongitude: $98^{\circ} 31^{\prime} 15^{\prime \prime} \mathrm{E}$; eventDate: Jul. 22 , 1988; recordedBy: Y. Ito; collectionID: S. Tsugaru T-61719; institutionCode: GH

w. country: Thailand; locality: Chachoengsao Province; Chachoengsao Wildlife Reserve; verbatimLatitude: $13^{\circ} 26^{\prime} \mathrm{N}$; verbatimLongitude: $101^{\circ} 55^{\prime}$ E; eventDate: Sep. 11, 1999; recordedBy: Y. Ito; collectionID: D.J. middleton, W. Sangkamethawee 227; institutionCode: $\mathrm{GH}$ 
x. country: Thailand; locality: Krabi Province; Klongtom District, Ban Klong Rat; verbatimLatitude: $7^{\circ} 55^{\prime} 48^{\prime \prime} \mathrm{N}$; verbatimLongitude: $99^{\circ} 13^{\prime} 42^{\prime \prime} \mathrm{E}$; eventDate: Nov. 29 , 1986; recordedBy: Y. Ito; collectionID: J.F. Maxwell 86-991; institutionCode: GH

y. country: Thailand; locality: Petchabury; verbatimLatitude: $13^{\circ} 30^{\prime} 6^{\prime \prime} \mathrm{N}$; verbatimLongitude: $99^{\circ} 47^{\prime} 37^{\prime \prime} \mathrm{E}$; eventDate: 11-14-12; recordedBy: Y. Ito; institutionCode: BKF

Z. country: Vietnum; locality: Kontum Province; verbatimLatitude: $14^{\circ} 42^{\prime} 40^{\prime \prime} \mathrm{N}$; verbatimLongitude: $107^{\circ} 49^{\prime} 48^{\prime \prime} \mathrm{E}$; eventDate: Apr. 14, 1995; recordedBy: Y. Ito; collectionID: L. Averyanov et al.VH1360; institutionCode: AAU

aa. country: Vietnum; locality: Kon Tum Province; Dak Gley District; verbatimLatitude: $15^{\circ} 6^{\prime}$ $57 " \mathrm{~N}$; verbatimLongitude: $107^{\circ} 42^{\prime} 55^{\prime \prime} \mathrm{E}$; eventDate: Nov. 22, 1995; recordedBy: Y. Ito; collectionID: L. Averyanov et al. VH1906; institutionCode: AAU

ab. country: Vietnum; locality: Lam Dong Province; Lac Dung District; verbatimLatitude: $12^{\circ} 8^{\prime}$ $\mathrm{N}$; verbatimLongitude: $108^{\circ} 39^{\prime} \mathrm{E}$; eventDate: Apr. 9, 1997; recordedBy: Y. Ito; collectionID: L. Averyanov et al. VH3814; institutionCode: AAU

ac. country: Vietnum; locality: Lam Dong Province; Lac Duong District; verbatimLatitude: $12^{\circ}$ 8' N; verbatimLongitude: $108^{\circ} 39^{\prime} \mathrm{E}$; eventDate: Apr. 21, 1997; recordedBy: Y. Ito; collectionID: L. Averyanov et al. VH3896; institutionCode: AAU

Distribution: Bangladesh, Bhutan, China (nationwide), India (nationwide), ?Indonesia, Japan, Korea, ?Malaysia, Myanmar, Nepal, Papua New Guinea, Pakistan, Philippines, Sri Lanka, Thailand; Africa; Oceania.

\section{Family Typhaceae}

\section{Genus Typha L., 1753}

\section{Typha angustifolia L., 1753}

\section{Materials}

a. country: Thailand; locality: Chantabury Province; Klang (between bangkok and Paknam); verbatimLatitude: $12^{\circ} 16^{\prime} \mathrm{N}$; verbatimLongitude: $102^{\circ} 20^{\prime} \mathrm{E}$; eventDate: Mar. 11, 1958; recordedBy: Y. Ito; collectionID: Kai Larsen 2053; institutionCode: GH

b. country: Thailand; locality: Chonburi Province; Siracha District, Siracha island; verbatimLatitude: $13^{\circ} 8^{\prime} 56^{\prime \prime} \mathrm{N}$; verbatimLongitude: $100^{\circ} 48^{\prime} 37^{\prime \prime} \mathrm{E}$; eventDate: Nov. 29 , 1992; recordedBy: Y. Ito; collectionID: J.F. Maxwell 92-776; institutionCode: GH

C. country: Thailand; locality: Petchabury; verbatimLatitude: $13^{\circ} 24^{\prime} 30^{\prime \prime} \mathrm{N}$; verbatimLongitude: $99^{\circ} 48^{\prime} 44^{\prime \prime}$ E; eventDate: Nov. 14, 2012; recordedBy: Y. Ito; collectionID: Y. Ito 1708; institutionCode: BKF

d. country: Thailand; verbatimLatitude: $13^{\circ} 45^{\prime} \mathrm{N}$; verbatimLongitude: $100^{\circ} 29^{\prime} \mathrm{E}$; recordedBy: Y. Ito; collectionID: M. Tagawa \& I. Yamada T 112; institutionCode: TI

Distribution: Bangladesh, China (nationwide), India (nationwide), Japan, ?Myanmar, Pakistan; Worldwide. 


\section{Order Poales}

\section{Family Eriocaulaceae}

\section{Genus Eriocaulon L., 1753}

\section{Eriocaulon setaceum L., 1753}

Materials

a. country: Thailand; locality: Sakon Nakhon; Thamphura Fall; verbatimLatitude: $17^{\circ} 9^{\prime} 15^{\prime \prime}$ $\mathrm{N}$; verbatimLongitude: $104^{\circ} 8^{\prime} 10^{\prime \prime} \mathrm{E}$; eventDate: Jul. 22, 2003; recordedBy: Y. Ito; collectionID: Th. Wongprasert et al. 037-15; institutionCode: BKF

b. country: Thailand; locality: Phangnga, Khuraburi, Ko Phrathong; verbatimLatitude: $9^{\circ} 6^{\prime}$ $\mathrm{N}$; verbatimLongitude: $98^{\circ} 17^{\prime} \mathrm{E}$; eventDate: Aug. 24, 2005; recordedBy: Y. Ito; collectionID: C. Phengklai et al. 15067; institutionCode: BKF

c. country: Thailand; locality: Phu Kradung Natl Park. Tambon Srithan, Phu Kradung District; decimalLatitude: $16.90 \mathrm{~N}$; decimalLongitude: $101.80 \mathrm{E}$; eventDate: Nov. 1, 2002; recordedBy: Y. Ito; collectionID: A. prajaksood and S. Suddee 243; institutionCode: BKF

d. country: Thailand; locality: Tambon Putthawee, Makham district.; eventDate: Oct. 5, 2002; recordedBy: Y. Ito; collectionID: A. prajaksood and S. Suddee 171; institutionCode: BKF

e. country: Thailand; locality: Khao Yai Natl Park, Muang District.; decimalLatitude: $14^{\circ} 26^{\prime}$ 29" N; decimalLongitude: $101^{\circ} 22^{\prime} 11^{\prime \prime}$ E; eventDate: Sep. 28, 2002; recordedBy: Y. Ito; collectionID: A. prajaksood and S. Suddee 163; institutionCode: BKF

f. country: Thailand; locality: Sakon Nakhon; Phu Phan Natl Park.; verbatimLatitude: $16^{\circ} 51^{\prime}$ $\mathrm{N}$; verbatimLongitude: $103^{\circ} 55^{\prime} \mathrm{E}$; eventDate: Jul. 4, 1999; recordedBy: Y. Ito; collectionID: A. prajaksood 155; institutionCode: BKF

Distribution: Bangladesh, ?Cambodia, China (Southern), India (Western, Southern), Japan, ?Laos, ?Myanmar, Sri Lanka, Thailand, ?Vietnam.

\section{Order Ceratophyllales}

\section{Family Ceratophyllaceae}

\section{Genus Ceratophyllum L., 1753}

\section{Ceratophyllum demersum L., 1753}

\section{Materials}
a. country: Myanmar; verbatimLatitude: $16^{\circ} 53^{\prime} 19^{\prime \prime} \mathrm{N}$; verbatimLongitude: $95^{\circ} 52^{\prime} 22^{\prime \prime} \mathrm{E}$; recordedBy: Y. Ito; collectionID: TI040058; institutionCode: TI
b. country: Myanmar; verbatimLatitude: $16^{\circ} 53^{\prime} 19.18^{\prime \prime}$; verbatimLongitude: $95^{\circ} 52^{\prime} 28.59 "$; recordedBy: Y. Ito; collectionID: TI05152; institutionCode: TI
c. country: Myanmar; recordedBy: Y. Ito; collectionID: MBK080650; institutionCode: TI 
d. country: Thailand; locality: Kantchanabury; Hotel river Kwai; verbatimLatitude: $14^{\circ} 1^{\prime}$ 59" N; verbatimLongitude: $99^{\circ} 31^{\prime} 10^{\prime \prime}$ E; eventDate: Nov. 15, 2012; recordedBy: Y. Ito; collectionID: Y. Ito 1723; institutionCode: BKF

e. country: Thailand; locality: Bangkok; verbatimLatitude: $13^{\circ} 45^{\prime} \mathrm{N}$; verbatimLongitude: $100^{\circ} 30^{\prime}$ E; eventDate: Aug. 23, 1926; recordedBy: Y. Ito; collectionID: A. F. G. Kerr 11027; institutionCode: GH

f. country: Thailand; locality: Bangkok; verbatimLatitude: $13^{\circ} 45^{\prime} \mathrm{N}$; verbatimLongitude: $100^{\circ} 30^{\prime} \mathrm{E}$; eventDate: Aug. 23, 1926; recordedBy: Y. Ito; collectionID: A. Marcan 2135; institutionCode: $\mathrm{GH}$

Distribution: Worldwide.

\section{Order Proteales}

\section{Family Nelumbonaceae}

Genus Nelumbo Adans., 1763

Nelumbo nucifera Gaertn., 1788

Distribution: Native to tropics in Asia and Oceania.

\section{Order Saxifragales}

\section{Family Haloragaceae}

Genus Myriophyllum L., 1753

\section{Myriophyllum brasiliense Cambess, 1829}

Material

a. country: Thailand; locality: Pahayao Province; $6 \mathrm{~km}$ from Phayaoa; verbatimLatitude: $19^{\circ}$ 9' N; verbatimLongitude: $99^{\circ}$ 56' E; eventDate: Sep. 24, 1996; recordedBy: Y. Ito; collectionID: J.A.N. Parnell \& D.A. Simpson 1744; institutionCode: AAU

Distribution: Native in South America, naturalized in Asia, Oceania, and North America.

\section{Myriophyllum spicatum L., 1753}

\section{Material}

a. country: Myanmar; verbatimLatitude: $16^{\circ} 53^{\prime} 19^{\prime \prime} \mathrm{N}$; verbatimLongitude: $95^{\circ} 52^{\prime} 29^{\prime \prime} \mathrm{E}$; recordedBy: Y. Ito; institutionCode: TI

Distribution: China (nationwide), Japan, Myanmar; Europe. 


\section{Myriophyllum tetrandrum Roxb., 1820}

\section{Materials}

a. country: Thailand; locality: Rayong Province; Ban phe; verbatimLatitude: $12^{\circ} 40^{\prime} \mathrm{N}$; verbatimLongitude: $101^{\circ} 25^{\prime} \mathrm{E}$; eventDate: Dec. 16,1974 ; recordedBy: Y. Ito; collectionID: R. Geesink, P. Hiepko 7874; institutionCode: BKF

b. country: Thailand; locality: Chumphon, Ma Chana; verbatimLatitude: $10^{\circ} 0^{\prime} 24^{\prime \prime} \mathrm{N}$; verbatimLongitude: $99^{\circ}$ 3' 18" E; eventDate: Mar. 4, 1976; recordedBy: Y. Ito; collectionID: T. Gunlund 12127; institutionCode: BKF

c. country: Thailand; locality: ChaChoengsao Province; Khao Ang Rua Nai Wildlife Sanctuary; verbatimLatitude: $13^{\circ} 15^{\prime} \mathrm{N}$; verbatimLongitude: $101^{\circ} 50^{\prime} \mathrm{E}$; eventDate: Nov. 5, 1993; recordedBy: Y. Ito; collectionID: K. Larsen et al. 44234; institutionCode: AAU

d. country: Thailand; locality: Saraburi Province; Muang District, Sahm Lahn forest; verbatimLatitude: $14^{\circ} 31^{\prime} 51^{\prime \prime} \mathrm{N}$; verbatimLongitude: $100^{\circ} 54^{\prime} 34^{\prime \prime} \mathrm{E}$; eventDate: Nov. 23, 1974; recordedBy: Y. Ito; collectionID: J.F. Maxwell 74-995; institutionCode: AAU

e. country: Thailand; locality: Songkla Province; ca $4 \mathrm{~km}$ SE of Chana, along hwy 43; verbatimLatitude: $6^{\circ} 52^{\prime} 34^{\prime \prime} \mathrm{N}$; verbatimLongitude: $100^{\circ} 57^{\prime} 11$ " E; eventDate: Jul. 11 , 1985; recordedBy: Y. Ito; collectionID: J.F. Maxwell 85-702; institutionCode: AAU

f. country: Thailand; locality: Yala Province; verbatimLatitude: $6^{\circ} 34^{\prime} \mathrm{N}$; verbatimLongitude: 101 18' E; eventDate: Oct. 22, 1970; recordedBy: Y. Ito; collectionID: C. Charoenphol et al. 4144; institutionCode: AAU

Distribution: Bangladesh, China (Southern), India (nationwide), Malaysia (Peninsular), Myanmar, Thailand, Sri Lanka, ?Vietnam.

\section{Myriophyllum tuberculatum Roxb., 1820}

\section{Materials}

a. country: Myanmar; locality: Shan State; verbatimLatitude: $20^{\circ} 59^{\prime} 57^{\prime \prime} \mathrm{N}$; verbatimLongitude: $96^{\circ} 39^{\prime}$ 59" E; eventDate: Dec. 1, 2008; recordedBy: Y. Ito; collectionID: Tanaka et al. 080626; institutionCode: TI

b. country: Thailand; locality: Pattalung Province; Bah Baun District, Ban Naung Tong; verbatimLatitude: $7^{\circ} 13^{\prime} 34^{\prime \prime} \mathrm{N}$; verbatimLongitude: $100^{\circ} 8^{\prime} 39^{\prime \prime} \mathrm{E}$; eventDate: Jan. 12 , 1987; recordedBy: Y. Ito; collectionID: J.F. Maxwell 87-29; institutionCode: BKF

Distribution: Bangladesh, China (Southern), India (Eastern, Southern), Indonesia, Malaysia (Penninsular, Borneo), Myanmar, ?Pakistan, Thailand; Australia. 


\section{Order Fabales}

\section{Family Fabaceae}

Genus Neptunia Lour., 1790

Neptunia oleracea Lour., 1790

\section{Material}

a. country: Thailand; locality: Hotel river Kwai, Kantchanabury; verbatimLatitude: $14^{\circ} 1{ }^{\prime} 59^{\prime \prime}$ $\mathrm{N}$; verbatimLongitude: $99^{\circ} 31^{\prime} 10^{\prime \prime}$ E; eventDate: Nov. 15, 2012; recordedBy: Y. Ito; collectionID: Y. Ito 1720; institutionCode: BKF

Distribution: India, ?Myanmar, Thailand.

Notes: Fig. 8.

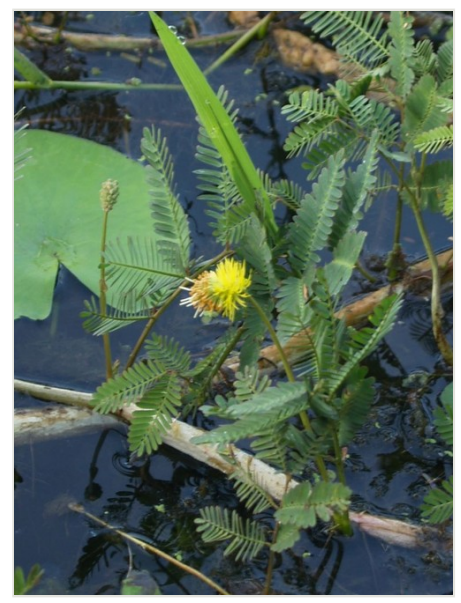

Figure 8.

Neptunia oleracea in Thailand (15 Nov 2012). Credit: Y. Ito. 


\section{Order Myrtales}

\section{Family Onagraceae}

Genus Ludwigia L., 1753

\section{Ludwigia adscendens (L.) H. Hara, 1953}

\section{Materials}

a. country: Thailand; locality: Hotel river Kwai, Kantchanabury; verbatimLatitude: $14^{\circ} 1^{\prime} 59^{\prime \prime}$ $\mathrm{N}$; verbatimLongitude: $99^{\circ} 31^{\prime} 10^{\prime \prime} \mathrm{E}$; eventDate: Nov. 15, 2012; recordedBy: Y. Ito; collectionID: Y. Ito 1720; institutionCode: BKF

b. country: Laos; locality: Savannaket Province; Nakai Plateau, Theun Douan lake, near Phong Sa Vahn resettlement village.; verbatimLatitude: $16^{\circ} 34^{\prime} 10^{\prime \prime} \mathrm{N}$; verbatimLongitude: 104 44' 54" E; eventDate: May. 4, 2007; recordedBy: Y. Ito; collectionID: J. F. Maxwell 07-313; institutionCode: $\mathrm{GH}$

C. country: Myanmar; verbatimLatitude: $16^{\circ} 53^{\prime} 19^{\prime \prime} \mathrm{N}$; verbatimLongitude: $95^{\circ} 52^{\prime} 29^{\prime \prime} \mathrm{E}$; eventDate: Dec. 8, 2006; recordedBy: Y. Ito; institutionCode: TI

d. country: Myanmar; verbatimLatitude: $16^{\circ} 53^{\prime} 19^{\prime \prime}$; verbatimLongitude: $95^{\circ} 52^{\prime} 28^{\prime \prime} \mathrm{E}$; eventDate: Dec. 1, 2008; recordedBy: Y. Ito; institutionCode: TI

e. country: Thailand; locality: Songkla Province, Talae Noi Waterfowl Reserve, $\mathrm{N}$ end of Lake Songkla, near Phattalung.; verbatimLatitude: $7^{\circ} 15^{\prime} \mathrm{N}$; verbatimLongitude: $100^{\circ} 26^{\prime}$ 16" E; eventDate: Dec. 28, 1978; recordedBy: Y. Ito; collectionID: G. Congdon \& C. Hamilton \#155; institutionCode: GH

f. country: Thailand; locality: Phetchabury.; verbatimLatitude: $13^{\circ} 24^{\prime} 30^{\prime \prime} \mathrm{N}$; verbatimLongitude: $99^{\circ} 48^{\prime} 44^{\prime \prime}$ E; eventDate: Nov. 14, 2012; recordedBy: Y. Ito; collectionID: Y. Ito 1709; institutionCode: BKF

Distribution: Bangladesh, Cambodia, China (nationwide), India (nationwide), Laos, Myanmar, Nepal, Sri Lanka, Thailand, Vietnam.

\section{Order Caryophyllales}

\section{Family Polygonaceae}

Genus Persicaria Mill., 1754

\section{Persicaria attenuata (R. Br.) Soják, 1974}

\section{Persicaria attenuata subsp. pulchra (Blume) K.L. Wilson, 1990}

\section{Material}

a. country: Thailand; locality: Bung Bonapet Nonhunting Area; verbatimLatitude: $15^{\circ} 41^{\prime} 40^{\prime \prime}$ $\mathrm{N}$; verbatimLongitude: $100^{\circ} 16^{\prime}$ 3" E; eventDate: Nov. 16, 2012; recordedBy: Y. Ito; collectionID: Y. Ito 1732; institutionCode: BKF 
Distribution: China (Southern [Taiwan]), India, Indonesia (Java, Sumatra), ?Japan, Malaysia (Borneo), ?Myanmar, Philippines, Thailand; ?Australia.

Notes: Fig. 9.

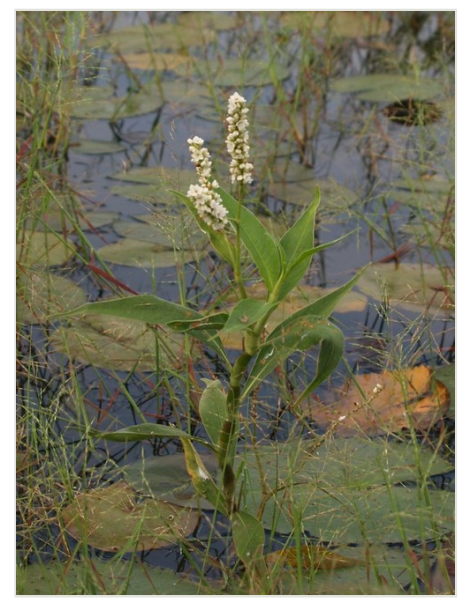

Figure 9.

Persicaria attenuata subsp. pulchra in Thailand (17 Nov 2012). Credit: Y. Ito.

\section{Order Solanales}

\section{Family Convolvulaceae}

\section{Genus Ipomoea L., 1753}

\section{Ipomoea aquatica Forssk., 1775}

\section{Materials}

a. country: Myanmar; verbatimLatitude: $16^{\circ} 53^{\prime} 19^{\prime \prime} \mathrm{N}$; verbatimLongitude: $95^{\circ} 52^{\prime} 29^{\prime \prime} \mathrm{E}$; recordedBy: Y. Ito; collectionID: ???; institutionCode: TI

b. country: Myanmar; locality: Mandalay Division; in fields, rice paddies etc., a serious weed "Kazun"; verbatimLatitude: $21^{\circ} 14^{\prime} 17^{\prime \prime}$ n; verbatimLongitude: $95^{\circ} 52^{\prime} 33$ " E; eventDate: Jan. 10, 1939; recordedBy: Y. Ito; collectionID: F.G. Dickason 9575; institutionCode: GH

c. country: Myanmar; locality: Kachin State; Bhamo district. Habitat Kaugyi; verbatimLatitude: $24^{\circ} 15^{\prime} 19^{\prime \prime} \mathrm{N}$; verbatimLongitude: $97^{\circ} 14^{\prime} 4^{\prime \prime} \mathrm{E}$; eventDate: Sep. 12 , 1908; recordedBy: Y. Ito; collectionID: -; institutionCode: GH

d. country: Thailand; locality: Songkla Prov., Ampoe Satingpra, Ban Satingpra; verbatimLatitude: $7^{\circ} 10^{\prime} 40^{\prime \prime} \mathrm{N}$; verbatimLongitude: $100^{\circ} 36^{\prime}$ 51" E; eventDate: Dec. 24 , 1978; recordedBy: Y. Ito; collectionID: G. Congdon \& C. Hamilton 127; institutionCode: $\mathrm{GH}$ 
e. country: Thailand; locality: Tarutao end of beach at Malacca Creek; verbatimLatitude: $6^{\circ}$ 33' 9" N; verbatimLongitude: $99^{\circ} 40^{\prime}$ 8" E; eventDate: Nov. 29, 1979; recordedBy: Y. Ito; collectionID: G. Congdon 207; institutionCode: $\mathrm{GH}$

f. country: Thailand; locality: Uttradit, Fishai, Bao Bak Klong; verbatimLatitude: $17^{\circ} 37^{\prime} 33^{\prime \prime}$ $\mathrm{N}$; verbatimLongitude: $100^{\circ}$ 6' 16" E; eventDate: Oct. 20, 1992; recordedBy: Y. Ito; collectionID: J. F. Maxwell 92-655; institutionCode: GH

g. country: Thailand; locality: Nakhon ratchasima Prov., Pak Chong Dist., Khao Yai Park; verbatimLatitude: $14^{\circ} 39^{\prime} 31^{\prime \prime} \mathrm{N}$; verbatimLongitude: $101^{\circ} 26^{\prime} \mathrm{E}$; eventDate: Oct. 17, 1985; recordedBy: Y. Ito; collectionID: G. Staples, T. Wongprassert 162; institutionCode: GH

h. country: Thailand; locality: Trang Prov., Yanta Khao Dist.; verbatimLatitude: $7^{\circ} 26^{\prime} 19^{\prime \prime} \mathrm{N}$; verbatimLongitude: $99^{\circ} 44^{\prime} 44^{\prime \prime}$ E; eventDate: Oct. 24, 1985; recordedBy: Y. Ito;

collectionID: G. Staples, T. Wongprassert 180; institutionCode: GH

i. country: Thailand; locality: Phuket Prov., near Nai Yang National Park; verbatimLatitude: $8^{\circ}$ 5' 19" N; verbatimLongitude: $98^{\circ} 17^{\prime}$ 52" E; eventDate: Oct. 25, 1985; recordedBy: Y. Ito; collectionID: G. Staples, T. Wongprassert 200; institutionCode: GH

j. country: Thailand; locality: Saraburi Prov.; verbatimLatitude: $14^{\circ} 32^{\prime} 56^{\prime \prime} \mathrm{N}$; verbatimLongitude: $100^{\circ} 54^{\prime} 13^{\prime \prime}$ E; eventDate: Apr. 11, 1985; recordedBy: Y. Ito; collectionID: G. Staples, T. Wongprassert 219; institutionCode: GH

k. country: Thailand; locality: Kanchanaburi Prov.; verbatimLatitude: $14^{\circ} 2^{\prime} 46^{\prime \prime} \mathrm{N}$; verbatimLongitude: $99^{\circ} 31^{\prime} 7^{\prime \prime}$ E; eventDate: Nov. 15, 1985; recordedBy: Y. Ito; collectionID: G. Staples, T. Wongprassert 274; institutionCode: GH

I. country: Thailand; locality: Kampangpet; verbatimLatitude: $7^{\circ} 11^{\prime} 31^{\prime \prime} \mathrm{N}$; verbatimLongitude: $100^{\circ} 35^{\prime} 35^{\prime \prime}$ E; eventDate: Nov. 28, 1977; recordedBy: Y. Ito; collectionID: C. Phengklai et al. 3944; institutionCode: $\mathrm{GH}$

m. country: Thailand; locality: Khon Kaen Prov., Chumphae Distr.; verbatimLatitude: $16^{\circ} 33^{\prime}$ 9" N; verbatimLongitude: $102^{\circ} 5^{\prime}$ 50" E; eventDate: May. 12, 1985; recordedBy: Y. Ito; collectionID: G. Staples, T. Wongprassert 399; institutionCode: GH

n. country: Thailand; locality: Songkla, Ranot; verbatimLatitude: $7^{\circ} 47^{\prime} 31^{\prime \prime} \mathrm{N}$; verbatimLongitude: $100^{\circ} 18^{\prime} 13^{\prime \prime}$ E; eventDate: Dec. 22, 2006; recordedBy: Y. Ito; collectionID: Pooma, R., Pattharahirantricin, N., Sirimongkol, S. 6548; institutionCode: GH

Distribution: Bangladesh, Cambodia, China (nationwide), India (nationwide), Laos, Myanmar, Nepal, Sri Lanka, Thailand, Vietnam.

\section{Family Plantaginaceae}

\section{Genus Callitriche L., 1753}

\section{Callitriche palustris L., 1753}

Distribution: Bangladesh, Bhutan, China (nationwide), India (Northern [Jammu and Kashmir], Southern [Tamil Nadu]), Japan, ?Myanmar, Nepal, Pakistan, Sri Lanka; Cosmopolitan. 
Genus Limnophila R. Br., 1810

\title{
Limnophila heterophylla (L.) Druce, 1914
}

\author{
Materials
}

a. country: Thailand; locality: Chiang Rai Province; Mae Chan District, Akcha village; verbatimLatitude: $20^{\circ} 12^{\prime} 4^{\prime \prime} \mathrm{N}$; verbatimLongitude: $99^{\circ} 56^{\prime} 45^{\prime \prime} \mathrm{E}$; eventDate: Nov. 7 , 1993; recordedBy: Y. Ito; collectionID: J.F. Maxwell 93-1364; institutionCode: GH

b. country: Thailand; locality: Chiang Rai Province; Maesai District, Wat Thamsaohin Phyanak; verbatimLatitude: $20^{\circ} 24^{\prime} 30 " \mathrm{~N}$; verbatimLongitude: $99^{\circ} 53^{\prime} \mathrm{E}$; eventDate: Feb. 14, 1983; recordedBy: Y. Ito; collectionID: H. Koyama, H. Terao, T. Wongprasert T-33500; institutionCode: $\mathrm{GH}$

c. country: Thailand; locality: Chiang Mai Province; Doi Inthanon, near guest house; verbatimLatitude: $18^{\circ} 32^{\prime} 55^{\prime \prime} \mathrm{N}$; verbatimLongitude: 98 31' 28" E; eventDate: Jul. 23, 1988; recordedBy: Y. Ito; collectionID: S. Tsugaru T-61736; institutionCode: GH

d. country: Thailand; locality: Saraburi; verbatimLatitude: $14^{\circ} 34^{\prime} 51^{\prime \prime} \mathrm{N}$; verbatimLongitude: $100^{\circ} 54^{\prime}$ 52" E; eventDate: Feb. 28, 1982; recordedBy: Y. Ito; collectionID: A. Ubolcholaket s.n.; institutionCode: AAU

e. country: Thailand; locality: Chonburi Province; Sattalip District, Taong Breng; verbatimLatitude: $12^{\circ} 43^{\prime} \mathrm{N}$; verbatimLongitude: $100^{\circ} 56^{\prime} \mathrm{E}$; eventDate: May. 7, 1972; recordedBy: Y. Ito; collectionID: J.F. Maxwell 72-221; institutionCode: AAU

f. country: Thailand; locality: Narathiwat Province; Toh Deang peat swamp; verbatimLatitude: $6^{\circ} 25^{\prime} 35^{\prime \prime} \mathrm{N}$; verbatimLongitude: 101 49' 26" E; eventDate: Aug. 28, 2001; recordedBy: Y. Ito; collectionID: C. Niyomdham 6533; institutionCode: BKF

Distribution: Cambodia, China (Eastern), India, Malaysia, ?Myanmar, Nepal, Sri Lanka, Thailand, Vietnam.

\section{Limnophila indica (Roxb.) Benth., 1835}

\section{Materials}

a. country: Myanmar; locality: Shan State; verbatimLatitude: $20^{\circ} 59^{\prime} 57^{\prime \prime} \mathrm{N}$; verbatimLongitude: $96^{\circ} 39^{\prime}$ 59" E; eventDate: Dec. 1, 2008; recordedBy: Y. Ito; collectionID: Tanaka et al. 080633; institutionCode: TI

b. country: Thailand; locality: Loei Province; Phu Kradung, S. of Loi; verbatimLatitude: $16^{\circ}$ 53' N; verbatimLongitude: $101^{\circ}$ 53' E; eventDate: Nov. 7, 1970; recordedBy: Y. Ito; collectionID: Ch. Charoenphol et al. 4797; institutionCode: AAU

c. country: Thailand; locality: Kanchanaburi Province; Thong Pha Phum District, Rintin Forest; verbatimLatitude: $14^{\circ} 47^{\prime} 41^{\prime \prime} \mathrm{N}$; verbatimLongitude: $98^{\circ} 44^{\prime} 42^{\prime \prime} \mathrm{E}$; eventDate: Nov. 6, 1979; recordedBy: Y. Ito; collectionID: T. Shimizu et al. T-21918; institutionCode: BKF

d. country: Thailand; locality: Mukdahan Province; Muang District, Dongman Village, along route 212; verbatimLatitude: $16^{\circ} 32^{\prime} 16^{\prime \prime} \mathrm{N}$; verbatimLongitude: $104^{\circ} 42^{\prime} 16^{\prime \prime} \mathrm{E}$; eventDate: Dec. 12, 1982; recordedBy: Y. Ito; collectionID: H. Koyama et al. T-30856; institutionCode: BKF

e. country: Thailand; locality: Khon Kaen Province; Phu Khieo Game reserve, $80 \mathrm{~km}$ E of Phetchabun; verbatimLatitude: $16^{\circ} 28^{\prime} 32^{\prime \prime} \mathrm{N}$; verbatimLongitude: $102^{\circ} 50^{\prime} 4^{\prime \prime} \mathrm{E}$; eventDate: Nov. 7, 1984; recordedBy: Y. Ito; collectionID: G. Murata T-41716; institutionCode: BKF 
Distribution: Bangladesh, ?Cambodia, China (South), India (nationwide), ?Indonesia, Japan, ?Laos, ?Malaysia, Myanmar, ?Nepal, Pakistan, Papua New Guinea, Sri Lanka, Thailand, ?Vietnam; Africa; Oceania.

\section{Limnophila sessiliflora (Vahl) Blume, 1826}

\section{Material}

a. country: Myanmar; locality: Kachin State; Tawang Hka (river) near Makaw Village, 6 miles $\mathrm{N}$ of Tanaing.; verbatimLatitude: $26^{\circ} 26^{\prime} 51^{\prime \prime} \mathrm{N}$; verbatimLongitude: $96^{\circ} 41^{\prime} 16^{\prime \prime} \mathrm{E}$; eventDate: Dec. 2, 2005; recordedBy: Y. Ito; collectionID: Murata et al. 040837; institutionCode: $\mathrm{TI}$

Distribution: Bangladesh, Bhutan, ?Cambodia, China (nationwide), India (nationwide), ?Indonesia (Java), Japan, ?Malaysia, Myanmar, Nepal, Sri Lanka, Thailand, ?Vietnam.

\section{Family Phrymaceae}

\section{Genus Mimulus L., 1753}

Mimulus orbicularis Wall. ex Benth., 1835

Material

a. country: Thailand; locality: Ayutthaya Provinve; Pathun Thani, Bangsit.; verbatimLatitude: 14ำ' 18' N; verbatimLongitude: $100^{\circ} 17^{\prime} 38^{\prime \prime}$ E; eventDate: Nov. 14, 1965; recordedBy: Y. Ito; collectionID: M. Tagawa \& K. Iwatsuki T-289; institutionCode: BKF, TI

Distribution: Bangladesh, India (Western [Orissa, West Bengal]), Myanmar, Thailand; Australia.

Notes: Barkera and Jobson (2013) reported a new locality of this species from northern Australia. 


\section{Order Lamiales}

\section{Family Lentibulariaceae}

\section{Genus Utricularia L., 1753}

\section{Utricularia aurea Lour., 1790}

\section{Materials}

a. country: Myanmar; locality: Inle Lake; verbatimLatitude: $20^{\circ} 35^{\prime} 21^{\prime \prime} \mathrm{N}$; verbatimLongitude: 96 54' 35" E; eventDate: Jan. 5, 1938; recordedBy: Y. Ito; collectionID: F.G. Dickason 7857; institutionCode: $\mathrm{GH}$

b. country: Myanmar; locality: Bago Division; Pyat Township.; verbatimLatitude: $18^{\circ} 42^{\prime} 22^{\prime \prime}$ N; verbatimLongitude: 95 5' 59" E; eventDate: Dec. 9, 2006; recordedBy: Y. Ito; collectionID: Sugawara et al. 036434; institutionCode: TI

c. country: Myanmar; locality: Kachin State; Tanaing Township; verbatimLatitude: $26^{\circ} 6^{\prime} 34^{\prime \prime}$ N; verbatimLongitude: $96^{\circ} 42^{\prime}$ 58" E; eventDate: Sep. 19, 2005; recordedBy: Y. Ito; collectionID: Tanaka et al. 040485; institutionCode: TI

d. country: Thailand; locality: Chanthaburi Province; Makham; verbatimLatitude: $13^{\circ} 0^{\prime} \mathrm{N}$; verbatimLongitude: $101^{\circ}$ 50' E; eventDate: Aug. 5, 1973; recordedBy: Y. Ito; collectionID: R. Geesink \& C. Phengkhlai 6333; institutionCode: AAU

e. country: Thailand; locality: Ayuthia Province; Sara Buri - Klang Dong; verbatimLatitude: $14^{\circ} 32^{\prime}$ 9" N; verbatimLongitude: $100^{\circ} 54^{\prime} 34^{\prime \prime} \mathrm{E}$; eventDate: Aug. 8, 1979; recordedBy: Y. Ito; collectionID: T. Shimizu, H. Toyokuni, H. Koyama, T. Yahara, C. Niyomdham 9479; institutionCode: $\mathrm{GH}$

f. country: Thailand; locality: Narathiwat Province; Swamp forest station, $\mathrm{N}$ of Sungai Kolok; verbatimLatitude: $6^{\circ} 4^{\prime} \mathrm{N}$; verbatimLongitude: $101^{\circ} 57^{\prime} \mathrm{E}$; eventDate: Aug. 17, 1995; recordedBy: Y. Ito; collectionID: K. Larsen et al. 45697; institutionCode: AAU

g. country: Thailand; locality: Saraburi; verbatimLatitude: $14^{\circ} 35^{\prime} 10^{\prime \prime} \mathrm{N}$; verbatimLongitude: $100^{\circ} 54^{\prime} 32 "$ E; eventDate: Feb. 28, 1982; recordedBy: Y. Ito; collectionID: A. Ubolcholaket s.n.; institutionCode: AAU

h. country: Thailand; locality: Naai Province; Tah Wang Pah District, Naha Han Village; verbatimLatitude: $19^{\circ} 66^{\prime} 44^{\prime \prime} \mathrm{N}$; verbatimLongitude: $100^{\circ} 45^{\prime}$ 16" E; eventDate: Mar. 15 , 2000; recordedBy: Y. Ito; collectionID: J.F. Maxwell 00-131; institutionCode: GH

i. country: Thailand; locality: Thonburi; verbatimLatitude: $13^{\circ} 45^{\prime} \mathrm{N}$; verbatimLongitude: $100^{\circ} 30^{\prime}$ E; eventDate: Nov. 15, 1970; recordedBy: Y. Ito; collectionID: J.F. Maxwell 70-85; institutionCode: AAU

j. country: Thailand; locality: Ang Thong Province; Muang District; verbatimLatitude: $14^{\circ} 35^{\prime}$ 41" N; verbatimLongitude: $100^{\circ} 27^{\prime} 25^{\prime \prime}$ E; eventDate: Dec. 11, 1971; recordedBy: Y. Ito; collectionID: J.F. Maxwell 71-787; institutionCode: AAU

k. country: Thailand; locality: Saraburi Province; Muang District, Sahm Lahn forest; verbatimLatitude: $14^{\circ} 31^{\prime} 51^{\prime \prime} \mathrm{N}$; verbatimLongitude: $100^{\circ} 54^{\prime} 34^{\prime \prime} \mathrm{E}$; eventDate: Jun. 30 , 1974; recordedBy: Y. Ito; collectionID: J.F. Maxwell 74-648; institutionCode: AAU

I. country: Thailand; locality: Songkla Province; Haad Yai District, Klong Hoy Kong, W of Toong Loong; verbatimLatitude: $7^{\circ} 0^{\prime} 13^{\prime \prime} \mathrm{N}$; verbatimLongitude: $100^{\circ} 27^{\prime} 25^{\prime \prime}$ e; eventDate: May. 30, 1985; recordedBy: Y. Ito; collectionID: J.F. Maxwell 85-548; institutionCode: $\mathrm{GH}$ 
m. country: Thailand; locality: Songkla Province; Muang - Chana Districts Border; verbatimLatitude: $6^{\circ} 59^{\prime} 6^{\prime \prime} \mathrm{N}$; verbatimLongitude: $100^{\circ} 39^{\prime} 26^{\prime \prime}$ e; eventDate: Jan. 17 , 1985; recordedBy: Y. Ito; collectionID: J.F. Maxwell 85-75; institutionCode: GH

n. country: Thailand; locality: Surattani Province; Ban Takun Distr., Klong Saeng Wildlife Sanctuary.; verbatimLatitude: $9^{\circ}$ 9' 43" N; verbatimLongitude: $98^{\circ} 53^{\prime} 21^{\prime \prime} \mathrm{E}$; eventDate: Feb. 16, 1994; recordedBy: Y. Ito; collectionID: J.F. Maxwell 94-230; institutionCode: GH

o. country: Thailand; locality: $\mathrm{N}$ of pie Mai; eventDate: Mar. 19, 1958; recordedBy: Y. Ito; collectionID: Th. Sorensen 2145; institutionCode: AAU

p. country: Thailand; locality: Chaiyaphum Province; Thung Kra Mang; verbatimLatitude: $16^{\circ}$ $15^{\prime} \mathrm{N}$; verbatimLongitude: $101^{\circ} 30^{\prime} \mathrm{E}$; eventDate: Aug. 9, 1972; recordedBy: Y. Ito; collectionID: K. Larsen et al. 31632; institutionCode: AAU

q. country: Thailand; locality: Chanthaburi Province; Bor Rai50 km E of Makham; verbatimLatitude: $12^{\circ} 52^{\prime} \mathrm{N}$; verbatimLongitude: $102^{\circ} 7^{\prime} \mathrm{E}$; eventDate: Sep. 1, 1972; recordedBy: Y. Ito; collectionID: K. Larsen et al. 32313; institutionCode: AAU

r. country: Thailand; locality: Narathiwat Province; S of Naratiwat; verbatimLatitude: $6^{\circ} 30^{\prime}$ $\mathrm{N}$; verbatimLongitude: $101^{\circ} 45^{\prime} \mathrm{E}$; eventDate: Mar. 9, 1974; recordedBy: Y. Ito; collectionID: K. Larsen et al. 33129; institutionCode: AAU

s. country: Thailand; locality: Nakhon Ratchasima Province; Pak Thong Chai District, E part of Khao Yai Natl Park; verbatimLatitude: $14^{\circ} 22^{\prime} \mathrm{N}$; verbatimLongitude: $101^{\circ} 12^{\prime} \mathrm{E}$; eventDate: Aug. 11, 1968; recordedBy: Y. Ito; collectionID: K. Larsen et al. 3339; institutionCode: AAU

t. country: Thailand; locality: Nakhon Ratchasima Province; Phimai; verbatimLatitude: $15^{\circ} 5^{\prime}$ $\mathrm{N}$; verbatimLongitude: $101^{\circ} 39^{\prime} \mathrm{E}$; eventDate: Jan. 12, 1967; recordedBy: Y. Ito; collectionID: T. Smitinand 33646; institutionCode: AAU

u. country: Thailand; locality: Chiang Mai Province; San Sai District; verbatimLatitude: $18^{\circ}$ 56' 29" N; verbatimLongitude: $99^{\circ}$ 1' 48" E; eventDate: Sep. 16, 1958; recordedBy: Y. Ito; collectionID: Th. Sorensen 5017; institutionCode: AAU

v. country: Thailand; locality: Narathiwat Province; Kok Dam peat Swamp forest; verbatimLatitude: $6^{\circ} 26^{\prime} 17^{\prime \prime} \mathrm{N}$; verbatimLongitude: $101^{\circ} 49^{\prime} 26^{\prime \prime} \mathrm{E}$; eventDate: Feb. 29 , 1984; recordedBy: Y. Ito; collectionID: C. Niyomdham 807; institutionCode: AAU

w. country: Thailand; locality: Chainat Province; ca. 10 miles W of ta Klee toward; verbatimLatitude: $15^{\circ} 14^{\prime} \mathrm{N}$; verbatimLongitude: $100^{\circ} 15^{\prime} \mathrm{E}$; eventDate: Nov. 28, 1959; recordedBy: Y. Ito; collectionID: L. B. \& E. C. Abbe, T. Smitinand 9232; institutionCode: $\mathrm{GH}$

X. country: Thailand; locality: Buri Ram Province; near Ban Kruat; verbatimLatitude: $14^{\circ} 30^{\prime}$ $\mathrm{N}$; verbatimLongitude: $103^{\circ} 7^{\prime} \mathrm{E}$; eventDate: Oct. 4, 1984; recordedBy: Y. Ito; collectionID: G. Murata, C. Phengklai, S. Mitsuta, H. Nagamasu, N. Nantasan T-37406; institutionCode: AAU

y. country: Thailand; locality: Khao San Yot Natl Park.; verbatimLatitude: $12^{\circ} 14$ ' 40" N; verbatimLongitude: $99^{\circ} 55^{\prime}$ 58" E; eventDate: Nov. 13, 2012; recordedBy: Y. Ito; collectionID: Y. Ito 1702; institutionCode: BKF

z. country: Thailand; locality: Phetchabury.; verbatimLatitude: $13^{\circ} 30^{\prime} 6^{\prime \prime} \mathrm{N}$; verbatimLongitude: $99^{\circ}$ 47' 37" E; eventDate: Nov. 14, 2012; recordedBy: Y. Ito; collectionID: Y. Ito 1712; institutionCode: BKF

aa. country: Thailand; locality: Kantchanabury; verbatimLatitude: $14^{\circ} 39^{\prime} 21^{\prime \prime} \mathrm{N}$; verbatimLongitude: $98^{\circ} 42^{\prime} 27^{\prime \prime}$ E; eventDate: Nov. 15, 2012; recordedBy: Y. Ito; collectionID: Y. Ito 1730; institutionCode: BKF

Distribution: Bangladesh, China (Central, South), India (nationwide), Japan, Malesia (throughout), Nepal, Pakistan; Oceania. 


\section{Utricularia australis $\mathrm{R}$. Br., 1810}

\section{Materials}

a. country: Myanmar; locality: Bago Division, Donese; verbatimLatitude: $17^{\circ} 37^{\prime} 6^{\prime \prime} \mathrm{N}$; verbatimLongitude: $95^{\circ} 47^{\prime}$ 22" E; eventDate: Dec. 8, 2006; recordedBy: Y. Ito; collectionID: Sugawara et al. 036321; institutionCode: TI

b. country: Myanmar; locality: Shan State; Pindaya Township, Inya lake; verbatimLatitude: $20^{\circ} 59^{\prime} 57^{\prime \prime} \mathrm{N}$; verbatimLongitude: $96^{\circ} 39^{\prime} 59^{\prime \prime} \mathrm{E}$; eventDate: Dec. 1, 2008; recordedBy: Y. Ito; collectionID: Tanaka et al. 080627; institutionCode: TI

c. country: Myanmar; locality: Shan State; Pindaya Township, Inlay lake; verbatimLatitude: $20^{\circ} 32$ ' 2" N; verbatimLongitude: $96^{\circ}$ 53' 53" E; eventDate: Dec. 3, 2008; recordedBy: Y. Ito; collectionID: Tanaka et al. 080644; institutionCode: TI

d. country: Myanmar; locality: Shan State; Nyaung Shwe Towhship, Inlay Lake; verbatimLatitude: $20^{\circ} 32^{\prime}$ 2" N; verbatimLongitude: $96^{\circ} 53^{\prime} 53^{\prime \prime} \mathrm{E}$; eventDate: Dec. 3 , 2008; recordedBy: Y. Ito; collectionID: Tanaka et al. 080659; institutionCode: TI

e. country: Myanmar; locality: Mandaley Region; Maymyo (Pyinoolwin) Plateau; verbatimLatitude: $22^{\circ} 2^{\prime} 12^{\prime \prime} \mathrm{N}$; verbatimLongitude: $96^{\circ} 28^{\prime} 10^{\prime \prime} \mathrm{E}$; eventDate: Jan. 9 , 1912; recordedBy: Y. Ito; collectionID: J.H. Lace 5948; institutionCode: GH

f. country: Myanmar; locality: Mandaley Region; Maymyo (Pyinoolwin); verbatimLatitude: $22^{\circ} 2^{\prime} 12^{\prime \prime} \mathrm{N}$; verbatimLongitude: $96^{\circ} 28^{\prime} 10^{\prime \prime} \mathrm{E}$; eventDate: Jan. 4, 1933; recordedBy: Y. Ito; collectionID: F.G. Dickason 5994; institutionCode: GH

Distribution: Bhutan, China (nationwide), India (nationwide), Indonesia (Java, Sumatra), Japan, Nepal, Pakistan, Papua New Guinea, Philippines, Sri Lanka; Africa, Europe, Oceania.

\section{Utricularia gibba L., 1753}

\section{Materials}

a. country: Myanmar; locality: Shan State; Inlay lake, Nyanug She Township; verbatimLatitude: $16^{\circ} 53^{\prime} 19^{\prime \prime} \mathrm{N}$; verbatimLongitude: $95^{\circ} 52^{\prime} 29^{\prime \prime} \mathrm{E}$; eventDate: Dec. 3 , 2008; recordedBy: Y. Ito; collectionID: Tanaka et al. 080639; institutionCode: TI

b. country: Myanmar; locality: Tenasserim Division, Tawer District, Yebyu Township, Kan Bank Village area, coastal region; verbatimLatitude: $12^{\circ} 5^{\prime} 11^{\prime \prime} \mathrm{N}$; verbatimLongitude: $99^{\circ}$ 0' 51" E; eventDate: Mar. 2, 1996; recordedBy: Y. Ito; collectionID: J.F. Maxwell 96-335; institutionCode: BKF

C. country: Thailand; locality: Songkla Province; Talae Noi waterfowl reserve; verbatimLatitude: $7^{\circ} 15^{\prime} \mathrm{N}$; verbatimLongitude: $100^{\circ} 26^{\prime} 16$ " E; eventDate: Dec. 28, 1978; recordedBy: Y. Ito; collectionID: G. Congdon, C. Hamilton 159; institutionCode: AAU

d. country: Thailand; locality: Lopburi Province; Supcham Pa Hill; verbatimLatitude: $14^{\circ} 50^{\prime}$ $\mathrm{N}$; verbatimLongitude: $100^{\circ} 55^{\prime} \mathrm{E}$; eventDate: Nov. 19, 1984; recordedBy: Y. Ito; collectionID: G. Murata, C. Phengklai, S. Mitsuta, T. Yahara, H. Nagamasu, N. Nantasan T-50927; institutionCode: BKF

e. country: Thailand; locality: Chiang Mai Province; Doi Inthanon, near guest house; verbatimLatitude: $18^{\circ} 32^{\prime} 55^{\prime \prime} \mathrm{N}$; verbatimLongitude: $98^{\circ} 31^{\prime} 29^{\prime \prime} \mathrm{E}$; eventDate: Jul. 23 , 1988; recordedBy: Y. Ito; collectionID: S. Tsugaru T-61738; institutionCode: BKF

f. country: Thailand; locality: Thathungna Dam, Kantchanabury; verbatimLatitude: $14^{\circ} 13^{\prime}$ 53" N; verbatimLongitude: $99^{\circ} 13^{\prime} 44^{\prime \prime}$ E; eventDate: Nov. 15, 2012; recordedBy: Y. Ito; collectionID: Y. Ito 1725; institutionCode: BKF 
Distribution: Bangladesh, China (nationwide), India (nationwide), Indonesia (nationwide), Japan, Malaysia (nationwide), Myanmar, Nepal, Papua New Guinea, Sri Lanka, Thailand, ?Vietnam; Africa; Oceania; N. America; S. America.

\section{Utricularia punctata Wall. ex A. DC., 1844}

\section{Materials}

a. country: Myanmar; locality: Tenasserim Division, Tawer District, Yebyu Township, Kan Bank Village area, coastal region; verbatimLatitude: $12^{\circ} 5^{\prime} 11^{\prime \prime} \mathrm{N}$; verbatimLongitude: $99^{\circ}$ 0' 51" E; eventDate: Mar. 2, 1996; recordedBy: Y. Ito; collectionID: J.F. Maxwell 96-336; institutionCode: BKF

b. country: Thailand; locality: Phang-nga Province; Kuraburi District, Bangwan Stream; verbatimLatitude: $9^{\circ} 13^{\prime} 34^{\prime \prime} \mathrm{N}$; verbatimLongitude: $98^{\circ} 26^{\prime} 22^{\prime \prime} \mathrm{E}$; eventDate: Dec. 28 , 2006; recordedBy: Y. Ito; collectionID: T. Muadsud 210; institutionCode: BKF

c. country: Thailand; locality: Narathiwat Province; Bang Nara River; verbatimLatitude: $6^{\circ}$ 23' 20" N; verbatimLongitude: $101^{\circ} 49^{\prime} 26 "$ E; eventDate: Jan. 20, 2000; recordedBy: Y. Ito; collectionID: C. Niyomdham et al. 5990; institutionCode: BKF

Distribution: China (South), Indonesia (Sumatra), Malaysia (Borneo, Malaya), Myanmar, Thailand, ?Vietnam.

\section{Utricularia stellaris L. f., 1781}

\section{Materials}

a. country: Myanmar; locality: Rangoon; verbatimLatitude: $16^{\circ} 49^{\prime} 37^{\prime \prime} \mathrm{N}$; verbatimLongitude: 96 $8^{\prime}$ 58" E; eventDate: Jan. 2, 1938; recordedBy: Y. Ito; collectionID: F.G. Dickason 6965; institutionCode: GH

b. country: Myanmar; locality: Bago Division; Pyat Township.; verbatimLatitude: $18^{\circ} 49^{\prime} 44^{\prime \prime}$ N; verbatimLongitude: 95 18' 6" E; eventDate: Dec. 7, 2008; recordedBy: Y. Ito; collectionID: Tanaka et al. 080765; institutionCode: TI

Distribution: Bangladesh, India (nationwide), Myanmar, Nepal, Sri lanka; Africa.

Notes: Fig. 10. 


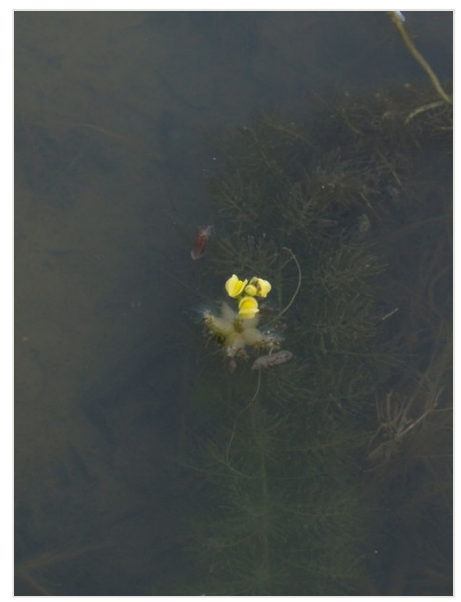

Figure 10.

Utricularia stellaris in Myanmar (7 Dec 2008). Credit: Y. Ito.

\section{Order Asterales}

\section{Family Menyanthaceae}

\section{Genus Nymphoides Ség., 1754}

\section{Nymphoides aurantiaca (Dalzell) Kuntze, 1891}

\section{Materials}

a. country: Thailand; locality: Krabi province; Ban Sai Khao, Konglom; verbatimLatitude: $7^{\circ}$ 44' 59" N; verbatimLongitude: $99^{\circ} 15^{\prime} 48^{\prime \prime} \mathrm{E}$; eventDate: Oct. 1, 1960; recordedBy: Y. Ito; collectionID: ???; institutionCode: TI

b. country: Thailand; locality: Surat Thani Province; Tochang District, Ban Phace, Elong Sai Subdistrict; verbatimLatitude: $9^{\circ} 18^{\prime} 15^{\prime \prime} \mathrm{N}$; verbatimLongitude: $99^{\circ} 3^{\prime} 19^{\prime \prime} \mathrm{E}$; eventDate: Feb. 5, 1987; recordedBy: Y. Ito; collectionID: E.P. 1246; institutionCode: BKF

C. country: Thailand; locality: Chanthabury Province; $18 \mathrm{~km} \mathrm{~W}$ of Trak; verbatimLatitude: $12^{\circ}$ $57^{\prime} \mathrm{N}$; verbatimLongitude: $102^{\circ} 0^{\prime} \mathrm{E}$; eventDate: Oct. 23,1972 ; recordedBy: Y. Ito; collectionID: No. 2027; institutionCode: BKF

d. country: Thailand; locality: Surin; verbatimLatitude: $14^{\circ} 55^{\prime} 53^{\prime \prime} \mathrm{N}$; verbatimLongitude: $103^{\circ} 29^{\prime} 17^{\prime \prime}$ E; eventDate: Nov. 20, 1976; recordedBy: Y. Ito; collectionID: J.F. Maxwell 72-531; institutionCode: AAU

e. country: Thailand; locality: Ranong Province; Ban phe; verbatimLatitude: $12^{\circ} 40^{\prime} \mathrm{N}$; verbatimLongitude: $101^{\circ} 25^{\prime} \mathrm{E}$; eventDate: Dec. 16,1974 ; recordedBy: Y. Ito; collectionID: C. Phengklai et al. 3303; institutionCode: BKF, GH

f. country: Thailand; locality: Ranong Province; near the town; verbatimLatitude: $9^{\circ} 57^{\prime} 14^{\prime \prime}$ N; verbatimLongitude: $98^{\circ} 36^{\prime} 22^{\prime \prime}$ E; eventDate: Nov. 23, 1970; recordedBy: Y. Ito; collectionID: R. Geesink, P. Hiepko 7871; institutionCode: BKF 
g. country: Thailand; locality: Surattani Province; Tachang Ban Tahsae District, Klong Sai sub-district; verbatimLatitude: $9^{\circ} 17^{\prime} 58^{\prime \prime} \mathrm{N}$; verbatimLongitude: $99^{\circ} 3^{\prime} 27^{\prime \prime} \mathrm{E}$; eventDate: Feb. 5, 1987; recordedBy: Y. Ito; collectionID: T. Smitinand et al. 11591; institutionCode: BKF

Distribution: India (Southern), Papua New Guinea, Thailand; Oceania.

Notes: Fig. 11.

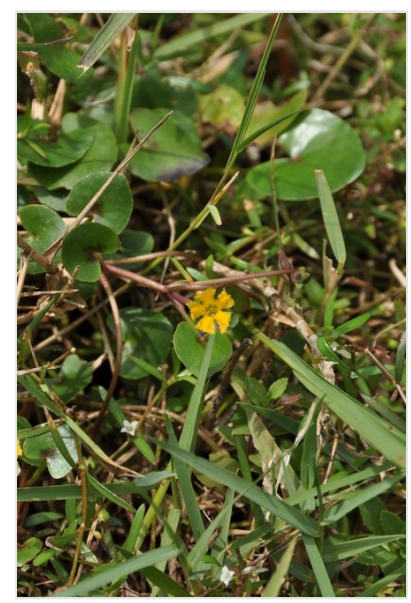

Figure 11.

Nymphoides aurantiaca in Thailand (15 May 2000). Credit: R. Pooma.

\section{Nymphoides cambodiana (Hance) Tippery, 2009}

Materials

a. country: Thailand; locality: Roi-et Province; verbatimLatitude: $16^{\circ} 2^{\prime} \mathrm{N}$; verbatimLongitude: $103^{\circ} 38^{\prime} \mathrm{E}$; eventDate: Jun. 22, 1969; recordedBy: Y. Ito; collectionID: J.F. Maxwell 87-165; institutionCode: GH

b. country: Thailand; locality: Nakhon Ratchasima Province; verbatimLatitude: $14^{\circ} 36^{\prime} \mathrm{N}$; verbatimLongitude: $101^{\circ} 33^{\prime} \mathrm{E}$; eventDate: Feb. 11, 1975; recordedBy: Y. Ito; collectionID: No. 46634; institutionCode: BKF

Distribution: Cambodia, Laos, Thailand, Vietnam.

\section{Nymphoides hydrophylla (Lour.) Kuntze, 1891}

\section{Materials}

a. country: Myanmar; locality: Shan State; Inlay lake, Nyanug She Township; verbatimLatitude: $20^{\circ} 32^{\prime}$ 2" N; verbatimLongitude: $96^{\circ} 53^{\prime} 53^{\prime \prime}$ E; eventDate: Dec. 3 , 2008; recordedBy: Y. Ito; collectionID: A. Ubolcholaket s.n.; institutionCode: AAU 
b. country: Myanmar; locality: Kachin State; S of Tanaing, Hukaung Valley; verbatimLatitude: $26^{\circ} 06^{\prime} 34 " \mathrm{~N}$; verbatimLongitude: $96^{\circ} 42^{\prime}$ 58" E; eventDate: Sep. 19, 2005; recordedBy: Y. Ito; collectionID: Nb. Tanaka et al. 080654; institutionCode: TI

c. country: Thailand; locality: Songkhla Province; Songkhla - Pattani road, ca $50 \mathrm{~km}$ from Songkhla; verbatimLatitude: $6^{\circ} 53^{\prime} \mathrm{N}$; verbatimLongitude: $100^{\circ} 55^{\prime} \mathrm{E}$; eventDate: Oct. 31 , 1990; recordedBy: Y. Ito; collectionID: Nb. Tanaka et al. 040480; institutionCode: TI

d. country: Thailand; locality: Chanthabury Province; between Chanthaburi and Trat; verbatimLatitude: $12^{\circ} 40^{\prime} \mathrm{N}$; verbatimLongitude: $102^{\circ} 20^{\prime} \mathrm{E}$; eventDate: Sep. 3, 1972; recordedBy: Y. Ito; collectionID: K. Larsen et al. 41006; institutionCode: AAU

e. country: Thailand; locality: Loei Province; Khunnaamphong, Phukradueng Natl Park; verbatimLatitude: $16^{\circ} 54^{\prime} \mathrm{N}$; verbatimLongitude: $101^{\circ} 45^{\prime} \mathrm{E}$; eventDate: Sep. 8, 1988; recordedBy: Y. Ito; collectionID: K. Larsen et al. 32398; institutionCode: AAU

f. country: Thailand; locality: Narathiwat; verbatimLatitude: $6^{\circ} 26^{\prime} \mathrm{N}$; verbatimLongitude: $101^{\circ} 49^{\prime}$ E; eventDate: Sep. 18, 1965; recordedBy: Y. Ito; collectionID: R. Pooma 85; institutionCode: BKF

g. country: Thailand; locality: Ranong Province; Bang Ben, Laem Son Forest Park; verbatimLatitude: $9^{\circ} 28^{\prime} \mathrm{N}$; verbatimLongitude: $98^{\circ} 23^{\prime}$ E; eventDate: Sep. 10, 1982; recordedBy: Y. Ito; collectionID: Chamlong 1104; institutionCode: BKF

Distribution: Cambodia, China (Southern [Taiwan]), Laos, Myanmar, Thailand, Vietnam.

Notes: Fig. 12.

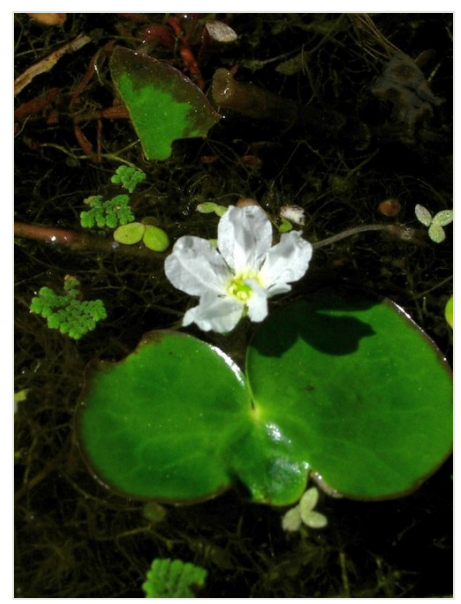

Figure 12.

Nymphoides hydrophylla in Myanmar (3 Dec 2008). Credit: Y. Ito.

\section{Nymphoides indica (L.) Kuntze, 1891}

\section{Materials}

a. country: Myanmar; locality: Bago Division; W of Pyay; verbatimLatitude: $18^{\circ} 42^{\prime} 22^{\prime \prime} \mathrm{N}$; verbatimLongitude: $95^{\circ}$ 5' 59" E; eventDate: Dec. 9, 2006; recordedBy: Y. Ito; collectionID: T. Shimizu et al. T-29263; institutionCode: BKF 
b. country: Myanmar; locality: Rakhain State; Linthar, Ngapali Dam; verbatimLatitude: $19^{\circ}$ 24' 27" N; verbatimLongitude: $94^{\circ} 20^{\prime}$ 3" E; eventDate: Dec. 12, 2006; recordedBy: Y. Ito; collectionID: T. Sugawara et al. 036430; institutionCode: TI

c. country: Myanmar; locality: Shan State; Inya lake, N of Pindaya; verbatimLatitude: $20^{\circ} 59^{\prime}$ $57^{\prime \prime} \mathrm{N}$; verbatimLongitude: $96^{\circ} 39^{\prime}$ 59" E; eventDate: Dec. 1, 2008; recordedBy: Y. Ito; collectionID: T. Sugawara et al. 036591; institutionCode: TI

d. country: Myanmar; locality: Shan State; Inlaylake, Nyaung Shwe Township; verbatimLatitude: $20^{\circ} 32^{\prime} 2^{\prime \prime} \mathrm{N}$; verbatimLongitude: $96^{\circ} 53^{\prime} 53^{\prime \prime} \mathrm{E}$; eventDate: Dec. 3 , 2008; recordedBy: Y. Ito; collectionID: Nb. Tanaka et al. 080629; institutionCode: TI

e. country: Thailand; locality: Chiang Mai Province; Northern District, along road to San Kamphaeng, E of Chiang Mai; verbatimLatitude: $18^{\circ} 47^{\prime} 16^{\prime \prime} \mathrm{N}$; verbatimLongitude: $99^{\circ} 0^{\prime}$ 39" E; eventDate: Jun. 17, 1968; recordedBy: Y. Ito; collectionID: Nb. Tanaka et al. 080643; institutionCode: TI

f. country: Thailand; locality: Chanthabury Province; $18 \mathrm{~km} \mathrm{~W}$ of Trak; verbatimLatitude: $12^{\circ}$ $57^{\prime} \mathrm{N}$; verbatimLongitude: $102^{\circ}$ 0' E; eventDate: Oct. 2, 1972; recordedBy: Y. Ito; collectionID: C.F. van Beusekom, C. Phengkhlai 1302; institutionCode: AAU

g. country: Thailand; locality: Songkla Province; Muang - Chana Districts Border; verbatimLatitude: $6^{\circ} 59^{\prime}$ 6" N; verbatimLongitude: $100^{\circ} 39^{\prime} 26$ " E; eventDate: Jan. 17 , 1985; recordedBy: Y. Ito; collectionID: J.F. Maxwell 72-532; institutionCode: AAU

h. country: Thailand; locality: Songla Province; Talae Noi Waterfowl Reserve, $\mathrm{N}$ end of Lake Songkla, near Phattalung; verbatimLatitude: $7^{\circ} 15^{\prime} \mathrm{N}$; verbatimLongitude: $100^{\circ} 26^{\prime} 16^{\prime \prime} \mathrm{E}$; eventDate: Dec. 28, 1978; recordedBy: Y. Ito; collectionID: J.F. Maxwell 85-74; institutionCode: $\mathrm{GH}$

i. country: Thailand; locality: Narathiwat Province; Paa Waai, Su Ngi Paadee; verbatimLatitude: $6^{\circ} 7^{\prime} 37^{\prime \prime} \mathrm{N}$; verbatimLongitude: $101^{\circ} 54^{\prime}$ 52" E; eventDate: Sep. 4 , 1987; recordedBy: Y. Ito; collectionID: G. Congdon, C. Hamilton 153; institutionCode: AAU

j. country: Thailand; locality: Surin; verbatimLatitude: $14^{\circ} 55^{\prime} 53^{\prime \prime} \mathrm{N}$; verbatimLongitude: $103^{\circ} 29^{\prime} 17^{\prime \prime}$ E; eventDate: Nov. 20, 1976; recordedBy: Y. Ito; collectionID: C. Niyomdham \& D. Sriboonma 1544; institutionCode: AAU

k. country: Thailand; locality: Nakhon Nayok Province; verbatimLatitude: $14^{\circ} 12^{\prime} 17^{\prime \prime} \mathrm{N}$; verbatimLongitude: $101^{\circ} 13^{\prime} 36 " \mathrm{E}$; eventDate: Aug. 6, 1970; recordedBy: Y. Ito; collectionID: C. Phengklai et al. 3304; institutionCode: GH

I. country: Thailand; locality: Phitsanulok Province; Tung Salaeng Luang; verbatimLatitude: $16^{\circ} 59^{\prime} \mathrm{N}$; verbatimLongitude: $100^{\circ} 53^{\prime} \mathrm{E}$; eventDate: Jul. 19, 1966; recordedBy: Y. Ito; collectionID: C. Phengklai et al. 3750; institutionCode: GH

m. country: Thailand; locality: East, Si Saket Prov.; verbatimLatitude: $15^{\circ} 7^{\prime} \mathrm{N}$; verbatimLongitude: $104^{\circ} 15^{\prime} \mathrm{E}$; eventDate: Oct. 7, 1984; recordedBy: Y. Ito; collectionID: K. Larsen et al. 512; institutionCode: AAU

n. country: Thailand; locality: Kantchanabury Province; Hotel river Kwai; verbatimLatitude: 14ำ 1' 59" N; verbatimLongitude: $99^{\circ}$ 31' 10" E; eventDate: Nov. 15, 2012; recordedBy: Y. Ito; collectionID: G. Murata, C. Phengklai, S. Mitsuta, H. Nagamasu, N. Nantasan T-49919; institutionCode: AAU, GH

o. country: Thailand; locality: Surin; verbatimLatitude: $14^{\circ} 55^{\prime} 53^{\prime \prime} \mathrm{N}$; verbatimLongitude: $103^{\circ} 29^{\prime} 17$ " E; eventDate: Dec. 8, 1976; recordedBy: Y. Ito; collectionID: Y. Ito 1718; institutionCode: BKF

Distribution: Bangladesh, ?Cambodia, China, India (nationwide), ?Indonesia, Japan, ? Malaysia, Myanmar, Nepal, Papua New Guinea, Sri Lanka, ?Vietnam; Oceania; N. America; S. America. 


\section{Analysis}

The 78 species were classified into six geographic categories: Widespread; Eurasian cooltemperate; Asia - Australia; Tropical Asia; Pan-Tropics; invasive (Table 2). The data concerning the general distribution was taken Boyce (2012), Boyce et al. (2012), Chayamarit (2005), Cook (1996), Guo et al. (2010), Haynes (2001f), Haynes (2001d), Haynes (2001a), Haynes (2001e), Haynes (2001b), Haynes (2001c), Ho and Ornduff (1995), Ito (2013b), Ito et al. (2009), Landolt (2001), Simpson (2008), Larsen (2000), Tippery et al. (2009), Yamazaki (1990), Barkera and Jobson (2013).

Table 2.

A classification of aquatic plants in Myanmar and Thailand in distribution.

\begin{tabular}{|c|c|c|c|c|c|}
\hline Widespread & $\begin{array}{l}\text { Eurasian cool- } \\
\text { temperate }\end{array}$ & Asia - Australia & Asian tropics & Pan-Tropics & Invasive \\
\hline Acorus calamus & $\begin{array}{l}\text { Alisma plantago- } \\
\text { aquatica }\end{array}$ & Acorus gramineus & $\begin{array}{l}\text { Aponogeton } \\
\text { lakhonensis }\end{array}$ & $\begin{array}{l}\text { Hydrilla } \\
\text { verticillata }\end{array}$ & $\begin{array}{l}\text { Cabomba } \\
\text { caroliniana }\end{array}$ \\
\hline $\begin{array}{l}\text { Caldesia } \\
\text { parnassifolia }\end{array}$ & $\begin{array}{l}\text { Potamogeton } \\
\text { lucens }\end{array}$ & Blyxa aubertii & $\begin{array}{l}\text { Barclaya } \\
\text { longifolia }\end{array}$ & $\begin{array}{l}\text { Ipomoea } \\
\text { aquatica }\end{array}$ & Egeria densa \\
\hline $\begin{array}{l}\text { Callitriche } \\
\text { stagnalis }\end{array}$ & $\begin{array}{l}\text { Vallisneria } \\
\text { spiralis }\end{array}$ & $\begin{array}{l}\text { Blyxa } \\
\text { echinosperma }\end{array}$ & $\begin{array}{l}\text { Blyxa } \\
\text { quadricostata }\end{array}$ & $\begin{array}{l}\text { Lemna } \\
\text { aequinoctialis }\end{array}$ & $\begin{array}{l}\text { Eichhornia } \\
\text { crassipes }\end{array}$ \\
\hline $\begin{array}{l}\text { Ceratophyllum } \\
\text { demersum }\end{array}$ & & Blyxa japonica & Crinum thaianum & $\begin{array}{l}\text { Ludwigia } \\
\text { adscendens }\end{array}$ & Elodea nutalli \\
\hline $\begin{array}{l}\text { Landoltia } \\
\text { punctata }\end{array}$ & & Hydrocharis dubia & $\begin{array}{l}\text { Cryptocoryne } \\
\text { albida }\end{array}$ & $\begin{array}{l}\text { Neptunia } \\
\text { oleracea }\end{array}$ & $\begin{array}{l}\text { Limnocharis } \\
\text { flava }\end{array}$ \\
\hline Lemna trisulca & & Limnophila indica & $\begin{array}{l}\text { Cryptocoryne } \\
\text { cordata }\end{array}$ & $\begin{array}{l}\text { Nymphoides } \\
\text { indica }\end{array}$ & $\begin{array}{l}\text { Myriophyllum } \\
\text { brasiliense }\end{array}$ \\
\hline Najas marina & & $\begin{array}{l}\text { Limnophila } \\
\text { sessiliflora }\end{array}$ & $\begin{array}{l}\text { Cryptocoryne } \\
\text { crispatula }\end{array}$ & Pistia stratiotes & $\begin{array}{l}\text { Myriophyllum } \\
\text { spicatum }\end{array}$ \\
\hline $\begin{array}{l}\text { Potamogeton } \\
\text { crispus }\end{array}$ & & $\begin{array}{l}\text { Monochoria } \\
\text { vaginalis }\end{array}$ & $\begin{array}{l}\text { Cryptocoryne } \\
\text { cruddasiana }\end{array}$ & Utricularia gibba & $\begin{array}{l}\text { Nelumbo } \\
\text { nucifera }\end{array}$ \\
\hline $\begin{array}{l}\text { Potamogeton } \\
\text { nodosus }\end{array}$ & & Mimulus orbicularis & $\begin{array}{l}\text { Eriocaulon } \\
\text { setaceum }\end{array}$ & & $\begin{array}{l}\text { Sagittaria } \\
\text { guayanensis }\end{array}$ \\
\hline Ruppia maritima & & $\begin{array}{l}\text { Nymphoides } \\
\text { aurantiaca }\end{array}$ & $\begin{array}{l}\text { Limnophila } \\
\text { heterophylla }\end{array}$ & & \\
\hline $\begin{array}{l}\text { Spirodela } \\
\text { polyrrhiza }\end{array}$ & & Najas graminea & Monochoria elata & & \\
\hline $\begin{array}{l}\text { Stuckenia } \\
\text { pectinata }\end{array}$ & & Ottelia alismoides & $\begin{array}{l}\text { Monochoria } \\
\text { hastata }\end{array}$ & & \\
\hline \multirow[t]{5}{*}{ Typha angustifolia } & & $\begin{array}{l}\text { Persicaria } \\
\text { attenuata subsp. } \\
\text { pulchra }\end{array}$ & $\begin{array}{l}\text { Myriophyllum } \\
\text { tetrandrum }\end{array}$ & & \\
\hline & & $\begin{array}{l}\text { Potamogeton } \\
\text { distinctus }\end{array}$ & $\begin{array}{l}\text { Myriophyllum } \\
\text { tuberculatum }\end{array}$ & & \\
\hline & & $\begin{array}{l}\text { Potamogeton } \\
\text { maackianus }\end{array}$ & Najas indica & & \\
\hline & & $\begin{array}{l}\text { Potamogeton } \\
\text { octandrus }\end{array}$ & Najas tenuis & & \\
\hline & & $\begin{array}{l}\text { Potamogeton } \\
\text { wrightii }\end{array}$ & $\begin{array}{l}\text { Nechamandra } \\
\text { alternifolia }\end{array}$ & & \\
\hline
\end{tabular}




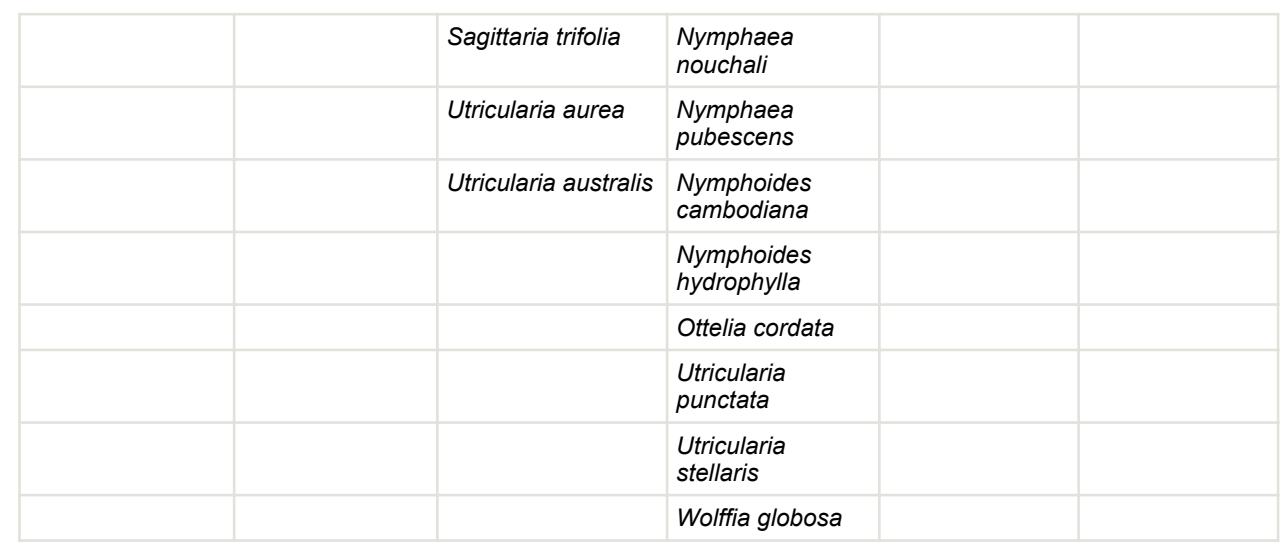

\section{Discussion}

Altogether, we identified 380 specimens, excluding 31 duplicates, belonging to 75 species of 41 genera from 23 plant families; the remaining 3 species belonging to 3 genera of 3 families were listed with no voucher specimens. Geographically the species distribution pattern can be classified into six categories: Widespread; Eurasian cool-temperate; Asia Australia; Tropical Asia; Pan-Tropics; and Invasive.

\section{Widespread}

This category is mostly congruent with 'Widespread Species' of Sculthorpe (1967).

\section{Eurasian cool-temperate}

The species clustered in this category are distributed mainly in cool-temperate region of Eurasia, thus limiting the distribution to a small portion of Southeast Asia (Myanmar: Shan state; Thailand: Northern states).

\section{Asia - Australia}

The species included in this category have a distribution in either Australia or East Asia or both, e.g., Australia: Mimulus orbicularis and Nymphoides aurantiaca; East Asia: Acorus gramineus, Blyxa japonica, Limnophila sessiliflora, Potamogeton distinctus, P. maackianus, P. wrightii, and Sagittaria trifolia; Australia and East Asia: Blyxa aubertii, B. echinosperma, Hydrocharis dubia, Limnophila indica, Monochoria vaginalis, Najas graminea, Ottelia alismoides, Persicaria attenuata subsp. pulchra, Potamogeton octandrus, Utricularia aurea, and $U$. australis. 


\section{Asian tropics}

This category comprises the largest number of species for the aquatic floras of Myanmar and Thailand. Some of the species included are endemic or narrowly distributed e.g., Cryptocoryne is endemic to tropical Asia. The monotypic genus Nechamandra occurs predominantly in India but also scattered in other parts of tropical Asia (also reported from one locality of Africa). Another monotypic genus Barclaya is among the tropical Asian endemics. The genera Aponogeton (Aponogetonaceae), Blyxa, Najas, (Hydrocharitaceae), Myriophyllum (Haloragaceae), Nymphoides (Menyanthaceae), Monochoria (Pontederiaceae), Utricularia (Lentibulariaceae) have their center of diversity in tropical Asia.

On a species level, the distribution is further classified into three groups: 1) Indo-China, 2) Southeast Asian, and 3) endemic to Myanmar and Thailand. The first group includes Cryptocoryne crispatula, Eriocaulon setaceum, Limnophila heterophylla, Monochoria hastata, Myriophyllum tetrandrum, M. tuberculatum, Najas indica, N. graminea, Nechamandra alternifolia, Nymphaea nouchali, N. pubescens, Nymphoides hydrophylla, and Utricularia stellaris (also known from Africa). The second group includes Barclaya longifolia, Cryptocoryne albida, C. cordata, Monochoria elata, and Ottelia cordata. The last group includes Blyxa quadricostata, Crinum thaianum, Cryptocoryne cruddasiana, and Utricularia punctata.

\section{Pan-tropics}

This category with eight species is mostly congruent with 'Pan-tropical Species' of Sculthorpe (1967).

\section{Invasive}

The aquatic floras of Myanmar and Thailand have well known invasive aquatic species including Elodea densa (Hydrocharitaceae) and Eichhornia crassipes (Pontederiaceae).

\section{Acknowledgements}

The authors would like to thank R. Pooma, S. Saengrit, N. Suphuntee (BKF), T. Koyama, Nb. Tanaka (MBK), J. Murata, T. Ohi-Toma, A. Shimizu (TI), Nr. Tanaka (TNS), A. Naiki (RYU), T. Sugawara (MAK), U. Htun Paw Oo, and U. Soe Win Hlaing (Myanmar). 


\section{References}

- Barkera WR, Jobson RW (2013) Mimulus orbicularis Benth. (Phrymaceae), a newly recorded aquatic from northern Queensland. Australasian Systematic Botany Society Newsletter 156: 11-12.

- Boyce PC (2012) Acoraceae. In: Santisuk T, Larsen K (Eds) Flora of Thailand. 11. The Forest Herbarium, Royal Forest Department, Bangkok, 322-325 pp.

- Boyce PC, Sookchaloem D, Hetterscheid WL, Gusman G, Jacobsen N, Idei T, Du NV (2012) Araceae. In: Santisuk T, Larsen K (Eds) Flora of Thailand. 11. The Forest Herbarium, Royal Forest Department, Bangkok, 101-321 pp.

- Chayamarit K (2005) Pontederiaceae. In: Santisuk T, Larsen K (Eds) Flora of Thailand. 9. The Forest Herbarium, Royal Forest Department, Bangkok, 51-57 pp.

- Cook CD (1996) Aquatic and Wetland Plants of India: A reference book and identification manual for the vascular plants found in permanent or seasonal fresh water in the subcontinent of India south of the Himalayas. Oxford University Press, Oxford, $385 \mathrm{pp}$.

- Guo YH, Haynes RR, Hellquist CB, Kaplan Z (2010) Potamogetonaceae. In: Wu ZY, Raven PH, Hong DY (Eds) Flora of China. 23. Science Press, Beijing, and Missouri Botanical Garden Press, St. Louis, $108-115 \mathrm{pp}$.

- Haston E, Richardson J, Stevens P, Chase M, Harris D (2009) The Linear Angiosperm Phylogeny Group (LAPG) III: a linear sequence of the families in APG III. Botanical Journal of the Linnean Society 161 (2): 128-131. DOI: 10.1111/j.1095-8339.2009.01000.x

- Haynes RR (2001a) Aponogetonaceae. In: Santisuk T, Larsen K (Eds) Flora of Thailand. The Forest Herbarium, Royal Forest Department, Bangkok, 359-360 pp.

- Haynes RR (2001b) Hydrocharitaceae. In: Santisuk T, Larsen K (Eds) Flora of Thailand. 7. The Forest Herbarium, Royal Forest Department, Bangkok, 365-382 pp.

- Haynes RR (2001c) Limnocharitaceae. In: Santisuk T, Larsen K (Eds) Flora of Thailand. 7. The Forest Herbarium, Royal Forest Department, Bangkok, 383-386 pp.

- Haynes RR (2001d) Alismataceae. In: Santisuk T, Larsen K (Eds) Flora of Thailand. 7. The Forest Herbarium, Royal Forest Department, Bangkok, 351-358 pp.

- Haynes RR (2001e) Cymodoceaceae. In: Santisuk T, Larsen K (Eds) Flora of Thailand. 7. The Forest Herbarium, Royal Forest Department, Bangkok, 361-364 pp.

- Haynes RR (2001f) Potamogetonaceae. In: Santisuk T, Larsen K (Eds) Flora of Thailand. 7. The Forest Herbarium, Royal Forest Department, Bangkok, 387-390 pp.

- Ho TN, Ornduff R (1995) Menyanthaceae. In: Wu ZY, Raven PH, Hong DY (Eds) Flora of China. 16. Science Press, Beijing, and Missouri Botanical Garden Press, St. Louis, 140-142 pp.

- Ito Y (2013a) Ruppiaceae. In: Santisuk T, Larsen K (Eds) Flora of Thailand. 12. The Forest Herbarium, Royal Forest Department, Bangkok.

- Ito Y (2013b) New records of aquatic monocots for the Flora of Thailand: Nechamandra alternifolia (Roxb. ex Wight) Thwaites (Hydrocharitaceae) and Potamogeton octandrus Poiret (Potamogetonaceae). Thai Forest Bulletin 41: 140-144.

- Ito Y, Tanaka N, Ohi-Toma T, Murata J (2009) New or noteworthy plant collection from Myanmar (3): Cardesia parnassifolia, Nechamandra alternifolia, Potamogeton maackianus and P. octandrus. Journal of Japanese Botany 84: 321-329.

- Kress WJ, Filipps RAD, Farr E, Kyi DY (2003) A Checklist of the Trees, Shrubs, Herbs, and Climbers of Union of Myanmar. Contributions from the Unites States National Herbarium, 45. National Museum of Natural History, Washington D.C., 590 pp.

- Landolt E (2001) Lemnaceae. In: Santisuk T, Larsen K (Eds) Flora of Thailand. 7. The Forest Herbarium, Royal Forest Department, Bangkok, 394-399 pp.

- Larsen K (2000) Callitrichaceae. In: Santisuk T, Larsen K (Eds) Flora of Thailand. 7. The Forest Herbarium, Royal Forest Department, Bangkok. 
- Sculthorpe CD (1967) Some aspects of the geography of aquatic vascular plants. In: Sculthorpe CD (Ed.) The Biology of Aquatic Vascular Plants. Edward Arnold Ltd., London, 365-413 pp.

- Simpson DA (2008) Typhaceae. In: Santisuk T, Larsen K (Eds) Flora of Thailand. 9. The Forest Herbarium, Royal Forest Department, Bangkok, 176-178 pp.

- Tippery N, Les D, Jacinto C. Regalado J, Averyanov L, Long VN, Raven P (2009) Transfer of Villarsia cambodiana to Nymphoides (Menyanthaceae). Systematic Botany 34 (4): 818-823. DOI: 10.1600/036364409790139673

- Van Dijk PP, Tordoff AW, Fellowes J, Lau M, Jinshuang M (2004) Indo-Burma. In: Mittermeier RA, Robles GP, Hoffmann M, Lamoreaux J, Fonseca GABd (Eds) Hotspots Revisited: Earth's Biologically Richest and Most Endangered Terrestrial Ecoregions. CEMEX, Agrupación Sierra Madre, 323-330 pp.

- Yamazaki T (1990) Scrophlariaceae. In: Smitinand T, Larsen K (Eds) Flora of Thailand. 5. The Forest Herbarium, Royal Forest Department, Bangkok, 139-238 pp. 